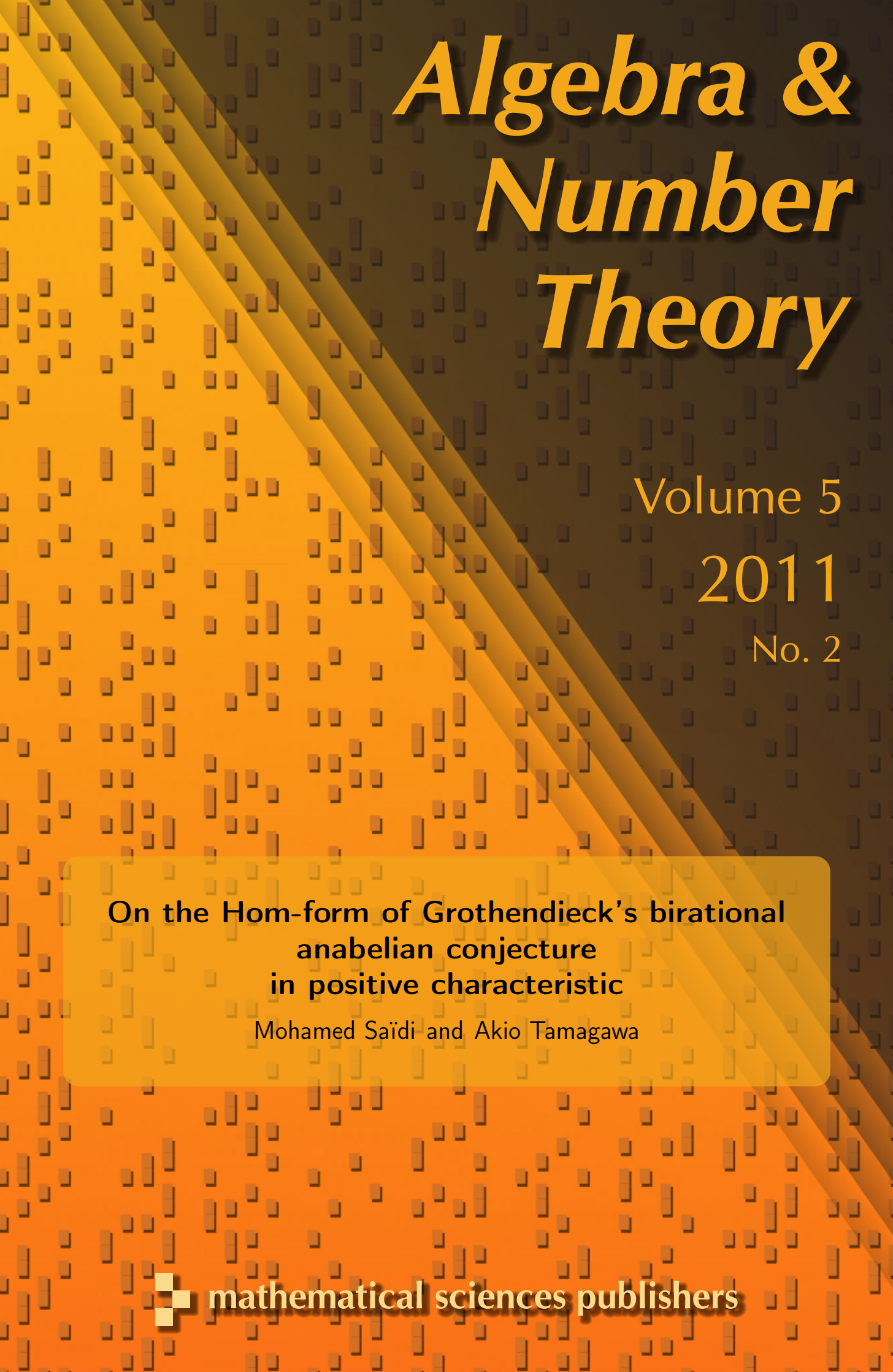

On the Hom-form of Grothendieck's birational anabelian conjecture in positive characteristic Mohamed Saïdi and Akio Tamagawa

\lrcorner 


\title{
On the Hom-form of Grothendieck's birational anabelian conjecture in positive characteristic
}

\author{
Mohamed Saïdi and Akio Tamagawa
}

We prove that a certain class of open homomorphisms between Galois groups of function fields of curves over finite fields arises from embeddings between the function fields.

1. Generalities on Galois groups of function fields of curves

2. Basic properties of homomorphisms between Galois groups

3. Rigid homomorphisms between Galois groups

4. Proper homomorphisms between Galois groups

5. Recovering the additive structure

\section{Introduction}

Let $K$ be an infinite field that is finitely generated over its prime field. Let $\bar{K}$ be an algebraic closure of $K$. We denote by $K^{\text {sep }}$ the separable closure, and by $K^{\text {perf }}$ the perfection, of $K$ in $\bar{K}$. Let $G_{K} \stackrel{\text { def }}{=} \operatorname{Gal}\left(K^{\text {sep }} / K\right)$ be the absolute Galois group of $K$. (Observe that $G_{K}=G_{K^{\text {perf }}}$.) The ultimate aim of Grothendieck's birational anabelian conjectures is to reconstruct the field structure of $K$ from the topological group structure of $G_{K}$. More precisely, these conjectures can be formulated as follows.

Saïdi was holding an EPSRC advanced research fellowship GR/R75861/02 during the preparation of this paper, and would very much like to thank EPSRC for its support.

MSC2010: primary 14G15; secondary 14H25, 14H30, $11 \mathrm{G} 20$.

Keywords: Grothendieck's birational anabelian conjecture, homomorphisms between Galois groups. 
Birational anabelian conjectures. There exists a group-theoretic recipe for recovering finitely generated infinite fields (or their perfections) from their absolute Galois groups $G_{K}$. In particular, if for such fields $K$ and $L$ one has $G_{K} \stackrel{\sim}{\rightarrow} G_{L}$, then $L^{\text {perf }} \stackrel{\sim}{\rightarrow} K^{\text {perf }}$. Moreover, given two such fields $K$ and $L$, one has the following.

Isom-form. Every isomorphism $\sigma: G_{K} \stackrel{\sim}{\rightarrow} G_{L}$ is defined by a field isomorphism $\bar{\gamma}: \bar{L} \stackrel{\sim}{\rightarrow} \bar{K}$. This isomorphism is unique if the characteristic is 0 , and unique up to Frobenius twists if the characteristic is positive. In particular, $\bar{\gamma}$ induces an isomorphism $L^{\text {perf }} \stackrel{\sim}{\rightarrow} K^{\text {perf }}$.

Hom-form. Every open homomorphism $\sigma: G_{K} \rightarrow G_{L}$ is defined by a field embedding $\bar{\gamma}: \bar{L} \hookrightarrow \bar{K}$. This embedding is unique if the characteristic is 0 , and unique up to Frobenius twists if the characteristic is positive. In particular, $\bar{\gamma}$ induces a field embedding $L^{\text {perf }} \hookrightarrow K^{\text {perf }}$.

Thus, the Hom-form is stronger than the Isom-form. The first results concerning these conjectures were obtained by Neukirch and Uchida in the case of global fields.

Theorem (Neukirch, Uchida). Let $K$ and $L$ be global fields. Then the natural map

$$
\operatorname{Isom}(L, K) \rightarrow \operatorname{Isom}\left(G_{K}, G_{L}\right) / \operatorname{Inn}\left(G_{L}\right)
$$

is a bijection.

More precisely, this is due to Neukirch [1969a; 1969b] and Uchida [1976] for number fields, and due to Uchida [1977] for function fields of curves over finite fields. Later, Pop generalized their results to the case of finitely generated fields of higher transcendence degree ([Pop 1994; 2002]; see also [Szamuely 2004] for a survey on Pop's results).

In characteristic 0 , Mochizuki proved the following relative version of the Homform of the birational conjectures.

Theorem [Mochizuki 1999]. Let $K$ and $L$ be two finitely generated, regular extensions of a field $k$. Assume that $k$ is a sub-p-adic field (that is, $k$ can be embedded in a finitely generated extension of $\mathbb{Q}_{p}$ ) for some prime number $p$. Then the natural map

$$
\operatorname{Hom}_{k}(L, K) \rightarrow \operatorname{Hom}_{G_{k}}^{\text {open }}\left(G_{K}, G_{L}\right) / \operatorname{Inn}\left(\operatorname{Ker}\left(G_{L} \rightarrow G_{k}\right)\right)
$$

is a bijection. Here, $\operatorname{Hom}_{k}$ denotes the set of $k$-embeddings, and $\operatorname{Hom}_{G_{k}}^{\text {open }}$ denotes the set of open $G_{k}$-homomorphisms.

However, almost nothing is known about the absolute version (that is, not relative with respect to a fixed base field $k$ ) of the Hom-form, except for Uchida's result [1981] for $K=\mathbb{Q}$ and $[L: \mathbb{Q}]<\infty$.

A major obstacle in proving the Hom-form of the birational anabelian conjectures is that one of the main common ingredients in the proofs of Neukirch, Uchida, 
and Pop, which is the so-called local theory (or Galois characterization of the decomposition subgroups), and which is used in order to establish a one-to-one correspondence between divisorial valuations, is not available in the case of open homomorphisms between Galois groups. More precisely, the main result of local theory available so far, Proposition 1.5, gives very little information on the image of the decomposition subgroups in this case, though one can still prove some partial results (Proposition 2.2, Lemmas 2.6 and 2.9). It seems quite difficult, for the moment, to establish a satisfactory local theory that is suitable to the Hom-form of the above conjecture. Also, the methods used in the proof of Mochizuki's theorem above are quite different, and do not rely on local theory. Instead, Mochizuki proves his result as an application of his fundamental anabelian result that relative open homomorphisms between arithmetic fundamental groups of curves over sub$p$-adic fields arise from morphisms between corresponding curves, the proof of which relies on $p$-adic Hodge theory. It is not clear how to adapt Mochizuki's method to the case of positive characteristics.

In this paper we investigate the Hom-form of the birational anabelian conjectures for function fields of curves over finite fields. For $i=1,2$, let $k_{i}$ be a finite field. Let $X_{i}$ be a proper, smooth, geometrically connected algebraic curve over $k_{i}$. Let $K_{i}$ be the function field of $X_{i}$ and fix an algebraic closure $\bar{K}_{i}$ of $K_{i}$. Let $K_{i}^{\text {sep }}$ and $K_{i}^{\text {perf }}$ be the separable closure and the perfection of $K_{i}$ in $\bar{K}_{i}$, and $\bar{k}_{i}$ the algebraic closure of $k_{i}$ in $\bar{K}_{i}$. Write $G_{i} \stackrel{\text { def }}{=} G_{K_{i}} \stackrel{\text { def }}{=} \operatorname{Gal}\left(K_{i}^{\text {sep }} / K_{i}\right)$ for the absolute Galois group of $K_{i}$, and $G_{k_{i}} \stackrel{\text { def }}{=} \mathrm{Gal}\left(\bar{k}_{i} / k_{i}\right)$ for the absolute Galois group of $k_{i}$. We have the natural exact sequence of profinite groups

$$
1 \rightarrow \bar{G}_{i} \rightarrow G_{i} \stackrel{\mathrm{pr}_{i}}{\rightarrow} G_{k_{i}} \rightarrow 1
$$

where $\bar{G}_{i}$ is the absolute Galois $\operatorname{group} \operatorname{Gal}\left(K_{i}^{\mathrm{sep}} / K_{i} \bar{k}_{i}\right)$ of $K_{i} \bar{k}_{i}$, and $\mathrm{pr}_{i}$ is the canonical projection.

Further, let $p_{i}$ be the characteristic of $k_{i}$, and let $\bar{G}_{i}^{p_{i}^{\prime}}$ be the maximal prime-to$p_{i}$ quotient of $\bar{G}_{i}$. The push-forward of this sequence with respect to the natural surjection $\bar{G}_{i} \rightarrow \bar{G}_{i}^{p_{i}^{\prime}}$ gives rise to the natural exact sequence

$$
1 \rightarrow \bar{G}_{i}^{p_{i}^{\prime}} \rightarrow G_{i}^{\left(p_{i}^{\prime}\right) \stackrel{\mathrm{pr}_{i}}{\rightarrow}} G_{k_{i}} \rightarrow 1 .
$$

Set $\mathfrak{G}_{i} \stackrel{\text { def }}{=} G_{i}, i=1,2$ (which we call the profinite case) or $\mathfrak{G}_{i} \stackrel{\text { def }}{=} G_{i}^{\left(p_{i}^{\prime}\right)}, i=1,2$ (the prime-to-characteristic case). We investigate two classes of continuous, open homomorphisms — rigid and proper homomorphisms — between $\mathfrak{G}_{1}$ and $\mathfrak{G}_{2}$.

First, we investigate a class of continuous, open homomorphisms $\sigma: \mathfrak{G}_{1} \rightarrow \mathfrak{G}_{2}$, which we call rigid. More precisely, we say that $\sigma$ is strictly rigid if the image of each decomposition subgroup of $\mathfrak{G}_{1}$ coincides with a decomposition subgroup of $\mathfrak{G}_{2}$, and we say that $\sigma$ is rigid if there exist open subgroups $\mathfrak{H}_{1} \subset \mathfrak{G}_{1}, \mathfrak{H}_{2} \subset \mathfrak{G}_{2}$, such 
that $\sigma\left(\mathfrak{H}_{1}\right) \subset \mathfrak{H}_{2}$ and that $\mathfrak{H}_{1} \stackrel{\sigma}{\rightarrow} \mathfrak{H}_{2}$ is strictly rigid. Thus, isomorphisms between $\mathfrak{G}_{1}$ and $\mathfrak{G}_{2}$ are (strictly) rigid by the main result of local theory for the Isom-form. Let $\operatorname{Hom}\left(\mathfrak{G}_{1}, \mathfrak{G}_{2}\right)^{\text {rig }}$ be the set of rigid homomorphisms between $\mathfrak{G}_{1}$ and $\mathfrak{G}_{2}$.

We say that a homomorphism $\gamma: K_{2} \rightarrow K_{1}$ of fields (which defines an extension $K_{1} / K_{2}$ of fields) is admissible if the extension $K_{1} / K_{2}$ appears in the extensions of $K_{2}$ corresponding to the open subgroups of $\mathfrak{G}_{2}$. An equivalent condition in the profinite case is that the extension $K_{1} / K_{2}$ is finite separable; and in the primeto-characteristic case, that the extension $K_{1} / K_{2}$ is finite separable and the Galois closure of the extension $K_{1} \bar{k}_{1} / K_{2} \bar{k}_{2}$ is of degree prime to $p \stackrel{\text { def }}{=} p_{1}=p_{2}$. We define $\operatorname{Hom}\left(K_{2}, K_{1}\right)^{\text {adm }} \subset \operatorname{Hom}\left(K_{2}, K_{1}\right)$ to be the set of admissible homomorphisms $K_{2} \rightarrow K_{1}$.

Now, our first main result is the following (see Theorem 3.4).

Theorem A. The natural map $\operatorname{Hom}\left(K_{2}, K_{1}\right) \rightarrow \operatorname{Hom}\left(\mathfrak{G}_{1}, \mathfrak{G}_{2}\right) / \operatorname{Inn}\left(\mathfrak{G}_{2}\right)$ induces a bijection

$$
\operatorname{Hom}\left(K_{2}, K_{1}\right)^{\mathrm{adm}} \stackrel{\sim}{\rightarrow} \operatorname{Hom}\left(\mathfrak{G}_{1}, \mathfrak{G}_{2}\right)^{\mathrm{rig}} / \operatorname{Inn}\left(\mathfrak{G}_{2}\right) .
$$

Our method of proving Theorem A is as follows. First, we prove, using a certain weight argument based on the Weil conjecture for curves, that a strictly rigid homomorphism $\sigma: \mathfrak{G}_{1} \rightarrow \mathfrak{G}_{2}$ induces a bijection $\Sigma_{X_{1}} \stackrel{\sim}{\rightarrow} \Sigma_{X_{2}}$ between the set of closed points of $X_{1}$ and $X_{2}$ (see Lemma 3.8). With this we can reduce the Homform in this case to the Isom-form, which has been established in [Uchida 1977] (profinite case) and [Saïdi and Tamagawa 2009] (prime-to-characteristic case).

Next we consider a class of continuous, open homomorphisms $\sigma: \mathfrak{G}_{1} \rightarrow \mathfrak{G}_{2}$, which we call proper. These are homomorphisms with the property that the image of each decomposition subgroup of $\mathfrak{G}_{1}$ coincides with an open subgroup of a decomposition subgroup of $\mathfrak{G}_{2}$, such that each decomposition subgroup of $\mathfrak{G}_{2}$ contains images of only finitely many conjugacy classes of decomposition subgroups of $\mathfrak{G}_{1}$. We also consider a certain rigidity condition, which we call inertiarigidity, on the various identifications between the modules of the roots of unity (Definition 4.5). Unfortunately, we are not able to prove that this condition automatically holds for proper homomorphisms. Let $\operatorname{Hom}\left(\mathfrak{G}_{1}, \mathfrak{G}_{2}\right)^{\text {pr,inrig }}$ be the set of proper and inertia-rigid homomorphisms between $\mathfrak{G}_{1}$ and $\mathfrak{G}_{2}$. Our second main result is the following (see Theorem 4.8).

Theorem B. The natural map $\operatorname{Hom}\left(K_{2}, K_{1}\right) \rightarrow \operatorname{Hom}\left(\mathfrak{G}_{1}, \mathfrak{G}_{2}\right) / \operatorname{Inn}\left(\mathfrak{G}_{2}\right)$ induces a bijection

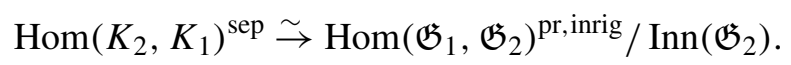

Here, we define $\operatorname{Hom}\left(K_{2}, K_{1}\right)^{\mathrm{sep}} \subset \operatorname{Hom}\left(K_{2}, K_{1}\right)$ to be the set of separable homomorphisms $K_{2} \rightarrow K_{1}$.

To prove Theorem B, we first show, using a weight argument, that a homomorphism $\sigma: \mathfrak{G}_{1} \rightarrow \mathfrak{G}_{2}$ as above induces a surjective map $\Sigma_{X_{1}} \rightarrow \Sigma_{X_{2}}$ between the sets 
of closed points of $X_{1}$ and $X_{2}$, which has finite fibers (Lemma 2.9). Second, using Kummer theory, we reconstruct functorially an embedding $K_{2}^{\times} \hookrightarrow\left(K_{1}^{\text {perf }}\right)^{\times}$between multiplicative groups (Lemma 4.13). Finally, we show that this embedding $K_{2}^{\times} \hookrightarrow\left(K_{1}^{\text {perf }}\right)^{\times}$is additive.

Recovering the additive structure is one of the main steps in the proof. This problem was treated by Uchida in the case of a bijective identification $K_{2}^{\times} \stackrel{\sim}{\rightarrow} K_{1}^{\times}$ between multiplicative groups, which is order-preserving and value-preserving. In fact, one needs only to restore the additivity between constants. For this one has to show identities of the form $\gamma\left(f_{2}+1\right)=\gamma\left(f_{2}\right)+1$ for some specific nonconstant function $f_{2} \in K_{2}$. Uchida succeeded in his case by choosing $f_{2}$ to be a function with a minimal pole divisor (he called such a function a minimal element.) His argument fails in the case of an embedding between multiplicative groups that is not surjective, because the image of a minimal element is not necessarily minimal in this case. Roughly speaking, we extend his arguments by using, instead, a function that has a unique pole. This one-pole argument turns out to be very efficient, and leads to the recovery of the additive structure under quite general assumptions (Proposition 5.3).

Although rigid homomorphisms are a special case of proper homomorphisms, we choose to treat them separately for several reasons. First, the important condition of inertia-rigidity is automatically satisfied in the case of rigid homomorphisms (Remark 4.9(i)). Second, in the case of (strictly) rigid homomorphisms we can reduce directly to the Isom-form, the proof of which can be based on class field theory. This is not possible for proper homomorphisms, in general. In fact, in the case of proper homomorphisms, class field theory reconstructs only the norm map between the multiplicative groups of function fields.

This paper is organized as follows. In Section 1, we review well-known facts concerning Galois theory of function fields of curves over finite fields, including the main results of local theory. In Section 2, we investigate some basic properties of homomorphisms between absolute Galois groups of function fields of curves over finite fields, as well as homomorphisms between decomposition subgroups. In Section 3, we investigate rigid homomorphisms between (geometrically primeto-characteristic quotients of) absolute Galois groups, and prove Theorem A. In Section 4, we investigate proper homomorphisms between (geometrically primeto-characteristic quotients of) absolute Galois groups, and prove Theorem B. In Section 5, we investigate the problem of recovering the additive structure of function fields. Using the above one-pole argument, we prove Proposition 5.3, which is used in the proof of Theorem B in Section 4.

We hope very much that this paper is a first step towards proving the Hom-form of Grothendieck's anabelian conjecture concerning arithmetic fundamental groups 
of hyperbolic curves over finite fields, whose Isom-form was proven by Tamagawa [1997] for affine curves and Mochizuki [2007] for proper curves.

\section{Generalities on Galois groups of function fields of curves}

1A. Notations on profinite groups and fields. Let $\mathscr{C}$ be a full class of finite groups ( $\mathscr{C}$ is closed under taking subgroups, quotients, finite products, and extensions). For a profinite group $H$, denote by $H^{\mathscr{C}}$ the maximal pro- $\mathscr{C}$ quotient of $H$, and set $H^{(\mathscr{C})} \stackrel{\text { def }}{=} H / \operatorname{Ker}\left(\bar{H} \rightarrow \bar{H}^{\mathscr{C}}\right)$, where $\bar{H}$ is a closed normal subgroup of $H$. Note that $H^{(\mathscr{C})}$ coincides with $H^{\mathscr{C}}$ if and only if the quotient $A \stackrel{\text { def }}{=} H / \bar{H}$ is a pro- $\mathscr{C}$ group. By definition, we have the commutative diagram

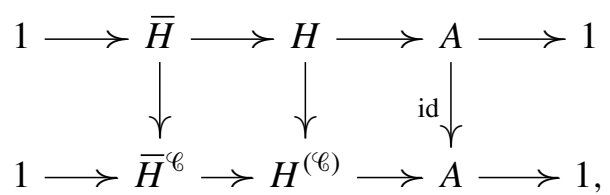

where the rows are exact and the columns are surjective.

If $l$ is a prime number, we write $H^{l}$ and $H^{(l)}$ instead of $H^{\mathscr{C}}$ and $H^{(\mathscr{C})}$ when $\mathscr{C}$ is the class of finite $l$-groups, and we write $H^{l^{\prime}}$ and $H^{\left(l^{\prime}\right)}$ when $\mathscr{C}$ is the class of finite $l^{\prime}$-groups (finite groups of order prime to $l$ ).

For a profinite group $H$, we write $H^{\text {ab }}$ for the maximal abelian quotient of $H$; $\operatorname{Sub}(H)$ for the set of closed subgroups of $H$; $\operatorname{Aut}(H)$ for the group of (continuous) automorphisms of $H$; and $\operatorname{Inn}(H)$ for the group of inner automorphisms of $H$.

For a profinite group $H$ and a prime number $l$, denote by $\operatorname{cd}(H)$ and $\operatorname{cd}_{l}(H)$ the cohomological and $l$-cohomological dimensions of $H$. It is well-known that if $\operatorname{cd}(H)<\infty$, then $H$ is torsion-free.

Let $\kappa$ be a field and $\kappa^{\text {sep }}$ a separable closure of $\kappa$. Denote the absolute Galois group $\operatorname{Gal}\left(\kappa^{\text {sep }} / \kappa\right)$ by $G_{\kappa}$. We write

$$
M_{\kappa^{\text {sep }}} \stackrel{\text { def }}{=} \operatorname{Hom}\left(\mathbb{Q} / \mathbb{Z},\left(\kappa^{\text {sep }}\right)^{\times}\right) .
$$

Thus, $M_{\kappa^{\text {sep }}}$ is a free $\hat{\mathbb{Z}}^{\dagger}$-module of rank one, where $\hat{\mathbb{Z}}^{\dagger}$ is defined as $\hat{\mathbb{Z}}$ if $\operatorname{char} \kappa=0$ and as $\hat{\mathbb{Z}}^{p^{\prime}}$ if char $\kappa=p>0$. Further, $M_{\kappa}$ sep has a natural structure of $G_{\kappa}$-module, which is isomorphic to the Tate twist $\hat{\mathbb{Z}}^{\dagger}(1)$; that is, $G_{\kappa}$ acts on $M_{\kappa}$ sep via the cyclotomic character $\chi_{\kappa}: G_{\kappa} \rightarrow\left(\hat{\mathbb{Z}}^{\dagger}\right)^{\times}$.

1B. Galois groups of local fields of positive characteristic. Let $p$ be a prime number. Let $L$ be a local field of characteristic $p$, that is, a complete discrete valuation field of equal characteristic $p$, with finite residue field $\ell$. We denote the ring of integers of $L$ by $O_{L}$. Also, fix a separable closure $L^{\text {sep }}$ of $L$. We shall denote the residue field of $L^{\text {sep }}$ by $\bar{\ell}$, since it is an algebraic closure of $\ell$. Note that $\ell$ and $\bar{\ell}$ can also be regarded naturally as subfields of $L$ and $L^{\text {sep }}$, respectively. Write 
$D \stackrel{\text { def }}{=} \operatorname{Gal}\left(L^{\mathrm{sep}} / L\right)$ for the corresponding absolute Galois group of $L$, and define the inertia group of $L$ by

$$
I \stackrel{\text { def }}{=}\{\gamma \in D \mid \gamma \text { acts trivially on } \bar{\ell}\} .
$$

We have a canonical exact sequence

$$
1 \rightarrow I \rightarrow D \rightarrow G_{\ell} \stackrel{\text { def }}{=} \operatorname{Gal}(\bar{\ell} / \ell) \rightarrow 1
$$

and, for a full class $\mathscr{C}$ of finite groups, we get a canonical exact sequence

$$
1 \rightarrow I^{\mathscr{C}} \rightarrow D^{(\mathscr{C})} \rightarrow G_{\ell} \rightarrow 1
$$

The inertia subgroup $I$ possesses a unique $p$-Sylow subgroup $I^{\mathrm{w}}$. The quotient $I^{\mathrm{t}} \stackrel{\text { def }}{=} I / I^{\mathrm{w}}$ is isomorphic to $\hat{\mathbb{Z}}^{p^{\prime}}$, and is naturally identified with the Galois group $\operatorname{Gal}\left(L^{\mathrm{t}} / L^{\mathrm{ur}}\right)$, where $L^{\mathrm{t}}$ and $L^{\mathrm{ur}}$ are the maximal tamely ramified and maximal unramified extensions of $L$ contained in $L^{\text {sep }}$. We have a natural exact sequence

$$
1 \rightarrow I^{\mathrm{t}} \rightarrow D^{\mathrm{t}} \rightarrow G_{\ell} \rightarrow 1
$$

where $D^{\mathrm{t}} \stackrel{\text { def }}{=} \operatorname{Gal}\left(L^{\mathrm{t}} / L\right)$. (Observe that $I^{\mathrm{t}}=I^{p^{\prime}}$ and $D^{\mathrm{t}}=D^{\left(p^{\prime}\right)}$.) In particular, $I^{\mathrm{t}}$ has a natural structure of $G_{\ell}$-module. Further, there exists a natural identification $I^{\mathrm{t}} \stackrel{\sim}{\rightarrow} M_{\bar{\ell}}$ of $G_{\ell}$-modules. These follow from well-known facts in ramification theory. See [Serre 1968, chapitre IV] for more details.

Let $l$ be a prime number. Denote by $D_{l}$ an $l$-Sylow subgroup of $D$. Then the intersection $I_{l} \stackrel{\text { def }}{=} I \cap D_{l}$ is an $l$-Sylow subgroup of $I$. Thus, $I_{p}=I^{\mathrm{w}}$ and, for $l \neq p$, $I_{l}$ is isomorphic to $\mathbb{Z}_{l}$. The image $G_{\ell, l}$ of $D_{l}$ in $G_{\ell}$ is the unique $l$-Sylow subgroup

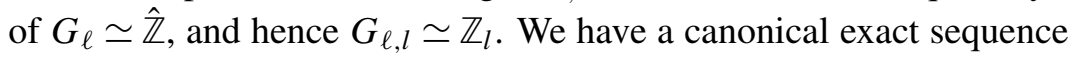

$$
1 \rightarrow I_{l} \rightarrow D_{l} \rightarrow G_{\ell, l} \rightarrow 1
$$

In particular, $I_{l}$ has a natural structure of $G_{\ell, l}$-module, and, if $l \neq p$, there exists a natural identification $I_{l} \stackrel{\sim}{\rightarrow} M_{\bar{\ell}, l}$ of $G_{\ell, l}$-modules, where $M_{\bar{\ell}, l}$ stands for the $l$-Sylow subgroup of the profinite abelian group $M_{\bar{\ell}}$.

It is well-known that $\operatorname{cd}_{l}(D)=\operatorname{cd}\left(D_{l}\right)=2$ for any prime number $l \neq p$, and that $\operatorname{cd}_{p}(D)=\operatorname{cd}\left(D_{p}\right)=1$. Thus, $\operatorname{cd}(D)=2<\infty$. In particular, $D$ is torsion-free.

Proposition 1.1. Let $\mathfrak{D}$ be a quotient of $D$, let $\mathfrak{I}$ be the image of $I$ in $\mathfrak{D}$, and let $\mathfrak{G}_{\ell} \stackrel{\text { def }}{=} \mathfrak{D} / \mathfrak{I}$. For each prime number $l$, let $\mathfrak{D}_{l}, \mathfrak{I}_{l}$ and $\mathfrak{G}_{\ell, l}$ be the images of $D_{l}, I_{l}$ and $G_{\ell, l}$ in $\mathfrak{D}, \mathfrak{I}$ and $\mathfrak{G}_{\ell}$, respectively, which are each $l$-Sylow subgroups of $\mathfrak{D}, \mathfrak{I}$ and $\mathfrak{G}_{\ell}$, respectively. Let $l$ be a prime number $\neq p$.

(i) One of the following cases occurs.

Case 0: $\quad \operatorname{cd}_{l}(\mathfrak{D})=0, \mathfrak{D}_{l}=\{1\}, \mathfrak{I}_{l}=\{1\}$, and $\mathfrak{G}_{\ell, l}=\{1\}$.

Case 1: $\operatorname{cd}_{l}(\mathfrak{D})=1, \mathfrak{D}_{l} \simeq G_{\ell}, \mathfrak{I}_{l}=\{1\}$, and $\mathfrak{G}_{\ell, l} \simeq G_{\ell}$.

Case 2: $\operatorname{cd}_{l}(\mathfrak{D})=2, \mathfrak{D}_{l} \simeq D_{l}, \mathfrak{I}_{l} \simeq I_{l}$, and $\mathfrak{G}_{\ell, l} \simeq G_{\ell}$. 
Case $\infty: \operatorname{cd}_{l}(\mathfrak{D})=\infty$, and $\mathfrak{I}_{l}$ is a finite group.

(ii) Assume that Case 2 occurs. Let $\mathfrak{D}^{\prime}$ be an open subgroup of $\mathfrak{D}, L^{\prime}$ the (finite, separable) extension of $L$ corresponding to $\mathfrak{D}^{\prime} \subset \mathfrak{D}$, and $D^{\prime}$ the inverse image of $\mathfrak{D}^{\prime}$ in $D$. (Thus, $D^{\prime}=G_{L^{\prime}}$.) Then, for each finite l-primary $\mathfrak{D}^{\prime}$-module $M$ and each $k \geq 0$, one has $H^{k}\left(\mathfrak{D}^{\prime}, M\right) \stackrel{\sim}{\rightarrow} H^{k}\left(D^{\prime}, M\right)$.

Proof. (i) Since $\mathfrak{I}_{l}$ is a quotient of $I_{l} \simeq \mathbb{Z}_{l}$, one of the following occurs: (a) $\mathfrak{I}_{l}=\{1\}$, (b) $\mathfrak{I}_{l} \simeq \mathbb{Z} / l^{m} \mathbb{Z}$ for an integer $m>0$, and (c) $\mathfrak{I}_{l} \simeq \mathbb{Z}_{l}$. If (a), $\mathfrak{D}_{l}$ is a quotient of $D_{l} / I_{l}=G_{\ell} \simeq \mathbb{Z}_{l}$. Thus, it is easy to see that one of Cases 0,1 , or $\infty$ occurs. If (b), Case $\infty$ occurs. If (c), we have $\mathfrak{G}_{\ell, l} \simeq G_{\ell, l}$. This follows from the fact that $I_{l}$ is isomorphic to $M_{\bar{\ell}, l}$ on which $G_{\ell, l}$ acts via the $l$-adic cyclotomic character, and that the $l$-adic cyclotomic charter $\chi_{l}: G_{\ell, l} \rightarrow \mathbb{Z}_{l}^{\times}$is injective. Thus, it is easy to see that Case 2 occurs.

(ii) Replacing $L$ by $L^{\prime}$, we may assume that $L^{\prime}=L$. (Observe that Case 2 occurs also for the quotient $G_{L^{\prime}}=D^{\prime} \rightarrow \mathfrak{D}^{\prime}$.)

Denote by $N$ the kernel of the surjection $D \rightarrow \mathfrak{D}$. By the assumption that Case 2 occurs, $D_{l}$ is injectively mapped into $\mathfrak{D}$, and hence $D_{l} \cap N$, which is an $l$-Sylow subgroup of $N$, is trivial. Since $N$ is of order prime to $l$, we have $H^{k}(D, M)=$ $H^{k}\left(\mathfrak{D}, H^{0}(N, M)\right)=H^{k}(\mathfrak{D}, M)$, as desired.

1C. Galois groups of function fields of curves. Let $k$ be a finite field of characteristic $p>0$. Let $X$ be a proper, smooth, geometrically connected curve over $k$. Let $K=K_{X}$ be the function field of $X$ and fix an algebraic closure $\bar{K}$ of $K$. Write $K^{\text {sep }}$ and $\bar{k}=k^{\text {sep }}$ for the separable closures of $K$ and $k$ in $\bar{K}$. Write $G=G_{K} \stackrel{\text { def }}{=} \operatorname{Gal}\left(K^{\text {sep }} / K\right)$ and $G_{k} \stackrel{\text { def }}{=} \operatorname{Gal}(\bar{k} / k)$ for the absolute Galois groups of $K$ and $k$, respectively. We have the exact sequence of profinite groups

$$
1 \rightarrow \bar{G} \rightarrow G \stackrel{\text { pr }}{\rightarrow} G_{k} \rightarrow 1,
$$

where $\bar{G}$ is the absolute Galois group $G_{K \bar{k}}=\operatorname{Gal}\left(K^{\mathrm{sep}} / K \bar{k}\right)$ of $K \bar{k}$, and pr is the canonical projection. Here, it is well-known that the right term $G_{k}$ is a profinite free group of rank 1 that is (topologically) generated by the Frobenius element, while the left term $\bar{G}$ is a profinite free group of countably infinite rank [Pop 1995; Harbater 1995]. However, the structure of the extension (1.1) itself is not understood well. From (1.1) above, we also obtain the exact sequence

$$
1 \rightarrow \bar{G}^{\mathscr{C}} \rightarrow G^{(\mathscr{C})} \stackrel{\mathrm{pr}}{\rightarrow} G_{k} \rightarrow 1
$$

for each full class $\mathscr{C}$ of finite groups.

In the rest of this section, let $N$ be a closed normal subgroup of $G$ and set $\mathfrak{G} \stackrel{\text { def }}{=} G / N$. Let $\tilde{K}$ denote the Galois extension of $K$ corresponding to $N$, that is, $\tilde{K} \stackrel{\text { def }}{=}\left(K^{\text {sep }}\right)^{N}$. Let $\overline{\mathfrak{G}}$ be the image of $\bar{G}$ in $\mathfrak{G}$, and set $\mathfrak{G}_{k} \stackrel{\text { def }}{=} \mathfrak{G} / \overline{\mathfrak{G}}$, which is a quotient of $G_{k}$. 
For a scheme $T$, denote by $\Sigma_{T}$ the set of closed points of $T$. Write $\tilde{\tilde{X}}$ for the integral closure of $X$ in $K^{\text {sep }}$. The absolute Galois group $G$ acts naturally on the set $\Sigma_{\tilde{\tilde{X}}}$, and the quotient $\Sigma_{\tilde{\tilde{X}}} / G$ is naturally identified with $\Sigma_{X}$. For a point $\tilde{\tilde{x}} \in \Sigma_{\tilde{\tilde{X}}}$, with residue field $k(\tilde{\tilde{x}})$ (which is naturally identified with $\bar{k}$ ), we define its decomposition group $D_{\tilde{\tilde{x}}}$ and inertia group $I_{\tilde{\tilde{x}}}$ by

$$
D_{\tilde{\tilde{x}}} \stackrel{\text { def }}{=}\{\gamma \in G \mid \gamma(\tilde{\tilde{x}})=\tilde{\tilde{x}}\}
$$

and

$$
I_{\tilde{\tilde{x}}} \stackrel{\text { def }}{=}\left\{\gamma \in D_{\tilde{\tilde{x}}} \mid \gamma \text { acts trivially on } k(\tilde{\tilde{x}})\right\},
$$

respectively. We have a canonical exact sequence

$$
1 \rightarrow I_{\tilde{\tilde{x}}} \rightarrow D_{\tilde{\tilde{x}}} \rightarrow G_{k(x)} \rightarrow 1,
$$

where $x$ stands for the image of $\tilde{\tilde{x}}$ in $\Sigma_{X}$.

More generally, write $\tilde{X}$ for the integral closure of $X$ in $\tilde{K}$. The Galois group $\mathfrak{G}$ acts naturally on the set $\Sigma_{\tilde{X}}$, and the quotient $\Sigma_{\tilde{X}} / \mathfrak{G}$ is naturally identified with $\Sigma_{X}$. For a point $\tilde{x} \in \Sigma_{\tilde{X}}$, with residue field $k(\tilde{x})$ (which is naturally identified with a subfield of $\bar{k}$ ), we define its decomposition group $\mathfrak{D}_{\tilde{x}}$ and inertia group $\mathfrak{I}_{\tilde{x}}$ by

$$
\mathfrak{D}_{\tilde{x}} \stackrel{\text { def }}{=}\{\gamma \in \mathfrak{G} \mid \gamma(\tilde{x})=\tilde{x}\}
$$

and

$$
\mathfrak{I}_{\tilde{x}} \stackrel{\text { def }}{=}\left\{\gamma \in \mathfrak{D}_{\tilde{x}} \mid \gamma \text { acts trivially on } k(\tilde{x})\right\},
$$

respectively. (For any $g \in \mathfrak{G}$, one has $\mathfrak{D}_{g \tilde{x}}=g \mathfrak{D}_{\tilde{x}} g^{-1}$ and $\mathfrak{I}_{g \tilde{x}}=g \mathfrak{I}_{\tilde{x}} g^{-1}$.) Set $\mathfrak{G}_{k(x)} \stackrel{\text { def }}{=} \mathfrak{D}_{\tilde{x}} / \mathfrak{I}_{\tilde{x}}$. Thus, if we take a point $\tilde{\tilde{x}} \in \Sigma_{\tilde{\tilde{X}}}$ above $\tilde{x} \in \Sigma_{\tilde{X}}$, then $\mathfrak{D}_{\tilde{x}}, \mathfrak{I}_{\tilde{x}}$, and $\mathfrak{G}_{k(x)}$ are quotients of $D_{\tilde{\tilde{x}}}, I_{\tilde{\tilde{x}}}$ and $G_{k(x)}$, respectively, where $x$ stands for the image of $\tilde{\tilde{x}}$ in $\Sigma_{X}$. We have a canonical exact sequence

$$
1 \rightarrow \mathfrak{I}_{\tilde{x}} \rightarrow \mathfrak{D}_{\tilde{x}} \rightarrow \mathfrak{G}_{k(x)} \rightarrow 1 .
$$

For each closed subgroup $\mathfrak{H} \subset \mathfrak{G}$, denote by $\tilde{x}_{\mathfrak{H}}$ the image of $\tilde{x}$ in $X_{\mathfrak{H}}$. Define

$$
\tilde{K}_{\tilde{x}} \stackrel{\text { def }}{=} \bigcup_{\mathfrak{H} \subset \mathfrak{G}}\left(K_{\mathfrak{H}}\right)_{\tilde{x}_{\mathfrak{H}}},
$$

where $\mathfrak{H}$ runs over all open subgroups of $\mathfrak{G}$, and $\left(K_{\mathfrak{H}}\right) \tilde{x}_{\mathfrak{H}}$ means the $\tilde{x}_{\mathfrak{H}}$-adic completion of $K_{\mathfrak{H}} \stackrel{\text { def }}{=}(\tilde{K})^{\mathfrak{H}}$. Then the Galois group $\operatorname{Gal}\left(\tilde{K}_{\tilde{x}} / K_{x}\right)$ is naturally identified with $\mathfrak{D}_{\tilde{x}}$, where $x \stackrel{\text { def }}{=} \tilde{x}_{\mathfrak{G}} \in \Sigma_{X}$.

In the rest of this subsection, we fix a prime number $l \neq p$, and make two assumptions: (1) $N^{l}=N$, or, equivalently, $\tilde{K}$ admits no $l$-cyclic extension; and (2) $\tilde{K}$ contains a primitive $l$-th root of unity.

Remark 1.2. Let $\mathscr{C}$ be a full class of finite groups.

(i) If $\mathbb{F}_{l} \in \mathscr{C}$, then the quotient $G^{(\mathscr{C})}$ of $G$ satisfies these two assumptions. 
(ii) If $\mathbb{F}_{l} \in \mathscr{C}$ and $\operatorname{Gal}\left(K\left(\zeta_{l}\right) / K\right) \in \mathscr{C}$, then the quotient $G^{\mathscr{C}}$ of $G$ satisfies these two assumptions.

Lemma 1.3. Let $\tilde{x} \in \Sigma_{\tilde{X}}$ and take $\tilde{\tilde{x}} \in \Sigma_{\tilde{\tilde{X}}}$ above $\tilde{x}$. Let $D_{\tilde{\tilde{x}}, l}$ be an l-Sylow subgroup of $D_{\tilde{\tilde{x}}}$ and $\mathfrak{D}_{\tilde{x}, l}$ the image of $D_{\tilde{\tilde{x}}, l}$ under the natural surjection $D_{\tilde{\tilde{x}}} \rightarrow \mathfrak{D}_{\tilde{x}}$, which is an l-Sylow subgroup of $\mathfrak{D}_{\tilde{x}}$. Then the natural surjection $D_{\tilde{\tilde{x}}, l} \rightarrow \mathfrak{D}_{\tilde{x}, l}$ is an isomorphism.

Proof. Take $t \in K$ such that $t$ is a uniformizer at $x \stackrel{\text { def }}{=} \tilde{x}_{\mathfrak{G}} \in \Sigma_{X}$. Then by the two assumptions (and by Kummer theory), any $l^{n}$-th root $t^{1 / l^{n}}$ of $t$ is contained in $\tilde{K}$. From this, it follows that $I_{\tilde{x}, l} \stackrel{\text { def }}{=} D_{\tilde{\tilde{x}}, l} \cap I_{\tilde{\tilde{x}}}$ is injectively mapped into $\mathfrak{D}_{\tilde{x}}$. Now, applying Proposition 1.1(i) to the quotient $G_{K_{x}}=D_{\tilde{\tilde{x}}} \rightarrow \mathfrak{D}_{\tilde{x}}$, we conclude that only Case 2 from that proposition can occur, as desired.

Lemma 1.4. Let $\mathfrak{G}^{\prime}$ be an open subgroup of $\mathfrak{G}, K^{\prime}$ the (finite, separable) extension of $K$ corresponding to $\mathfrak{G}^{\prime} \subset \mathfrak{G}$, and $G^{\prime}$ the inverse image of $\mathfrak{G}^{\prime}$ in $G$. (Thus, $G^{\prime}=G_{K^{\prime}}$.) Then, for each finite l-primary $\mathfrak{G}^{\prime}$-module $M$ and each $k \geq 0$, one has $H^{k}\left(\mathfrak{G}^{\prime}, M\right) \stackrel{\sim}{\rightarrow} H^{k}\left(G^{\prime}, M\right)$.

Proof. Replacing $K$ by $K^{\prime}$, we may assume that $K^{\prime}=K$. (Observe that the two assumptions also hold for the quotient $G^{\prime} \stackrel{\text { def }}{=} G_{K^{\prime}} \rightarrow \mathfrak{G}^{\prime}=G^{\prime} / N$.) By Lemma 1.3, one has $\operatorname{cd}_{l}(N) \leq 1$. (See [Serre 1994, chapitre II, proposition 9], which only treats the number field case but whose proof works as it is in our function field case.) Next, by the assumption that $N^{l}=N$, one has $H^{1}(N, M)=\operatorname{Hom}(N, M)=0$. Thus, we have $H^{k}(G, M)=H^{k}\left(\mathfrak{G}, H^{0}(N, M)\right)=H^{k}(\mathfrak{G}, M)$, as desired.

Proposition 1.5 (Galois characterization of decomposition subgroups).

(i) Let $\tilde{x} \neq \tilde{x}^{\prime}$ be two elements of $\Sigma_{\tilde{X}}$. Then $\mathfrak{D}_{\tilde{x}} \cap \mathfrak{D}_{\tilde{x}^{\prime}}$ is of order prime to l, and hence, in particular, is open neither in $\mathfrak{D}_{\tilde{x}}$ nor in $\mathfrak{D}_{\tilde{x}^{\prime}}$.

(ii) Let $\operatorname{Dec}_{l}(\mathfrak{G}) \subset \operatorname{Sub}(\mathfrak{G})$ be the set of closed subgroups $\mathfrak{D}$ of $\mathfrak{G}$ satisfying the following property: There exists an open subgroup $\mathfrak{D}_{0}$ of $\mathfrak{D}$ such that for any open subgroup $\mathfrak{D}^{\prime} \subset \mathfrak{D}_{0}, \operatorname{dim}_{\mathbb{F}_{l}} H^{2}\left(\mathfrak{D}^{\prime}, \mathbb{F}_{l}\right)=1$. Define $\operatorname{Dec}_{l}^{\max }(\mathfrak{G}) \subset \operatorname{Dec}_{l}(\mathfrak{G})$ to be the set of maximal elements of $\operatorname{Dec}_{l}(\mathfrak{G})$. Then the map $\Sigma_{\tilde{X}} \rightarrow \operatorname{Sub}(\mathfrak{G}), \tilde{x} \mapsto \mathfrak{D}_{\tilde{x}}$ induces a bijection $\Sigma_{\tilde{X}} \stackrel{\sim}{\rightarrow} \operatorname{Dec}_{l}^{\max }(\mathfrak{G})$, and, in particular, is injective.

Proof. (i) As in [Uchida 1977], this follows from the approximation theorem [Neukirch 1969b, Lemma 8]. More precisely, let $\mathfrak{D}_{l}$ be an $l$-Sylow subgroup of $\mathfrak{D}_{\tilde{x}} \cap \mathfrak{D}_{\tilde{x}}$, and suppose that $\mathfrak{D}_{l} \neq 1$. Since $\mathfrak{D}_{l} \subset \mathfrak{D}_{\tilde{x}, l}$ is torsion-free, $\mathfrak{D}_{l}$ is an infinite group. Thus, one may replace $\mathfrak{G}$ by any open subgroup, and assume that $\zeta_{l} \in K$, that the images $x$ and $x^{\prime}$ in $\Sigma_{X}$ of $\tilde{x}$ and $\tilde{x}^{\prime}$ are distinct, and that the image of $\mathfrak{D}_{l}$ in $\mathfrak{G}^{\mathrm{ab}} /\left(\mathfrak{G}^{\mathrm{ab}}\right)^{l}$ is nontrivial. In particular, this implies that the natural map

$$
\mathfrak{D}_{\tilde{x}}^{\mathrm{ab}} /\left(\mathfrak{D}_{\tilde{x}}^{\mathrm{ab}}\right)^{l} \times \mathfrak{D}_{\tilde{x}^{\prime}}^{\mathrm{ab}} /\left(\mathfrak{D}_{\tilde{x}^{\prime}}^{\mathrm{ab}}\right)^{l} \rightarrow \mathfrak{G}^{\mathrm{ab}} /\left(\mathfrak{G}^{\mathrm{ab}}\right)^{l}
$$


is not injective. By Kummer theory, this last condition is equivalent to saying that the natural map

$$
K^{\times} /\left(K^{\times}\right)^{l} \rightarrow K_{x}^{\times} /\left(K_{x}^{\times}\right)^{l} \times K_{x^{\prime}}^{\times} /\left(K_{x^{\prime}}^{\times}\right)^{l}
$$

is not surjective. This contradicts the approximation theorem. (Note that $\left(K_{x}^{\times}\right)^{l}$ and $\left(K_{x^{\prime}}^{\times}\right)^{l}$ are open in $K_{x}^{\times}$and $K_{x^{\prime}}^{\times}$, respectively.)

(ii) By Proposition 1.1(i) and Lemmas 1.3 and 1.4, the proof of Uchida [1977] (which is essentially due to Neukirch, [1969a; 1969b]) works as it is. See [Uchida 1977, Lemmas 1-3] for more details.

Remark 1.6. For other characterizations of decomposition groups - applicable to much more general situations - see, for example, Theorem 1.16 of [Pop 1994], Theorem 2 of [Koenigsmann 2003], or the results in [Engler and Koenigsmann 1998; Engler and Nogueira 1994].

1D. Fundamental groups of curves. Write

$$
I \stackrel{\text { def }}{=}\left\langle I_{\tilde{\tilde{x}}}\right\rangle \tilde{\tilde{x} \in \Sigma_{\tilde{\tilde{X}}}}
$$

for the closed subgroup of $G$ generated by the inertia subgroups $I_{\tilde{\tilde{x}}}$ for all $\tilde{\tilde{x}} \in$ $\Sigma_{\tilde{X}}$, and call it the inertia subgroup of $G$. Then $I$ is normal in $G$. The quotient $G / I$ is canonically identified with the fundamental group $\pi_{1}(X)$ of $X$ with base point $\operatorname{Spec}(\bar{K}) \rightarrow X$ [Grothendieck and Raynaud 1971]. We have a natural exact sequence

$$
1 \rightarrow \pi_{1}(\bar{X}) \rightarrow \pi_{1}(X) \stackrel{\mathrm{pr}}{\rightarrow} G_{k} \rightarrow 1,
$$

where $\pi_{1}(\bar{X})$ is the fundamental group of $\bar{X} \stackrel{\text { def }}{=} X \times{ }_{k} \bar{k}$ with base point $\operatorname{Spec}(\bar{K}) \rightarrow \bar{X}$ and $\mathrm{pr}$ is the canonical projection. We have the exact sequence

$$
1 \rightarrow \pi_{1}(X)^{\mathrm{ab}, \text { tor }} \rightarrow \pi_{1}(X)^{\mathrm{ab}} \stackrel{\mathrm{pr}}{\rightarrow} G_{k} \rightarrow 1,
$$

where $\pi_{1}(X)^{\mathrm{ab} \text {,tor }}$ is the torsion subgroup of $\pi_{1}(X)^{\mathrm{ab}}$, and pr is the canonical projection. Moreover, $\pi_{1}(X)^{\mathrm{ab} \text {,tor }}$ is a finite abelian group that is canonically isomorphic to the group $J_{X}(k)$ of $k$-rational points of the Jacobian variety $J_{X}$ of $X$.

More generally, write $\mathfrak{I} \stackrel{\text { def }}{=}\left\langle\mathfrak{I}_{\tilde{x}}\right\rangle_{\tilde{x} \in \Sigma_{\tilde{X}}}$ for the closed subgroup of $\mathfrak{G}$ generated by the inertia subgroups $\mathfrak{I}_{\tilde{x}}$ for all $\tilde{x} \in \Sigma_{\tilde{X}}$, and call it the inertia subgroup of $\mathfrak{G}$. Then $\mathfrak{I}$ is normal in $\mathfrak{G}$. Set $\Pi_{X} \stackrel{\text { def }}{=} \mathfrak{G} / \mathfrak{I}$, which is a quotient of $\pi_{1}(X)$. Define $\Pi_{\bar{X}}$ to be the image of $\pi_{1}(\bar{X})$ in $\Pi_{X}$. Then we have a natural exact sequence

$$
1 \rightarrow \Pi_{\bar{X}} \rightarrow \Pi_{X} \stackrel{\mathrm{pr}}{\rightarrow} \mathfrak{G}_{k} \rightarrow 1 .
$$

When $\mathfrak{G}=G^{(\mathscr{C})}$ for a full class $\mathscr{C}$ of finite groups, we have $\Pi_{X}=\pi_{1}(X)^{(\mathscr{C})}$. In this case, we have the exact sequence:

$$
1 \rightarrow \Pi_{X}^{\mathrm{ab}, \mathrm{tor}} \rightarrow \Pi_{X}^{\mathrm{ab}} \stackrel{\mathrm{pr}}{\rightarrow} G_{k} \rightarrow 1
$$


where $\Pi_{X}^{\mathrm{ab}, \text { tor }}$ is the torsion subgroup of $\Pi_{X}^{\mathrm{ab}}$. Moreover, $\Pi_{X}^{\mathrm{ab}, \text { tor }}$ is a finite abelian group that is canonically isomorphic to the maximal (pro-) $\mathscr{C}$-quotient $J_{X}(k)^{\mathscr{C}}$ of the finite group $J_{X}(k)$.

\section{Basic properties of homomorphisms between Galois groups}

In this section we investigate some basic properties of homomorphisms between Galois groups of function fields of curves over finite fields. First, we shall investigate a class of homomorphisms between decomposition subgroups, which arise naturally from the class of homomorphisms between (quotients of) Galois groups that we consider in Sections 3 and 4.

\section{A. Homomorphisms between Galois groups of local fields of positive charac-} teristics. For $i \in\{1,2\}$, let $p_{i}>0$ be a prime number. Let $L_{i}$ be a complete discrete valuation field of equal characteristic $p_{i}$, with finite residue field $\ell_{i}$. Let $O_{L_{i}}$ be the ring of integers of $L_{i}$. Also, fix a separable closure $L_{i}^{\text {sep }}$ of $L_{i}$. We shall denote the residue field of $L_{i}^{\text {sep }}$ by $\bar{\ell}_{i}$, since it is an algebraic closure of $\ell_{i}$. Note that $\ell_{i}$ and $\bar{\ell}_{i}$ can also be regarded naturally as subfields of $L_{i}$ and $L_{i}^{\text {sep }}$, respectively. Write $D_{i} \stackrel{\text { def }}{=} \operatorname{Gal}\left(L_{i}^{\text {sep }} / L_{i}\right)$ for the corresponding absolute Galois group of $L_{i}$, and call $I_{i} \subset D_{i}$ the inertia subgroup. For each prime number $l$, let $D_{i, l}$ be an $l$-Sylow subgroup of $D_{i}$.

By local class field theory [Serre 1967], we have a natural isomorphism

$$
\left(L_{i}^{\times}\right)^{\wedge} \stackrel{\sim}{\rightarrow} D_{i}^{\mathrm{ab}},
$$

where $\left(L_{i}^{\times}\right)^{\wedge} \stackrel{\text { def }}{=}{\underset{n}{n}}_{\lim _{i}} L_{i}^{\times} /\left(L_{i}^{\times}\right)^{n}$. In particular, $D_{i}^{\text {ab }}$ fits into an exact sequence

$$
0 \rightarrow \mathrm{O}_{L_{i}}^{\times} \rightarrow D_{i}^{\mathrm{ab}} \rightarrow \hat{\mathbb{Z}} \rightarrow 0
$$

(arising from a similar exact sequence for $\left(L_{i}^{\times}\right)^{\wedge}$ ), where $0_{L_{i}}^{\times}$is the group of multiplicative units in $\mathscr{O}_{L_{i}}$. Moreover, we obtain natural inclusions

$$
\ell_{i}^{\times} \times U_{i}^{1}=\mathcal{O}_{L_{i}}^{\times} \subset L_{i}^{\times} \hookrightarrow D_{i}^{\mathrm{ab}},
$$

where $U_{i}^{1}$ is the group of principal units in $\mathscr{O}_{L_{i}}^{\times}$, and

$$
L_{i}^{\times} / \mathcal{O}_{L_{i}}^{\times} \stackrel{\sim}{\rightarrow} \mathbb{Z} \hookrightarrow D_{i}^{\mathrm{ab}} / \operatorname{Im}\left(\mathbb{O}_{L_{i}}^{\times}\right)
$$

(where $\stackrel{\sim}{\rightarrow}$ is the isomorphism induced by the valuation), by considering the Frobenius element.

Let $\mathfrak{D}_{i}$ be a quotient of $D_{i}, \mathfrak{I}_{i}$ the image of $I_{i}$ in $\mathfrak{D}_{i}$, and $\mathfrak{G}_{\ell_{i}} \stackrel{\text { def }}{=} \mathfrak{D}_{i} / \mathfrak{I}_{i}$. For each prime number $l$, let $\mathfrak{D}_{i, l}$ be the image of $D_{i, l}$ in $\mathfrak{D}_{i}$, which is an $l$-Sylow subgroup of $\mathfrak{D}_{i}$. Write

$$
\mathfrak{I m}\left(\ell_{i}^{\times}\right), \mathfrak{I m}\left(U_{i}^{1}\right) \subset \mathfrak{I m}\left(\mathbb{O}_{L_{i}}^{\times}\right) \subset \mathfrak{I m}\left(L_{i}^{\times}\right) \subset \mathfrak{D}_{i}^{\mathrm{ab}}
$$


for the images of $\ell_{i}^{\times}, U_{i}^{1}, \mathscr{O}_{L_{i}}^{\times}$and $L_{i}^{\times}$in $\mathfrak{D}_{i}^{\mathrm{ab}}$, respectively. In the rest of this subsection, we assume that either $\mathfrak{D}_{i}=D_{i}, i=1,2$ or $\mathfrak{D}_{i}=D_{i}^{\mathrm{t}}=D_{i}^{\left(p_{i}^{\prime}\right)}, i=$ 1,2 , and refer to the former and the latter cases as the profinite and the tame cases, respectively. Thus, we have $\mathfrak{D}_{i}^{\mathrm{ab}}=\left(L_{i}^{\times}\right)^{\wedge}, \mathfrak{I m}\left(L_{i}^{\times}\right)=L_{i}^{\times}, \mathfrak{I m}\left(\mathcal{O}_{L_{i}}^{\times}\right)=\mathcal{O}_{L_{i}}^{\times}$, $\mathfrak{I m}\left(\ell_{i}^{\times}\right)=\ell_{i}^{\times}$and $\mathfrak{I m}\left(U_{i}^{1}\right)=U_{i}^{1}$ in the profinite case, and $\mathfrak{D}_{i}^{\mathrm{ab}}=\left(L_{i}^{\times}\right)^{\wedge} / U_{i}^{1}$, $\mathfrak{I m}\left(L_{i}^{\times}\right)=L_{i}^{\times} / U_{i}^{1}, \mathfrak{I m}\left(\mathcal{O}_{L_{i}}^{\times}\right)=\mathcal{O}_{L_{i}}^{\times} / U_{i}^{1}=\mathfrak{I m}\left(\ell_{i}^{\times}\right)=\ell_{i}^{\times}$and $\mathfrak{I m}\left(U_{i}^{1}\right)=\{1\}$ in the tame case.

Let

$$
\tau: \mathfrak{D}_{1} \rightarrow \mathfrak{D}_{2}
$$

be a surjective homomorphism between profinite groups. Write $\tau^{\mathrm{ab}}: \mathfrak{D}_{1}^{\mathrm{ab}} \rightarrow \mathfrak{D}_{2}^{\mathrm{ab}}$ for the induced surjective homomorphism between the maximal abelian quotients. For each prime number $l, \tau\left(\mathfrak{D}_{1, l}\right)$ is an $l$-Sylow subgroup of $\mathfrak{D}_{2}$, and we shall assume that $\tau\left(\mathfrak{D}_{1, l}\right)=\mathfrak{D}_{2, l}$.

Proposition 2.1 (invariants of arbitrary surjective homomorphisms between decomposition groups). (i) The equality $p_{1}=p_{2}$ holds. Set $p \stackrel{\text { def }}{=} p_{1}=p_{2}$.

(ii) Let $l \neq p$ be a prime number. We have $\mathfrak{D}_{1, l} \cap \operatorname{Ker} \tau=\{1\}$. In particular, $\operatorname{Ker} \tau$ is pro- $p$. In the tame case, $\tau$ is an isomorphism.

(iii) The homomorphism $\tau$ induces a natural bijection $\ell_{1}^{\times} \stackrel{\sim}{\rightarrow} \ell_{2}^{\times}$between the multiplicative groups of residue fields. In particular, $\ell_{1}$ and $\ell_{2}$ have the same cardinality.

(iv) $\tau$ induces naturally an isomorphism $M_{\bar{\ell}_{1}} \stackrel{\sim}{\rightarrow} M_{\bar{\ell}_{2}}$, which is Galois-equivariant with respect to $\tau$. In particular, $\tau$ commutes with the cyclotomic characters $\chi_{i}: \mathfrak{D}_{i} \rightarrow\left(\hat{\mathbb{Z}}^{p^{\prime}}\right)^{\times}$of $\mathfrak{D}_{i}$, that is, the following diagram is commutative:

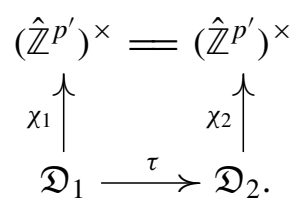

(v) We have $\tau\left(\mathfrak{I}_{1}\right)=\mathfrak{I}_{2}$.

(vi) The homomorphism $\tau^{\mathrm{ab}}: \mathfrak{D}_{1}^{\mathrm{ab}} \rightarrow \mathfrak{D}_{2}^{\mathrm{ab}}$ preserves $\mathfrak{I m}\left(L_{i}^{\times}\right), \mathfrak{I m}\left(\mathbb{O}_{L_{i}}^{\times}\right), \mathfrak{I m}\left(\ell_{i}^{\times}\right)$ and $\mathfrak{I m}\left(U_{i}^{1}\right)$. Further, the isomorphism $\mathfrak{D}_{1}^{\mathrm{ab}} / \mathfrak{I m}\left(\mathcal{O}_{L_{1}}^{\times}\right) \rightarrow \mathfrak{D}_{2}^{\mathrm{ab}} / \mathfrak{I m}\left(\mathbb{O}_{L_{2}}^{\times}\right)$induced by $\tau$ preserves the respective Frobenius elements.

Proof. Property (i) follows by considering the $q$-Sylow subgroups of $\mathfrak{D}_{i}$ for various prime numbers $q$. Indeed, for $i \in\{1,2\}, \mathfrak{D}_{i, p_{i}}$ is not (topologically) finitely generated (resp. is cyclic) in the profinite (resp. tame) case, while $\mathfrak{D}_{i, l}$ for a prime number $l \neq p_{i}$ is (topologically) finitely generated and noncyclic. Accordingly, the surjection $\mathfrak{D}_{1, p_{2}} \rightarrow \mathfrak{D}_{2, p_{2}}$ (resp. $\mathfrak{D}_{1, p_{1}} \rightarrow \mathfrak{D}_{2, p_{1}}$ ) cannot exist in the profinite (resp. tame) case, unless $p_{1}=p_{2}$. Thus, we must have $p_{1}=p_{2}$. 
The first assertion of (ii) follows from Proposition 1.1(i), applied to the quotient $D_{1} \rightarrow \mathfrak{D}_{1} \stackrel{\tau}{\rightarrow} \mathfrak{D}_{2}$. The second assertion follows from the first. The third assertion follows from the second, together with the fact (which can be checked easily) that $D_{1}^{\mathrm{t}}$ admits no nontrivial normal pro- $p$ subgroup.

Next, we prove (iii). By local class field theory, the torsion subgroup $\mathfrak{D}_{i}^{\mathrm{ab}, \text { tor }}$ of $\mathfrak{D}_{i}^{\mathrm{ab}}$ is naturally identified with $\ell_{i}{ }^{\times}$(both in the profinite and the tame cases), and hence, in particular, is finite of order prime to $p$. By (ii), the kernel of the surjective homomorphism $\tau^{\mathrm{ab}}: \mathfrak{D}_{1}^{\mathrm{ab}} \rightarrow \mathfrak{D}_{2}^{\mathrm{ab}}$ is pro- $p$. Thus, $\tau^{\mathrm{ab}}$ induces a natural isomorphism $\mathfrak{D}_{1}^{\text {ab,tor }} \stackrel{\sim}{\rightarrow} \mathfrak{D}_{2}^{\text {ab,tor }}$, which is naturally identified with $\ell_{1}^{\times} \stackrel{\sim}{\rightarrow} \ell_{2}^{\times}$, as desired.

By applying the above argument to open subgroups of $\mathfrak{D}_{i}$ (which correspond to each other via $\tau$ ), with $i=1,2$, and passing to the projective limit with respect to the norm maps, we obtain a natural isomorphism $M_{\bar{\ell}_{1}} \stackrel{\sim}{\rightarrow} M_{\bar{\ell}_{2}}$ between the modules of roots of unity. Here, we use the fact that if $L_{i}^{\prime}$ is a finite extension of $L_{i}$ corresponding to an open subgroup $\mathfrak{D}_{i}^{\prime}$ of $\mathfrak{D}_{i}$, then the following diagram commutes:

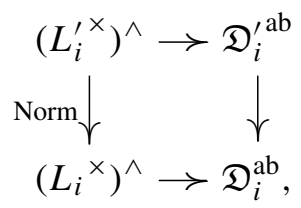

where the horizontal maps are the natural surjective homomorphisms from local class field theory, and the map $\mathfrak{D}_{i}^{\prime \text { ab }} \rightarrow \mathfrak{D}_{i}^{\mathrm{ab}}$ is induced by the natural inclusion $\mathfrak{D}_{i}^{\prime} \subset \mathfrak{D}_{i}$. Further, this identification is (by construction) Galois-compatible with respect to the homomorphism $\tau$. This completes the proof of (iv).

Property (v) follows from property (iv), since $\mathfrak{I}_{i}$ coincides with the kernel of $\chi_{i}$ for $i=1,2$.

Next, we prove (vi). First, $\tau^{\mathrm{ab}}$ preserves the image $\mathfrak{I m}\left(\mathcal{O}_{L_{i}}^{\times}\right)$by $(\mathrm{v})$, since this image coincides with the image of the inertia subgroup $\mathfrak{I}_{i}$. Since $\mathfrak{I m}\left(\ell_{i}^{\times}\right)$(resp. $\mathfrak{I m}\left(U_{i}^{1}\right)$ ) is the maximal prime-to- $p$ (resp. pro- $p$ ) subgroup of $\mathfrak{I m}\left(\mathcal{O}_{L_{i}}^{\times}\right)$, property (vi) for $\mathfrak{I m}\left(\ell_{i}^{\times}\right)$(resp. $\left.\mathfrak{I m}\left(U_{i}^{1}\right)\right)$ follows. Further, by (iii) and (iv), the homomorphism $\mathfrak{D}_{1}^{\mathrm{ab}} / \mathfrak{I m}\left(\mathbb{O}_{L_{1}}^{\times}\right) \rightarrow \mathfrak{D}_{2}^{\mathrm{ab}} / \mathfrak{I m}\left(\mathbb{O}_{L_{2}}^{\times}\right)$induced by $\tau$ preserves the respective Frobenius elements, since such an element is characterized as the unique element whose image under $\chi_{i}$ is $\sharp\left(\ell_{i}\right)$. Finally, since $\mathfrak{I m}\left(L_{i}^{\times}\right)$is the inverse image in $\mathfrak{D}_{i}^{\mathrm{ab}}$ of the subgroup generated by the Frobenius element in $\mathfrak{D}_{i}^{\mathrm{ab}} / \mathfrak{I m}\left(\mathcal{O}_{L_{i}}^{\times}\right)$for $i=1,2$, they are preserved by $\tau^{\mathrm{ab}}$.

\section{B. Homomorphisms between Galois groups of function fields of curves over} finite fields. Next, we shall investigate some basic properties of homomorphisms between Galois groups of function fields of curves over finite fields. We follow the notations in Section 1, especially subsections 1A and 1B. Moreover: 
Notation. (i) For $i \in\{1,2\}$, let $k_{i}$ be a finite field of characteristic $p_{i}>0$. Let $X_{i}$ be a smooth, proper, geometrically connected curve of genus $g_{i} \geq 0$ over $k_{i}$. Let $K_{i}=K_{X_{i}}$ be the function field of $X_{i}$ and fix an algebraic closure $\bar{K}_{i}$ of $K_{i}$. Let $K_{i}^{\text {sep }}$ be the separable closure of $K_{i}$ in $\bar{K}_{i}$, and $\bar{k}_{i}$ the algebraic closure of $k_{i}$ in $\bar{K}_{i}$. Following the notations in Section 1 , we will write $G_{i} \stackrel{\text { def }}{=} G_{K_{i}}=\operatorname{Gal}\left(K_{i}^{\text {sep }} / K_{i}\right)$ for the absolute Galois group of $K_{i}$, and $\bar{G}_{i} \stackrel{\text { def }}{=} G_{K_{i} \bar{k}_{i}}=\operatorname{Gal}\left(K_{i}^{\text {sep }} / K_{i} \bar{k}_{i}\right)$ for the absolute Galois group of $K_{i} \bar{k}_{i}$.

(ii) Let $N_{i}$ be a normal closed subgroup of $G_{i}$ and set $\mathfrak{G}_{i} \stackrel{\text { def }}{=} G_{i} / N_{i}$. Let $\tilde{K}_{i}$ denote the Galois extension of $K_{i}$ corresponding to $N_{i}$, that is, $\tilde{K}_{i} \stackrel{\text { def }}{=}\left(K_{i}^{\text {sep }}\right)^{N_{i}}$. Let $\bar{G}_{i}$ be the image of $\bar{G}_{i}$ in $\mathfrak{G}_{\mathfrak{i}}$, and set $\mathfrak{G}_{k_{i}} \stackrel{\text { def }}{=} \mathfrak{G}_{i} / \overline{\mathfrak{G}}_{i}$, which is a quotient of $G_{k_{i}}=\operatorname{Gal}\left(\bar{k}_{i} / k_{i}\right)$. For $i=1,2$, let us denote by $\varphi_{k_{i}}$ the image in $\mathfrak{G}_{k_{i}}$ of the $\sharp\left(k_{i}\right)$-th power Frobenius element of $G_{k_{i}}$.

(iii) Write $\tilde{X}_{i}$ for the integral closure of $X_{i}$ in $\tilde{K}_{i}$. The Galois group $\mathfrak{G}_{i}$ acts naturally on the set $\Sigma_{\tilde{X}_{i}}$, and the quotient $\Sigma_{\tilde{X}_{i}} / \mathfrak{G}_{i}$ is naturally identified with $\Sigma_{X_{i}}$. Denote the natural quotient map $\Sigma_{\tilde{X}_{i}} \rightarrow \Sigma_{X_{i}}$ by $q_{i}$. For a point $\tilde{x}_{i} \in \Sigma_{\tilde{X}_{i}}$, with residue field $k_{i}\left(\tilde{x}_{i}\right)$ (which is naturally identified with a subfield of $\bar{k}_{i}$ ), we define its decomposition group $\mathfrak{D}_{\tilde{x}_{i}}$ and inertia group $\mathfrak{I}_{\tilde{x}_{i}}$ by

$$
\mathfrak{D}_{\tilde{x}_{i}} \stackrel{\text { def }}{=}\left\{\gamma \in \mathfrak{G}_{i} \mid \gamma\left(\tilde{x}_{i}\right)=\tilde{x}_{i}\right\}
$$

and

$$
\mathfrak{I}_{\tilde{x}_{i}} \stackrel{\text { def }}{=}\left\{\gamma \in \mathfrak{D}_{\tilde{x}_{i}} \mid \gamma \text { acts trivially on } k_{i}\left(\tilde{x}_{i}\right)\right\},
$$

respectively. Set $\mathfrak{G}_{k_{i}\left(x_{i}\right)} \stackrel{\text { def }}{=} \mathfrak{D}_{\tilde{x}_{i}} / \mathfrak{I}_{\tilde{x}_{i}}$, where $x_{i}$ stands for the image of $\tilde{x}_{i}$ in $\Sigma_{X_{i}}$.

Write $\mathfrak{I}_{i} \stackrel{\text { def }}{=}\left\langle I_{\tilde{x}_{i}}\right\rangle_{\tilde{x}_{i} \in \Sigma_{\tilde{X}_{i}}}$ for the closed subgroup of $\mathfrak{G}_{i}$ generated by the inertia subgroups $\mathfrak{I}_{\tilde{x}_{i}}$ for all $\tilde{x}_{i} \in \Sigma_{\tilde{X}_{i}}$, and call it the inertia subgroup of $\mathfrak{G}_{i}$. Then $\mathfrak{I}_{i}$ is normal in $\mathfrak{G}_{i}$.

(iv) Let $\sigma: \mathfrak{G}_{1} \rightarrow \mathfrak{G}_{2}$ be a continuous homomorphism between profinite groups.

Proposition 2.2 (image of a decomposition subgroup). Let $l \neq p_{1}, p_{2}$ be a prime number, and assume that (1) $N_{2}^{l}=N_{2}$, or, equivalently, $\tilde{K}_{2}$ admits no l-cyclic extension; and (2) $\tilde{K}_{2}$ contains a primitive l-th root of unity. For each $\tilde{x}_{1} \in \Sigma_{\tilde{X}_{1}}$, fix an l-Sylow subgroup $\mathfrak{D}_{\tilde{x}_{1}, l}$ of $\mathfrak{D}_{\tilde{x}_{1}}$ and set $\mathfrak{I}_{\tilde{x}_{1}, l} \stackrel{\text { def }}{=} \mathfrak{I}_{\tilde{x}_{1}} \cap \mathfrak{D}_{\tilde{x}_{1}, l}$, which is an l-Sylow subgroup of $\mathfrak{I}_{\tilde{x}_{1}}$. Let $\Sigma_{\tilde{X}_{1}, \sigma, l}$ be the set of $\tilde{x}_{1} \in \Sigma_{\tilde{X}_{1}}$ such that $\operatorname{cd} l\left(\sigma\left(\mathfrak{D}_{\tilde{x}_{1}}\right)\right)=2$. Then:

(i) There exists a unique map $\tilde{\phi}=\tilde{\phi}_{\sigma, l}: \Sigma_{\tilde{X}_{1}, \sigma, l} \rightarrow \Sigma_{\tilde{X}_{2}}$ such that $\sigma\left(\mathfrak{D}_{\tilde{x}_{1}}\right) \subset \mathfrak{D}_{\tilde{\phi}\left(\tilde{x}_{1}\right)}$ for each $\tilde{x}_{1} \in \Sigma_{\tilde{X}_{1}, \sigma, l}$.

(ii) For each $\tilde{x}_{1} \in \Sigma_{\tilde{X}_{1}, \sigma, l}$, there exists an l-Sylow subgroup $\mathfrak{D}_{\tilde{\phi}\left(\tilde{x}_{1}\right), l}$ of $\mathfrak{D}_{\tilde{\phi}\left(\tilde{x}_{1}\right)}$ such that $\sigma\left(\mathfrak{D}_{\tilde{x}_{1}, l}\right) \subset \mathfrak{D}_{\tilde{\phi}\left(\tilde{x}_{1}\right), l}$. Moreover, we have $\sigma\left(\mathfrak{I}_{\tilde{x}_{1}, l}\right) \subset \mathfrak{I}_{\tilde{\phi}\left(\tilde{x}_{1}\right), l}$, where we set

$$
\mathfrak{I}_{\tilde{\phi}\left(\tilde{x}_{1}\right), l} \stackrel{\text { def }}{=} \mathfrak{I}_{\tilde{\phi}\left(\tilde{x}_{1}\right)} \cap \mathfrak{D}_{\tilde{\phi}\left(\tilde{x}_{1}\right), l},
$$


which is an l-Sylow subgroup of $\mathfrak{I}_{\tilde{\phi}\left(\tilde{x}_{1}\right)}$.

(iii) The subset $\Sigma_{\tilde{X}_{1}, \sigma, l} \subset \Sigma_{\tilde{X}_{1}}$ is $\mathfrak{G}_{1}$-stable, or, equivalently,

$$
\Sigma_{\tilde{X}_{1}, \sigma, l}=q_{1}^{-1}\left(\Sigma_{X_{1}, \sigma, l}\right),
$$

where $\Sigma_{X_{1}, \sigma, l} \stackrel{\text { def }}{=} q_{1}\left(\Sigma_{\tilde{X}_{1}, \sigma, l}\right)$. The map $\tilde{\phi}$ is Galois-compatible with respect to $\sigma$ : we have

$$
\tilde{\phi}\left(g_{1} \tilde{x}_{1}\right)=\sigma\left(g_{1}\right) \tilde{\phi}\left(\tilde{x}_{1}\right)
$$

for any $\tilde{x}_{1} \in \Sigma_{\tilde{X}_{1}, \sigma, l}$ and any $g_{1} \in \mathfrak{G}_{1}$. In particular, $\tilde{\phi}$ induces naturally a $\operatorname{map} \phi=\phi_{\sigma, l}: \Sigma_{X_{1}, \sigma, l} \rightarrow \Sigma_{X_{2}}$.

(iv) For any $\tilde{x}_{1} \in \Sigma_{\tilde{X}_{1}} \backslash \Sigma_{\tilde{X}_{1}, \sigma, l}$, we have $\sigma\left(\mathfrak{I}_{\tilde{x}_{1}, l}\right)=\{1\}$.

(v) For two primes $l=l_{1}, l_{2}$ satisfying the assumptions, $\tilde{\phi}_{\sigma, l_{1}}$ and $\tilde{\phi}_{\sigma, l_{2}}$ coincide with each other on the intersection $\Sigma_{\tilde{X}_{1}, \sigma, l_{1}} \cap \Sigma_{\tilde{X}_{1}, \sigma, l_{2}}$.

Proof.

(i) Take $\tilde{x}_{1} \in \Sigma_{\tilde{X}_{1}, \sigma, l}$. Applying Proposition 1.1(i)(ii) to $\mathfrak{D}=\sigma\left(\mathfrak{D}_{\tilde{x}_{1}}\right)$, we have $\sigma\left(\mathfrak{D}_{\tilde{x}_{1}}\right) \in \operatorname{Dec}_{l}\left(\mathfrak{G}_{2}\right)$ in the notation of the result in part (ii) of Proposition 1.5. Thus, by this same result, there exists $\tilde{x}_{2} \in \Sigma_{\tilde{X}_{2}}$ such that $\sigma\left(\mathfrak{D}_{\tilde{x}_{1}}\right) \subset \mathfrak{D}_{\tilde{x}_{2}}$. By Proposition 1.5(i), such $\tilde{x}_{2}$ is unique. So, set

$$
\tilde{\phi}\left(\tilde{x}_{1}\right)=\tilde{x}_{2},
$$

which has the desired properties.

(ii) The existence of $\mathfrak{D}_{\tilde{\phi}\left(\tilde{x}_{1}\right), l}$ follows from the fact that $\sigma\left(\mathfrak{D}_{\tilde{x}_{1}, l}\right) \subset \sigma\left(\mathfrak{D}_{\tilde{x}_{1}}\right) \subset \mathfrak{D}_{\tilde{\phi}\left(\tilde{x}_{1}\right)}$ and that $\sigma\left(\mathfrak{D}_{\tilde{x}_{1}, l}\right)$ is pro-l. Finally, consider the composite map of

$$
\mathfrak{D}_{\tilde{x}_{1}} \stackrel{\sigma}{\rightarrow} \mathfrak{D}_{\tilde{\phi}\left(\tilde{x}_{1}\right)} \rightarrow \mathfrak{D}_{\tilde{\phi}\left(\tilde{x}_{1}\right)} / \mathfrak{I}_{\tilde{\phi}\left(\tilde{x}_{1}\right)}=\mathfrak{G}_{k_{2}\left(q_{2}\left(\tilde{\phi}\left(\tilde{x}_{1}\right)\right)\right)} .
$$

Then, since $\operatorname{cd}_{l}\left(\mathfrak{G}_{k_{2}\left(q_{2}\left(\tilde{\phi}\left(\tilde{x}_{1}\right)\right)\right)}\right)=1$, the image of $\mathfrak{I}_{\tilde{x}_{1}, l}$ in $\mathfrak{G}_{k_{2}\left(q_{2}\left(\tilde{\phi}\left(\tilde{x}_{1}\right)\right)\right)}$ must be trivial by Proposition 1.1(i), as desired.

(iii) Immediate from the definitions.

(iv) We have $\operatorname{cd}_{l}\left(\sigma\left(\mathfrak{D}_{\tilde{x}_{1}}\right)\right) \leq \operatorname{cd}_{l}\left(\mathfrak{G}_{2}\right) \leq 2<\infty$, where the second inequality follows from Lemma 1.4. Now, the assertion follows from Proposition 1.1(i).

(v) This follows from the fact that the defining property $\sigma\left(\mathfrak{D}_{\tilde{x}_{1}}\right) \subset \mathfrak{D}_{\tilde{\phi}\left(\tilde{x}_{1}\right)}$ of $\tilde{\phi}$ is independent of $l$.

We shall consider the following conditions:

Condition 1. Either $\mathfrak{G}_{i}=G_{i}, i=1,2$ or $\mathfrak{G}_{i}=G_{i}^{\left(p_{i}^{\prime}\right)}, i=1,2$. We refer to the former and the latter cases as the profinite and the prime-to-characteristic cases, respectively. (Observe that conditions (1) and (2) in Proposition 2.2 are then satisfied for any prime number $l \neq p_{1}, p_{2}$.) In particular, we have $\mathfrak{G}_{k_{i}}=G_{k_{i}}$ in both cases. 
Condition 2. The map $\sigma: \mathfrak{G}_{1} \rightarrow \mathfrak{G}_{2}$ commutes with the projections $\mathrm{pr}_{1}, \mathrm{pr}_{2}$, that is, it inserts into the commutative diagram

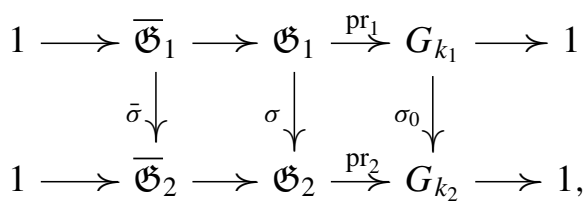

where the rows are exact.

Condition 3. The map $\sigma: \mathfrak{G}_{1} \rightarrow \mathfrak{G}_{2}$ is an open homomorphism.

In the rest of this section, we assume that Condition 1 holds.

Lemma 2.3. In the prime-to-characteristic case, Condition 2 automatically holds. In the profinite case, if $\sigma\left(\mathfrak{I}_{1}\right) \subseteq \mathfrak{I}_{2}$, then Condition 2 holds.

Proof. In the prime-to-characteristic case, the quotient $\mathrm{pr}_{i}: \mathfrak{G}_{i} \rightarrow G_{k_{i}}$ coincides with $\mathfrak{G}_{i}^{\mathrm{ab}}$ modulo the closure of the torsion subgroup. Thus, $\sigma$ commutes with the projections $\mathrm{pr}_{1}, \mathrm{pr}_{2}$.

In the profinite case, assume that $\sigma\left(\mathfrak{I}_{1}\right) \subseteq \mathfrak{I}_{2}$. Then $\sigma$ induces naturally, by passing to the quotients $\mathfrak{G}_{i} / \mathfrak{I}_{i}$, a homomorphism $\pi_{1}\left(X_{1}\right) \rightarrow \pi_{1}\left(X_{2}\right)$ between fundamental groups. The quotient $\mathrm{pr}_{i}: \mathfrak{G}_{i} \rightarrow \pi_{1}\left(X_{i}\right) \rightarrow G_{k_{i}}$ coincides with $\pi_{1}\left(X_{i}\right)^{\mathrm{ab}}$ modulo the torsion subgroup. Thus, $\sigma$ commutes with the projections $\mathrm{pr}_{1}, \mathrm{pr}_{2}$.

In the rest of this section, we assume, moreover, that Condition 3 holds. Then note that, if Condition 2 also holds and if $\sigma_{0}: G_{k_{1}} \rightarrow G_{k_{2}}$ and $\bar{\sigma}: \overline{\mathfrak{G}}_{1} \rightarrow \overline{\mathfrak{G}}_{2}$ are homomorphisms induced by $\sigma$, then automatically $\sigma_{0}$ is open and injective and $\bar{\sigma}$ is open.

Lemma 2.4 (invariance of the characteristics). The equality $p_{1}=p_{2}$ holds.

Proof. By replacing $\mathfrak{G}_{2}$ by the open subgroup $\sigma\left(\mathfrak{G}_{1}\right) \subset \mathfrak{G}_{2}$, we may and shall assume that $\sigma$ is surjective.

In the profinite case, the assertion follows by considering the (pro-) $q$-parts of $\mathfrak{G}_{i}^{\mathrm{ab}}$ for various prime numbers $q$. More precisely, for $i \in\{1,2\}$, consider the filtration $\mathfrak{G}_{i}^{\mathrm{ab}}=F_{i}^{0} \supset F_{i}^{1} \supset F_{i}^{2}$, where $F_{i}^{1}$ is the image of $\overline{\mathfrak{G}}_{i}=\operatorname{Ker}\left(\mathfrak{G}_{i} \rightarrow G_{k_{i}}\right)$ and $F_{i}^{2}$ is the image of $\operatorname{Ker}\left(\mathfrak{G}_{i} \rightarrow \pi_{1}\left(X_{i}\right)\right)$. Then, by global class field theory, $F_{i}^{0} / F_{i}^{1}=G_{k_{i}}(\simeq \hat{\mathbb{Z}}), F_{i}^{1} / F_{i}^{2}=J_{X_{i}}\left(k_{i}\right)$ (finite), and

$$
F_{i}^{2}=\left(\prod_{x_{i} \in \Sigma_{X_{i}}} \hat{\mathrm{O}}_{X_{i}, x_{i}}^{\times}\right) / k_{i}^{\times},
$$

where $\hat{O}_{X_{i}, x_{i}}^{\times}$is the multiplicative group of the completed local ring of $X_{i}$ at $x_{i}$. Further, we have a natural decomposition $\hat{O}_{X_{i}, x_{i}}^{\times}=k\left(x_{i}\right)^{\times} \times U_{x_{i}}^{1}$, where $k\left(x_{i}\right)^{\times}$is the multiplicative group of the residue field of $X_{i}$ at $x_{i}$ (and hence finite) and $U_{x_{i}}^{1}$ is 
the group of principal units in $\hat{O}_{X_{i}, x_{i}}^{\times}$(and hence isomorphic to a direct product of countably infinite copies of $\mathbb{Z}_{p_{i}}$ ). Therefore, the $p_{i}$-part of $\mathfrak{G}_{i}^{\mathrm{ab}}$ modulo the closure of the torsion subgroup is not finitely generated, while the $l$-part of $\mathfrak{G}_{i}^{\text {ab }}$ modulo the closure of the torsion subgroup, for a prime number $l \neq p_{i}$, is finitely generated (and even cyclic). (Note, however, that the $l$-torsion subgroup of $\mathfrak{G}_{i}^{\text {ab }}$ is infinite.) Thus, $\mathfrak{G}_{2}$ being a quotient of $\mathfrak{G}_{1}($ via $\sigma)$ we must have $p_{1}=p_{2}$.

In the prime-to-characteristic case, the assertion follows by considering the $q$ Sylow subgroups $\mathfrak{G}_{i, q}$ of $\mathfrak{G}_{i}$ for various prime numbers $q$. As $\sigma$ is assumed to be surjective, we may and shall take $\mathfrak{G}_{2, q}=\sigma\left(\mathfrak{G}_{1, q}\right)$. Indeed, for $i \in\{1,2\}, \mathfrak{G}_{i, p_{i}}$ is cyclic, while $\mathfrak{G}_{i, l}$ for a prime number $l \neq p_{i}$ is noncyclic. Accordingly, the surjection $\mathfrak{G}_{1, p_{1}} \rightarrow \mathfrak{G}_{2, p_{1}}$ cannot exist, unless $p_{1}=p_{2}$. Thus $p_{1}=p_{2}$.

So, from now on, set $p \stackrel{\text { def }}{=} p_{1}=p_{2}$.

Remark 2.5. The same argument used in the proof of (the prime-to-characteristic case of) Lemma 2.3 shows that an open homomorphism $\sigma: \mathfrak{G}_{1} \rightarrow \mathfrak{G}_{2}$ between profinite groups automatically commutes with the natural projections $\operatorname{pr}_{i}^{\prime}: \mathfrak{G}_{i} \rightarrow G_{k_{i}}^{p^{\prime}}$, induced by $\operatorname{pr}_{i}$, for $i=1,2$. Thus, we have the commutative diagram

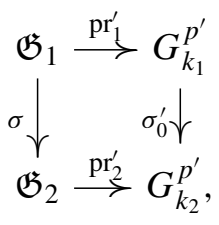

where the right column is automatically open and injective. The authors do not know, at least at the time of writing, whether or not Condition 2 follows from Conditions 1 and 3 in general (that is, even in the profinite case).

In the rest of this subsection we assume that Condition 2 holds.

Lemma 2.6. The map $\sigma$ induces a natural open homomorphism $\sigma^{\prime}: G_{1}^{\left(p^{\prime}\right)} \rightarrow G_{2}^{\left(p^{\prime}\right)}$, which commutes with the canonical projections

$$
G_{i}^{\left(p^{\prime}\right)} \rightarrow G_{k_{i}} ; \quad i=1,2 .
$$

For $i=1,2$, let $\mathfrak{I}_{i}^{\prime}$ be the image of $\mathfrak{I}_{i} \subset \mathfrak{G}_{i}$ in $G_{i}^{\left(p^{\prime}\right)}$. Then $\sigma^{\prime}\left(\mathfrak{I}_{1}^{\prime}\right) \subset \mathfrak{I}_{2}^{\prime}$. Thus, $\sigma$ induces a natural open homomorphism $\tau^{\prime}: \pi_{1}\left(X_{1}\right)^{\left(p^{\prime}\right)} \rightarrow \pi_{1}\left(X_{2}\right)^{\left(p^{\prime}\right)}$, which commutes with the canonical projections $\pi_{1}\left(X_{i}\right)^{\left(p^{\prime}\right)} \rightarrow G_{k_{i}} ; i=1$, 2. In particular, we have $g_{1} \geq g_{2}$.

Proof. The first assertion is clear. The second follows from Proposition 2.2(ii)(iv). The third assertion follows from the second. Now, $\tau^{\prime}: \pi_{1}\left(X_{1}\right)^{\left(p^{\prime}\right)} \rightarrow \pi_{1}\left(X_{2}\right)^{\left(p^{\prime}\right)}$ induces an open homomorphism $\pi_{1}\left(\bar{X}_{1}\right)^{p^{\prime}} \rightarrow \pi_{1}\left(\bar{X}_{2}\right)^{p^{\prime}}$, and hence an open homo-

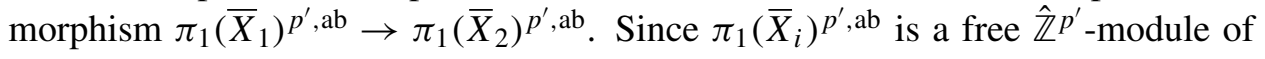
rank $2 g_{i}$ for $i=1,2$, this implies the last assertion. 
Lemma 2.7. For a prime number $l \neq p$, the map $\phi=\phi_{\sigma, l}: \Sigma_{X_{1}, \sigma, l} \rightarrow \Sigma_{X_{2}}$ is almost surjective, that is, $\Sigma_{X_{2}} \backslash \phi\left(\Sigma_{X_{1}, \sigma, l}\right)$ is finite. In particular, $\Sigma_{X_{1}, \sigma, l}$ is infinite (and hence, a fortiori, nonempty).

Proof. Assume that the set $S \stackrel{\text { def }}{=} \Sigma_{X_{2}} \backslash \phi\left(\Sigma_{X_{1}, \sigma, l}\right)$ is infinite. Set $U_{2} \stackrel{\text { def }}{=} X_{2} \backslash S$.

As in (the third assertion of) Lemma 2.6, then $\sigma$ induces an open homomorphism $\tau_{1}^{(l)}: \pi_{1}\left(X_{1}\right)^{(l)} \rightarrow \pi_{1}\left(U_{2}\right)^{(l)}$, which is a lifting of the homomorphism $\tau^{(l)}$ : $\pi_{1}\left(X_{1}\right)^{(l)} \rightarrow \pi_{1}\left(X_{2}\right)^{(l)}$ induced by $\tau^{\prime}: \pi_{1}\left(X_{1}\right)^{\left(p^{\prime}\right)} \rightarrow \pi_{1}\left(X_{2}\right)^{\left(p^{\prime}\right)}$. We have a commutative diagram

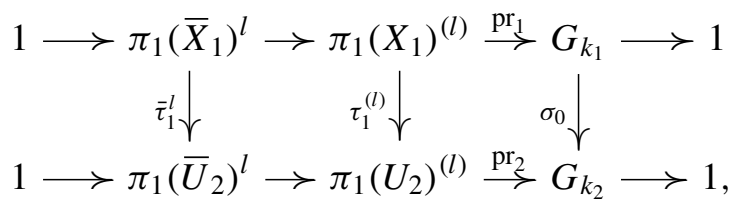

where $\bar{U}_{2} \stackrel{\text { def }}{=} U_{2} \times_{k_{2}} \bar{k}_{2}$. Since $\tau_{1}^{(l)}: \pi_{1}\left(X_{1}\right)^{(l)} \rightarrow \pi_{1}\left(U_{2}\right)^{(l)}$ is open and $\sigma_{0}: G_{k_{1}} \rightarrow G_{k_{2}}$ is (open and) injective, we see that $\bar{\tau}_{1}^{l}: \pi_{1}\left(\bar{X}_{1}\right)^{l} \rightarrow \pi_{1}\left(\bar{U}_{2}\right)^{l}$ is open. This is a contradiction, since $\pi_{1}\left(\bar{X}_{1}\right)^{l}$ is (topologically) finitely generated, while $\pi_{1}\left(\bar{U}_{2}\right)^{l}$ (and hence $\bar{\tau}_{1}^{l}\left(\pi_{1}\left(\bar{X}_{1}\right)^{l}\right)$ also) is not (topologically) finitely generated, since $S$ is infinite.

Lemma 2.8. Let $\sigma_{0}: G_{k_{1}} \rightarrow G_{k_{2}}$ be the (open, injective) homomorphism induced by $\sigma$. Set $d_{0} \stackrel{\text { def }}{=}\left[G_{k_{2}}: \sigma_{0}\left(G_{k_{1}}\right)\right]$.

(i) The following diagram is commutative:

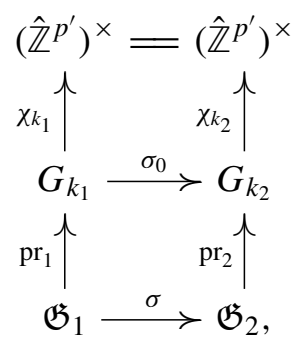

where $\chi_{k_{i}}$ is the cyclotomic character of $G_{k_{i}}$ for $i=1,2$.

(ii) We have $\sharp\left(k_{1}\right)=\sharp\left(k_{2}\right)^{d_{0}}$ and $\sigma_{0}\left(\varphi_{k_{1}}\right)=\varphi_{k_{2}}^{d_{0}}$.

Proof. (i) Since the bottom square is commutative by the definition of $\sigma_{0}$, we only have to prove that the top square is commutative. As $G_{k_{2}}$ is (topologically) generated by $\varphi_{k_{2}}$, we may write $\sigma_{0}\left(\varphi_{k_{1}}\right)=\varphi_{k_{2}}^{\alpha}$, where $\alpha \in \hat{\mathbb{Z}}$. Now, the desired commutativity $\chi_{k_{2}} \circ \sigma_{0}=\chi_{k_{1}}$ is equivalent to saying that $\chi_{k_{2}}\left(\sigma_{0}\left(\varphi_{k_{1}}\right)\right)=\chi_{k_{1}}\left(\varphi_{k_{1}}\right)$ (as $G_{k_{1}}$ is (topologically) generated by $\left.\varphi_{k_{1}}\right)$. Since $\chi_{k_{1}}\left(\varphi_{k_{1}}\right)=\sharp\left(k_{1}\right)=p^{\left[k_{1}: \mathbb{F}_{p}\right]}$ and

$$
\chi_{k_{2}}\left(\sigma_{0}\left(\varphi_{k_{1}}\right)\right)=\chi_{k_{2}}\left(\varphi_{k_{2}}^{\alpha}\right)=\chi_{k_{2}}\left(\varphi_{k_{2}}\right)^{\alpha}=\sharp\left(k_{2}\right)^{\alpha}=p^{\alpha\left[k_{2}: \mathbb{F}_{p}\right]},
$$


the desired commutativity is thus equivalent to the equality $\alpha\left[k_{2}: \mathbb{F}_{p}\right]=\left[k_{1}: \mathbb{F}_{p}\right]$ in $\hat{\mathbb{Z}}$. (The homomorphism $\hat{\mathbb{Z}} \rightarrow\left(\hat{\mathbb{Z}}^{p^{\prime}}\right)^{\times}, \beta \mapsto p^{\beta}$ is injective by [Chevalley 1951, théorème 1].) In particular, it suffices to prove the desired commutativity on an open subgroup $H \subset G_{k_{1}}$. Indeed, set $m \stackrel{\text { def }}{=}\left[G_{k_{1}}: H\right]$. Then, since $\varphi_{k_{1}}^{m}$ is the Frobenius element for $H$, the commutativity on $H$ is equivalent to the equality $m \alpha\left[k_{2}: \mathbb{F}_{p}\right]=m\left[k_{1}: \mathbb{F}_{p}\right]$ in $\hat{\mathbb{Z}}$, which implies $\alpha\left[k_{2}: \mathbb{F}_{p}\right]=\left[k_{1}: \mathbb{F}_{p}\right]$, as desired. Thus, by replacing $\mathfrak{G}_{1}$ and $\mathfrak{G}_{2}$ by suitable open subgroups, we may and shall assume that $g_{2}>0$.

Next, for each prime number $l \neq p$ and $i \in\{1,2\}$, let $\chi_{k_{i}, l}: G_{k_{i}} \rightarrow \mathbb{Z}_{l}^{\times}$denote the $l$-adic cyclotomic character. Thus, corresponding to the decomposition $\left(\hat{\mathbb{Z}}^{p^{\prime}}\right)^{\times}=$ $\prod_{l \neq p} \mathbb{Z}_{l}^{\times}$, we have $\chi_{k_{i}}=\left(\chi_{k_{i}, l}\right)_{l \neq p}$. We have to prove that $\chi_{k_{2}} \circ \sigma_{0}=\chi_{k_{1}}$, which is equivalent to saying that $\chi_{k_{2}, l} \circ \sigma_{0}=\chi_{k_{1}, l}$ for all $l \neq p$.

We shall first prove that the last equality holds up to torsion. More precisely, denote by $\bar{\chi}_{k_{i}, l}$ the composite of

$$
G_{k_{i}} \stackrel{\chi_{k_{i}, l}}{\rightarrow} \mathbb{Z}_{l}^{\times} \rightarrow \mathbb{Z}_{l}^{\times} /\left(\mathbb{Z}_{l}^{\times}\right)^{\text {tor }}
$$

By Lemma 2.7, we can take $\tilde{x}_{1} \in \Sigma_{\tilde{X}_{1}, \sigma, l} \neq \varnothing$. Set $\tilde{x}_{2} \stackrel{\text { def }}{=} \tilde{\phi}\left(\tilde{x}_{1}\right)$. Let $x_{i}$ denote the image of $\tilde{x}_{i}$ in $\Sigma_{X_{i}}$ for $i=1,2$. By Proposition 2.2(ii), we have $\sigma: \mathfrak{D}_{\tilde{x}_{1}, l} \rightarrow \mathfrak{D}_{\tilde{x}_{2}, l}$ and $\sigma: \mathfrak{I}_{\tilde{x}_{1}, l} \rightarrow \mathfrak{I}_{\tilde{x}_{2}, l}$, which are injective by Proposition 1.1(i). This implies that $\chi_{k_{2}, l} \circ \sigma_{0}=\chi_{k_{1}, l}$ holds on the image of $\mathfrak{D}_{\tilde{x}_{1}, l}$ in $G_{k_{1}}$, which is an open subgroup of the $l$-Sylow subgroup $G_{k_{1}, l}$ of $G_{k_{1}}$. As $\mathbb{Z}_{l}^{\times} /\left(\mathbb{Z}_{l}^{\times}\right)^{\text {tor }} \simeq \mathbb{Z}_{l}$ is torsion-free and pro-l, this implies that $\bar{\chi}_{k_{2}, l} \circ \sigma_{0}=\bar{\chi}_{k_{1}, l}$.

In particular, we have $\bar{\chi}_{k_{2}, l}\left(\sigma_{0}\left(\varphi_{k_{1}}\right)\right)=\bar{\chi}_{k_{1}, l}\left(\varphi_{k_{1}}\right)$. This implies the equality $\sharp\left(k_{2}\right)^{\alpha}=\sharp\left(k_{1}\right)$ in $\mathbb{Z}_{l}^{\times} /\left(\mathbb{Z}_{l}^{\times}\right)^{\text {tor }} \simeq \mathbb{Z}_{l}$. Since $p \in \mathbb{Z}_{l}^{\times}$is not torsion, this last equality shows that $\alpha_{l}\left[k_{2}: \mathbb{F}_{p}\right]=\left[k_{1}: \mathbb{F}_{p}\right]$ in $\mathbb{Z}_{l}$. Here, corresponding to the decomposition

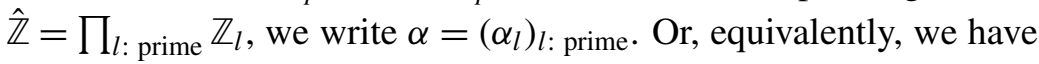

$$
\alpha\left[k_{2}: \mathbb{F}_{p}\right]=\left[k_{1}: \mathbb{F}_{p}\right]+\iota_{p}(\epsilon)
$$

in $\hat{\mathbb{Z}}$, where $\iota_{p}: \mathbb{Z}_{p} \hookrightarrow \hat{\mathbb{Z}}$ is the natural injection and $\epsilon \stackrel{\text { def }}{=} \alpha_{p}\left[k_{2}: \mathbb{F}_{p}\right]-\left[k_{1}: \mathbb{F}_{p}\right] \in \mathbb{Z}_{p}$.

On the other hand, by Lemma 2.6, we get an open homomorphism $\pi_{1}\left(\bar{X}_{1}\right)^{p^{\prime}} \rightarrow$ $\pi_{1}\left(\bar{X}_{2}\right)^{p^{\prime}}$, and hence a surjection $\pi_{1}\left(\bar{X}_{1}\right)^{p^{\prime}, \mathrm{ab}} \otimes_{\mathbb{Z}} \mathbb{Q} \rightarrow \pi_{1}\left(\bar{X}_{2}\right)^{p^{\prime}, \mathrm{ab}} \otimes_{\mathbb{Z}} \mathbb{Q}$, which is Galois-compatible with respect to

$$
\sigma_{0}: G_{k_{1}} \rightarrow G_{k_{2}}
$$

For each $i=1,2$, let $P_{i}(T)$ be the characteristic polynomial of $\varphi_{k_{i}}^{\left[k_{l^{\prime}}: \mathbb{F}_{p}\right]}$ on the free $\hat{\mathbb{Z}}^{p^{\prime}}$-module $\pi_{1}\left(\bar{X}_{i}\right)^{p^{\prime}}$,ab (of rank $2 g_{i}$ ), where $i^{\prime}$ is defined by $\left\{i, i^{\prime}\right\}=\{1,2\}$. Then it is known that $P_{i}(T) \in \mathbb{Z}[T]$.

Write $\rho_{i}$ for the natural representation $G_{k_{i}} \rightarrow \operatorname{Aut}_{\widehat{\mathbb{Z}}^{p^{\prime}}}\left(\pi_{1}\left(\bar{X}_{i}\right)^{p^{\prime}, \text { ab }}\right)$. Let $R_{\mathbb{Q}}$ be

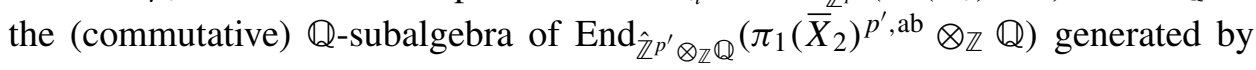


$\rho_{2}\left(G_{k_{2}}\right)$. We have

$$
P_{2}\left(\rho_{2}\left(\varphi_{k_{2}}^{\left[k_{1}: \mathbb{F}_{p}\right]}\right)\right)=0
$$

in $R_{\mathbb{Q}}$. By the Galois-compatibility, we also have

$$
P_{1}\left(\rho_{2}\left(\sigma_{0}\left(\varphi_{k_{1}}^{\left[k_{2}: \mathbb{F}_{p}\right]}\right)\right)\right)=0
$$

in $R_{\mathbb{Q}}$. These identities imply that both of $\rho_{2}\left(\varphi_{k_{2}}^{\left[k_{1}: \mathbb{F}_{p}\right]}\right), \rho_{2}\left(\sigma_{0}\left(\varphi_{k_{2}}^{\left[k_{2}: \mathbb{F}_{p}\right]}\right)\right) \in R_{\mathbb{Q}}$ are algebraic over $\mathbb{Q}$, and hence so is the ratio

$$
\rho_{2}\left(\sigma_{0}\left(\varphi_{k_{1}}^{\left[k_{2}: \mathbb{E}_{p}\right]}\right)\left(\varphi_{k_{2}}^{\left[k_{1}: \mathbb{F}_{p}\right]}\right)^{-1}\right)=\rho_{2}\left(\varphi_{k_{2}}^{\alpha\left[k_{2}: \mathbb{F}_{p}\right]-\left[k_{1}: \mathbb{E}_{p}\right]}\right)=\rho_{2}\left(\varphi_{k_{2}}^{l_{p}(\epsilon)}\right) \stackrel{\text { def }}{=} \eta
$$

in $R_{\mathbb{Q}}$. So, take a monic polynomial $Q(T) \in \mathbb{Q}[T]$ satisfying $Q(\eta)=0$ in $R_{\mathbb{Q}}$. Set $b \stackrel{\text { def }}{=} \operatorname{deg}(Q)$.

Let $l \neq p$ be a prime number, and let $R_{l, \mathbb{Q}}$ be the image of $R_{\mathbb{Q}}$ in

$$
\operatorname{End}_{\mathbb{Q}_{l}}\left(\pi_{1}\left(\bar{X}_{2}\right)^{l, \mathrm{ab}} \otimes_{\mathbb{Z}} \mathbb{Q}\right) .
$$

Then observe that the image $\eta_{l}$ of $\eta$ in $R_{l, \mathbb{Q}} \subset \operatorname{End}_{\mathbb{Q}_{l}}\left(\pi_{1}\left(\bar{X}_{2}\right)^{l, \text { ab }} \otimes_{\mathbb{Z}} \mathbb{Q}\right)$ is a pro- $p$ element of $\operatorname{End}_{\mathbb{Z}_{l}}\left(\pi_{1}\left(\bar{X}_{2}\right)^{l, \mathrm{ab}}\right)^{\times}$, and hence a torsion element of $p$-power order. So, let $p^{a_{l}}$ be the order of $\eta_{l}$. As $Q\left(\eta_{l}\right)=0$ in the commutative $\mathbb{Q}$-algebra $R_{l, \mathbb{Q}}$, we conclude: $((p-1) / p) p^{a_{l}} \leq \varphi\left(p^{a_{l}}\right) \leq b$, where $\varphi$ stands for Euler's function. (Use $\mathbb{Q} \hookrightarrow R_{l, \mathbb{Q}}$, which follows from $g_{2}>0$.) Thus, $a_{l}$ is bounded: there exists $a \geq 0$ such that $a_{l} \leq a$ for all $l \neq p$. Namely, $\left(\eta_{l}\right)^{p^{a}}=1$ for all $l \neq p$.

Set $\zeta_{l} \stackrel{\text { def }}{=} \operatorname{det}\left(\eta_{l}\right)$, where the determinant is taken as an element of

$$
\operatorname{End}_{\mathbb{Q}_{l}}\left(\pi_{1}\left(\bar{X}_{2}\right)^{l, \mathrm{ab}} \otimes_{\mathbb{Z}} \mathbb{Q}\right) .
$$

Since det is a multiplicative homomorphism, we have $\left(\zeta_{l}\right)^{p^{a}}=1$ for all $l \neq p$. Set $\zeta \stackrel{\text { def }}{=}\left(\zeta_{l}\right)_{l \neq p}$ in $\left(\hat{\mathbb{Z}}^{p^{\prime}}\right)^{\times}=\prod_{l \neq p} \mathbb{Z}_{l}^{\times}$. Now, by construction, we have

$$
\zeta=\chi_{k_{2}}^{g_{2}}\left(\varphi_{k_{2}}^{\iota_{p}(\epsilon)}\right)=\sharp\left(k_{2}\right)^{g_{2} \iota_{p}(\epsilon)},
$$

and hence $\sharp\left(k_{2}\right)^{p^{a} g_{2} \iota_{p}(\epsilon)}=1$ in $\left(\hat{\mathbb{Z}}^{p^{\prime}}\right)^{\times}$. Since the homomorphism $\hat{\mathbb{Z}} \rightarrow\left(\hat{\mathbb{Z}}^{p^{\prime}}\right)^{\times}$, $\beta \mapsto p^{\beta}$ is injective, this last equality forces $\left[k_{2}: \mathbb{F}_{p}\right] p^{a} g_{2} \iota_{p}(\epsilon)=0$ in $\hat{\mathbb{Z}}$. As $\left[k_{2}: \mathbb{F}_{p}\right] p^{a} g_{2}>0$, this implies $\iota_{p}(\epsilon)=0$. Namely, we have $\alpha\left[k_{2}: \mathbb{F}_{p}\right]=\left[k_{1}: \mathbb{F}_{p}\right]$ in $\hat{\mathbb{Z}}$, as desired.

(ii) As in the proof of (i), set $\sigma_{0}\left(\varphi_{k_{1}}\right)=\varphi_{k_{2}}^{\alpha}$. Since $G_{k_{2}} \simeq \hat{\mathbb{Z}}$ and $\left[G_{k_{2}}: \sigma_{0}\left(G_{k_{1}}\right)\right]=$ $d_{0}$, we must have $\alpha=d_{0} u$, where $u \in \hat{\mathbb{Z}}^{\times}$. Now, since $\alpha\left[k_{2}: \mathbb{F}_{p}\right]=\left[k_{1}: \mathbb{F}_{p}\right]$ by (i), we get $d_{0} u\left[k_{2}: \mathbb{F}_{p}\right]=\left[k_{1}: \mathbb{F}_{p}\right]$, and thus $u=\left[k_{1}: \mathbb{F}_{p}\right] /\left(d_{0}\left[k_{2}: \mathbb{F}_{p}\right]\right) \in \mathbb{Q}_{>0}\left(\subset \mathbb{Z} \otimes_{\mathbb{Z}} \mathbb{Q}\right)$. Since $\hat{\mathbb{Z}}^{\times} \cap \mathbb{Q}_{>0}=\{1\}$, we conclude $u=1$. Thus, $d_{0}\left[k_{2}: \mathbb{F}_{p}\right]=\left[k_{1}: \mathbb{F}_{p}\right]$ and $\sigma_{0}\left(\varphi_{k_{1}}\right)=\varphi_{k_{2}}^{d_{0}}$, as desired.

Lemma 2.9. For each prime number $l \neq p$, the map $\tilde{\phi}_{\sigma, l}: \Sigma_{\tilde{X}_{1}, \sigma, l} \rightarrow \Sigma_{\tilde{X}_{2}}$ is surjective. In particular, the map $\phi_{\sigma, l}: \Sigma_{X_{1}, \sigma, l} \rightarrow \Sigma_{X_{2}}$ is surjective. 
Proof. As in the proof of Lemma 2.7, set

$$
S \stackrel{\text { def }}{=} \Sigma_{X_{2}} \backslash \phi\left(\Sigma_{X_{1}, \sigma, l}\right) \text { and } \quad U_{2} \stackrel{\text { def }}{=} X_{2} \backslash S .
$$

By Lemma 2.7, $S$ is a finite set. Let $r<\infty$ be the cardinality of $S\left(\bar{k}_{2}\right)$. Then $\sigma$ induces an open homomorphism $\tau_{1}^{(l)}: \pi_{1}\left(X_{1}\right)^{(l)} \rightarrow \pi_{1}\left(U_{2}\right)^{(l)}$, which is a lifting of the homomorphism $\tau^{(l)}: \pi_{1}\left(X_{1}\right)^{(l)} \rightarrow \pi_{1}\left(X_{2}\right)^{(l)}$ induced by $\tau^{\prime}: \pi_{1}\left(X_{1}\right)^{\left(p^{\prime}\right)} \rightarrow$ $\pi_{1}\left(X_{2}\right)^{\left(p^{\prime}\right)}$ in Lemma 2.6. We have a commutative diagram

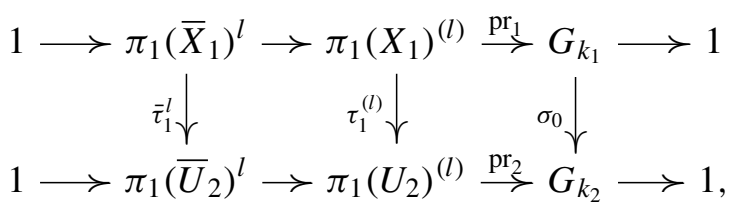

where $\bar{U}_{2} \stackrel{\text { def }}{=} U_{2} \times_{k_{2}} \bar{k}_{2}$. Since $\tau_{1}^{(l)}: \pi_{1}\left(X_{1}\right)^{(l)} \rightarrow \pi_{1}\left(U_{2}\right)^{(l)}$ is open and $\sigma_{0}: G_{k_{1}} \rightarrow G_{k_{2}}$ is (open and) injective, we see that $\bar{\tau}_{1}^{l}: \pi_{1}\left(\bar{X}_{1}\right)^{l} \rightarrow \pi_{1}\left(\bar{U}_{2}\right)^{l}$ is open. The open homomorphism $\bar{\tau}_{1}^{l}: \pi_{1}\left(\bar{X}_{1}\right)^{l} \rightarrow \pi_{1}\left(\bar{U}_{2}\right)^{l}$ induces an open homomorphism $\bar{\tau}_{1}^{l, \text { ab }}$ : $\pi_{1}\left(\bar{X}_{1}\right)^{l, \mathrm{ab}} \rightarrow \pi_{1}\left(\bar{U}_{2}\right)^{l, \mathrm{ab}}$. This last homomorphism is, by construction, Galoiscompatible with respect to $\sigma_{0}: G_{k_{1}} \rightarrow G_{k_{2}}$. In other words, if we regard $\pi_{1}\left(\bar{U}_{2}\right)^{l, \text { ab }}$ as a $G_{k_{1}}$-module via $\sigma_{0}$, then $\bar{\tau}_{1}^{l \text {,ab }}$ is a homomorphism as $G_{k_{1}}$-modules.

The absolute values of eigenvalues of $\varphi_{k_{1}} \in G_{k_{1}}$ in $\pi_{1}\left(\bar{X}_{1}\right)^{l, \text { ab }}$ are all $\sharp\left(k_{1}\right)^{1 / 2}$, with multiplicity $2 g_{1}$. On the other hand, by Lemma 2.8(ii), the absolute values of eigenvalues of $\varphi_{k_{1}}$ in $\pi_{1}\left(\bar{U}_{2}\right)^{l, \text { ab }}$ are the same as those of $\varphi_{k_{2}}^{d_{0}}$, which are $\sharp\left(k_{2}\right)^{d_{0} / 2}$ with multiplicity $2 g_{2}$ and $\sharp\left(k_{2}\right)^{d_{0}}$ with multiplicity $\max (r-1,0)$. By Lemma 2.8(i), they coincide with $\sharp\left(k_{1}\right)^{1 / 2}$ and $\sharp\left(k_{1}\right)$, respectively. Thus, we conclude $r \leq 1$. However, if $r \neq 0$, by replacing $\mathfrak{G}_{1}, \mathfrak{G}_{2}$ with suitable open subgroups, we may assume that $r>1$, a contradiction. So, we have established $r=0$.

To prove the surjectivity of $\tilde{\phi}_{\sigma, l}$, we may freely replace $\mathfrak{G}_{1}, \mathfrak{G}_{2}$ by open subgroups $\mathfrak{H}_{1}, \mathfrak{H}_{2}$, respectively, such that $\sigma\left(\mathfrak{H}_{1}\right) \subset \mathfrak{H}_{2}$. (Indeed, the map

$$
\tilde{\phi}_{\sigma, l}: \Sigma_{\tilde{X}_{1}, \sigma, l} \rightarrow \Sigma_{\tilde{X}_{2}}
$$

remains unchanged.) In particular, we may assume that $\sigma: \mathfrak{G}_{1} \rightarrow \mathfrak{G}_{2}$ is surjective. Then the surjectivity of $\tilde{\phi}_{\sigma, l}: \Sigma_{\tilde{X}_{1}, \sigma, l} \rightarrow \Sigma_{\tilde{X}_{2}}$ is equivalent to the surjectivity of $\phi_{\sigma, l}: \Sigma_{X_{1}, \sigma, l} \rightarrow \Sigma_{X_{2}}$, which is then equivalent to $r=0$.

\section{Rigid homomorphisms between Galois groups}

In this section we investigate a class of homomorphisms between (geometrically prime-to-characteristic quotients of) absolute Galois groups of function fields of curves over finite fields, which we call rigid. We follow the notations in Sections 1 and 2. In particular, we follow the Notation at the beginning of subsection 2B. We assume that Condition 3 holds. 
Definition 3.1 (rigid homomorphisms). (i) We say that $\sigma: \mathfrak{G}_{1} \rightarrow \mathfrak{G}_{2}$ is strictly rigid if there exists a map

$$
\tilde{\phi}: \Sigma_{\tilde{X}_{1}} \rightarrow \Sigma_{\tilde{X}_{2}}
$$

such that

$$
\sigma\left(\mathfrak{D}_{\tilde{x}_{1}}\right)=\mathfrak{D}_{\tilde{\phi}\left(\tilde{x}_{1}\right)}
$$

for each $\tilde{x}_{1} \in \Sigma_{\tilde{X}_{1}}$.

(ii) We say that $\sigma: \mathfrak{G}_{1} \rightarrow \mathfrak{G}_{2}$ is rigid if there exist open subgroups $\mathfrak{H}_{1} \subset \mathfrak{G}_{1}$, $\mathfrak{H}_{2} \subset \mathfrak{G}_{2}$, such that $\sigma\left(\mathfrak{H}_{1}\right) \subset \mathfrak{H}_{2}$ and that $\mathfrak{H}_{1} \stackrel{\sigma}{\rightarrow} \mathfrak{H}_{2}$ is strictly rigid. (Here, $\mathfrak{H}_{i}$ is considered as a quotient of the absolute Galois group that is the inverse image in $G_{i}$ of $\mathfrak{H}_{i} \subset \mathfrak{G}_{i}$.)

(iii) Define $\operatorname{Hom}\left(\mathfrak{G}_{1}, \mathfrak{G}_{2}\right)^{\text {rig }} \subset \operatorname{Hom}\left(\mathfrak{G}_{1}, \mathfrak{G}_{2}\right)$ to be the set of rigid (and hence continuous and open) homomorphisms $\mathfrak{G}_{1} \rightarrow \mathfrak{G}_{2}$.

Remark 3.2. (i) Consider a commutative diagram of maps between profinite groups

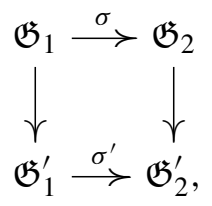

where the vertical arrows are surjective. Then if $\sigma: \mathfrak{G}_{1} \rightarrow \mathfrak{G}_{2}$ is strictly rigid (resp. rigid), $\sigma^{\prime}: \mathfrak{G}_{1}^{\prime} \rightarrow \mathfrak{G}_{2}^{\prime}$ is strictly rigid (resp. rigid).

(ii) Let $\mathfrak{H}_{2}$ be an open subgroup of $\mathfrak{G}_{2}$ and $\mathfrak{H}_{1} \stackrel{\text { def }}{=} \sigma^{-1}\left(\mathfrak{H}_{2}\right)$. Then if $\sigma: \mathfrak{G}_{1} \rightarrow \mathfrak{G}_{2}$ is strictly rigid (resp. rigid), the natural homomorphism $\mathfrak{H}_{1} \rightarrow \mathfrak{H}_{2}$ induced by $\sigma$ is strictly rigid (resp. rigid).

(iii) Assume that $\sigma: \mathfrak{G}_{1} \rightarrow \mathfrak{G}_{2}$ is strictly rigid with respect to $\tilde{\phi}: \Sigma_{\tilde{X}_{1}} \rightarrow \Sigma_{\tilde{X}_{2}}$. Then if $\tilde{\phi}$ is surjective, $\sigma$ is surjective. Indeed, this follows immediately from the fact, by Chebotarev's density theorem, that $\mathfrak{G}_{2}$ is (topologically) generated by its decomposition subgroups.

(iv) As in Proposition 2.2, let $l \neq p_{1}, p_{2}$ be a prime number, and assume that (1) $N_{2}^{l}=N_{2}$, or, equivalently, $\tilde{K}_{2}$ admits no $l$-cyclic extension; and (2) $\tilde{K}_{2}$ contains a primitive $l$-th roots of unity.

If $\sigma$ is strictly rigid with respect to $\tilde{\phi}: \Sigma_{\tilde{X}_{1}} \rightarrow \Sigma_{\tilde{X}_{2}}$, then we must have $\Sigma_{\tilde{X}_{1}, \sigma, l}=\Sigma_{\tilde{X}_{1}}$ and $\tilde{\phi}=\tilde{\phi}_{\sigma, l}$. In particular, then $\tilde{\phi}$ is unique and Galoisequivariant with respect to $\sigma$, and hence naturally induces a map $\phi\left(=\phi_{\sigma, l}\right)$ : $\Sigma_{X_{1}} \rightarrow \Sigma_{X_{2}}$.

If $\sigma$ is rigid, then we must have $\Sigma_{\tilde{X}_{1}, \sigma, l}=\Sigma_{\tilde{X}_{1}}$, and, if we set $\tilde{\phi} \stackrel{\text { def }}{=} \tilde{\phi}_{\sigma, l}$, then

$$
\sigma\left(\mathfrak{D}_{\tilde{x}_{1}}\right) \underset{\text { open }}{\subset} \mathfrak{D}_{\tilde{\phi}\left(\tilde{x}_{1}\right)}
$$


for each $\tilde{x}_{1} \in \Sigma_{\tilde{X}_{1}}$. The map $\tilde{\phi}$ is uniquely characterized by this property, and Galois-equivariant with respect to $\sigma$, and hence naturally induces a map

$$
\phi\left(=\phi_{\sigma, l}\right): \Sigma_{X_{1}} \rightarrow \Sigma_{X_{2}} .
$$

In the rest of this section, we assume that Condition 1 holds.

Definition 3.3. (i) Let $\gamma: K_{2} \rightarrow K_{1}$ be a homomorphism of fields defining an extension $K_{1} / K_{2}$ of fields. Set $p \stackrel{\text { def }}{=} p_{1}=p_{2}$. Then we say that $\gamma$ is admissible if the extension $K_{1} / K_{2}$ appears in the extensions of $K_{2}$ corresponding to the open subgroups of $\mathfrak{G}_{2}$. More precisely, in the profinite case, we say that $\gamma$ is admissible if the extension $K_{1} / K_{2}$ is finite separable; in the prime-to-characteristic case, we say that $\gamma$ is admissible if the extension $K_{1} / K_{2}$ is finite separable and the Galois closure of the extension $K_{1} \bar{k}_{1} / K_{2} \bar{k}_{2}$ is of degree prime to $p$.

Equivalently, $\gamma: K_{2} \rightarrow K_{1}$ is admissible if and only if it extends to an isomorphism $\tilde{\gamma}: \tilde{K}_{2} \stackrel{\sim}{\rightarrow} \tilde{K}_{1}$.

(ii) Define $\operatorname{Hom}\left(K_{2}, K_{1}\right)^{\mathrm{adm}} \subset \operatorname{Hom}\left(K_{2}, K_{1}\right)$ to be the set of admissible homomorphisms $K_{2} \rightarrow K_{1}$.

Our aim in this section is to prove the following.

Theorem 3.4. The natural map $\operatorname{Hom}\left(K_{2}, K_{1}\right) \rightarrow \operatorname{Hom}\left(\mathfrak{G}_{1}, \mathfrak{G}_{2}\right) / \operatorname{Inn}\left(\mathfrak{G}_{2}\right)$ induces a bijection

$$
\operatorname{Hom}\left(K_{2}, K_{1}\right)^{\mathrm{adm}} \stackrel{\sim}{\rightarrow} \operatorname{Hom}\left(\mathfrak{G}_{1}, \mathfrak{G}_{2}\right)^{\mathrm{rig}} / \operatorname{Inn}\left(\mathfrak{G}_{2}\right) .
$$

More precisely,

(i) If $\gamma: K_{2} \rightarrow K_{1}$ is an admissible homomorphism between fields, then the homomorphism $\mathfrak{G}_{1} \rightarrow \mathfrak{G}_{2}$ induced by $\gamma$ (up to inner automorphisms) is rigid.

(ii) If $\sigma: \mathfrak{G}_{1} \rightarrow \mathfrak{G}_{2}$ is a rigid homomorphism between profinite groups, then there exists a unique isomorphism $\tilde{\gamma}: \tilde{K}_{2} \rightarrow \tilde{K}_{1}$ of fields, such that $\tilde{\gamma} \circ \sigma\left(g_{1}\right)=g_{1} \circ \tilde{\gamma}$ for all $g_{1} \in \mathfrak{G}_{1}$, which induces an admissible homomorphism $K_{2} \rightarrow K_{1}$.

Remark 3.5. (i) By local theory for the Isom-form, any isomorphism $\mathfrak{G}_{1} \stackrel{\sim}{\rightarrow} \mathfrak{G}_{2}$ is strictly rigid. In particular, we have $\operatorname{Isom}\left(\mathfrak{G}_{1}, \mathfrak{G}_{2}\right) \subset \operatorname{Hom}\left(\mathfrak{G}_{1}, \mathfrak{G}_{2}\right)^{\text {rig }}$. Thus, Theorem 3.4 can be viewed as a generalization of the Isom-form:

$$
\operatorname{Isom}\left(K_{2}, K_{1}\right) \stackrel{\sim}{\rightarrow} \operatorname{Isom}\left(\mathfrak{G}_{1}, \mathfrak{G}_{2}\right) / \operatorname{Inn}\left(\mathfrak{G}_{2}\right),
$$

which is the main theorem of [Uchida 1977] in the profinite case, and the main theorem of [Saïdi and Tamagawa 2009] in the prime-to-characteristic case.

(ii) Let

$$
\gamma: K_{2}^{\text {perf }} \rightarrow K_{1}^{\text {perf }}
$$

be a homomorphism of fields defining an extension $K_{1}^{\text {perf }} / K_{2}^{\text {perf }}$ of fields. Set $p \stackrel{\text { def }}{=} p_{1}=p_{2}$. We say that $\gamma$ is admissible if the extension $K_{1}^{\text {perf }} / K_{2}^{\text {perf }}$ appears 
in the extensions of $K_{2}^{\text {perf }}$ corresponding to the open subgroups of $\mathfrak{G}_{2}$, which is regarded as a quotient of the absolute Galois group $G_{K_{2}^{\text {perf }}}=G_{K_{2}}$. More precisely, in the profinite case $\gamma$ is always admissible, and in the prime-to-characteristic case $\gamma$ is admissible if and only if the extension the Galois closure of the extension $K_{1}^{\text {perf }} \bar{k}_{1} / K_{2}^{\text {perf }} \bar{k}_{2}$ is of degree prime to $p$. Define

$$
\operatorname{Hom}\left(K_{2}^{\text {perf }}, K_{1}^{\text {perf }}\right)^{\text {adm }} \subset \operatorname{Hom}\left(K_{2}^{\text {perf }}, K_{1}^{\text {perf }}\right)
$$

to be the set of admissible homomorphisms $K_{2}^{\text {perf }} \rightarrow K_{1}^{\text {perf }}$. Then the natural map $\operatorname{Hom}\left(K_{2}^{\text {perf }}, K_{1}^{\text {perf }}\right) \rightarrow \operatorname{Hom}\left(\mathfrak{G}_{1}, \mathfrak{G}_{2}\right) / \operatorname{Inn}\left(\mathfrak{G}_{2}\right)$ induces a bijection

$$
\operatorname{Hom}\left(K_{2}^{\text {perf }}, K_{1}^{\text {perf }}\right)^{\text {adm }} / \operatorname{Frob}^{\mathbb{Z}} \stackrel{\sim}{\rightarrow} \operatorname{Hom}\left(\mathfrak{G}_{1}, \mathfrak{G}_{2}\right)^{\text {rig }} / \operatorname{Inn}\left(\mathfrak{G}_{2}\right) .
$$

Indeed, this follows from Theorem 3.4, since the natural map $\operatorname{Hom}\left(K_{2}, K_{1}\right) \rightarrow$ $\operatorname{Hom}\left(K_{2}^{\text {perf }}, K_{1}^{\text {perf }}\right)$ induces

$$
\operatorname{Hom}\left(K_{2}, K_{1}\right)^{\mathrm{adm}} \stackrel{\sim}{\rightarrow} \operatorname{Hom}\left(K_{2}^{\text {perf }}, K_{1}^{\text {perf }}\right)^{\text {adm }} / \text { Frob }^{\mathbb{Z}} .
$$

The rest of this section is devoted to the proof of Theorem 3.4.

First, to prove 3.4(i), let $\gamma: K_{2} \rightarrow K_{1}$ be an admissible homomorphism. Then, by the definition of admissibility, the extension $K_{1} / K_{2}$ is isomorphic to some extension $L / K_{2}$ that corresponds to an open subgroup $\mathfrak{H}_{2}$ of $\mathfrak{G}_{2}$. Set $\mathfrak{H}_{1} \stackrel{\text { def }}{=} \mathfrak{G}_{1}$. Now let $\sigma: \mathfrak{G}_{1} \rightarrow \mathfrak{G}_{2}$ be the homomorphism induced by $\gamma$ (up to conjugacy). Then it is easy to see that $\sigma$ restricts to an isomorphism $\mathfrak{H}_{1} \stackrel{\sim}{\rightarrow} \mathfrak{H}_{2}$ (corresponding to the isomorphism $L \stackrel{\sim}{\rightarrow} K_{1}$ ), which is strictly rigid. Thus, $\sigma$ is rigid, as desired.

Next, to prove 3.4(ii), let $\sigma: \mathfrak{G}_{1} \rightarrow \mathfrak{G}_{2}$ be a rigid homomorphism. By definition, there exist open subgroups $\mathfrak{H}_{1} \subset \mathfrak{G}_{1}, \mathfrak{H}_{2} \subset \mathfrak{G}_{2}$, such that $\sigma\left(\mathfrak{H}_{1}\right) \subset \mathfrak{H}_{2}$ and that $\mathfrak{H}_{1} \stackrel{\sigma}{\rightarrow} \mathfrak{H}_{2}$ is strictly rigid with respect to, say, $\tilde{\phi}: \Sigma_{\tilde{X}_{1}} \rightarrow \Sigma_{\tilde{X}_{2}}$. Then, by Remark 3.2(iv), $\tilde{\phi}$ is Galois-equivariant with respect to $\sigma: \mathfrak{G}_{1} \rightarrow \mathfrak{G}_{2}$ (that is, not only with respect to $\left.\sigma: \mathfrak{H}_{1} \rightarrow \mathfrak{H}_{2}\right)$, and, for each $\tilde{x}_{1} \in \Sigma_{\tilde{X}_{1}}$, we have $\sigma\left(\mathfrak{D}_{\tilde{x}_{1}}\right) \subset \mathfrak{D}_{\tilde{\phi}\left(\tilde{x}_{1}\right)}$ and $\sigma\left(\mathfrak{D}_{\tilde{x}_{1}} \cap \mathfrak{H}_{1}\right)=\mathfrak{D}_{\tilde{\phi}\left(\tilde{x}_{1}\right)} \cap \mathfrak{H}_{2}$.

Lemma 3.6. Condition 2 holds for $\sigma: \mathfrak{G}_{1} \rightarrow \mathfrak{G}_{2}$.

Proof. By Proposition 2.1(v), we have $\sigma\left(\mathfrak{I}_{\tilde{x}_{1}}\right) \subset \mathfrak{I}_{\tilde{\phi}\left(\tilde{x}_{1}\right)}$ for each $\tilde{x}_{1} \in \Sigma_{\tilde{X}_{1}}$. In particular, we have $\sigma\left(\mathfrak{I}_{1}\right) \subset \mathfrak{I}_{2}$. Now, the assertion follows from Lemma 2.3.

Thus, we may apply Lemmas 2.6-2.9 to $\sigma$.

Lemma 3.7. We have $\sigma\left(\mathfrak{H}_{1}\right)=\mathfrak{H}_{2}$ and $\mathfrak{H}_{1}=\sigma^{-1}\left(\mathfrak{H}_{2}\right)$.

Proof. By Lemma 2.9, $\tilde{\phi}$ is surjective, and hence, by Remark 3.2(iii), $\sigma: \mathfrak{H}_{1} \rightarrow \mathfrak{H}_{2}$ is surjective, that is, $\sigma\left(\mathfrak{H}_{1}\right)=\mathfrak{H}_{2}$.

Next, let $X_{1, \mathfrak{H}_{1}} \rightarrow X_{1, \sigma^{-1}\left(\mathfrak{H}_{2}\right)} \rightarrow X_{1}$ and $X_{2, \mathfrak{H}_{2}} \rightarrow X_{2}$ be (finite, generically étale) covers corresponding to open subgroups $\mathfrak{H}_{1} \subset \sigma^{-1}\left(\mathfrak{H}_{2}\right) \subset \mathfrak{G}_{1}$ and $\mathfrak{H}_{2} \subset \mathfrak{G}_{2}$, 
respectively. Suppose that $\mathfrak{H}_{1} \subsetneq \sigma^{-1}\left(\mathfrak{H}_{2}\right)$. Then, by Chebotarev's density theorem, there exists $\tilde{x}_{1} \in \Sigma_{\tilde{X}_{1}}$ such that

$$
k\left(x_{1, \mathfrak{H}_{1}}\right) \supsetneq k\left(x_{1, \sigma^{-1}\left(\mathfrak{H}_{2}\right)}\right),
$$

where $x_{1, \mathfrak{H}_{1}}$ and $x_{1, \sigma^{-1}\left(\mathfrak{H}_{2}\right)}$ denote the images of $\tilde{x}_{1}$ in $\Sigma_{1, \mathfrak{H}_{1}}$ and $\Sigma_{1, \sigma^{-1}\left(\mathfrak{H}_{2}\right)}$, respectively. Set $\tilde{x}_{2} \stackrel{\text { def }}{=} \tilde{\phi}\left(\tilde{x}_{1}\right) \in \Sigma_{\tilde{X}_{2}}$. We have $\sigma\left(\mathfrak{D}_{\tilde{x}_{1}}\right) \subset \mathfrak{D}_{\tilde{x}_{2}}$, and hence

$$
\sigma\left(\mathfrak{D}_{\tilde{x}_{1}} \cap \mathfrak{H}_{1}\right) \subset \sigma\left(\mathfrak{D}_{\tilde{x}_{1}} \cap \sigma^{-1}\left(\mathfrak{H}_{2}\right)\right) \subset \mathfrak{D}_{\tilde{x}_{2}} \cap \mathfrak{H}_{2} .
$$

Now, since $\mathfrak{H}_{1} \stackrel{\sigma}{\rightarrow} \mathfrak{H}_{2}$ is strictly rigid, we must have

$$
\sigma\left(\mathfrak{D}_{\tilde{x}_{1}} \cap \mathfrak{H}_{1}\right)=\sigma\left(\mathfrak{D}_{\tilde{x}_{1}} \cap \sigma^{-1}\left(\mathfrak{H}_{2}\right)\right)=\mathfrak{D}_{\tilde{x}_{2}} \cap \mathfrak{H}_{2} .
$$

By Proposition 2.1(iii), this implies that $\sharp\left(k\left(x_{1, \mathfrak{H}_{1}}\right)\right)=\sharp\left(k\left(x_{2, \mathfrak{H}_{2}}\right)\right)=\sharp\left(k\left(x_{1, \sigma^{-1}\left(\mathfrak{H}_{2}\right)}\right)\right.$, where $x_{2, \mathfrak{H}_{2}}$ denotes the image of $\tilde{x}_{2}$ in $\Sigma_{X_{2, \mathfrak{H}_{2}}}$. This contradicts

$$
k\left(x_{1, \mathfrak{H}_{1}}\right) \supsetneq k\left(x_{1, \sigma^{-1}\left(\mathfrak{H}_{2}\right)}\right) .
$$

We treat the special case where $\sigma: \mathfrak{G}_{1} \rightarrow \mathfrak{G}_{2}$ is strictly rigid (and hence, in particular, surjective).

Lemma 3.8. Assume that $\sigma: \mathfrak{G}_{1} \rightarrow \mathfrak{G}_{2}$ is strictly rigid.

(i) We have $g_{1}=g_{2}$.

(ii) The map $\phi: \Sigma_{X_{1}} \rightarrow \Sigma_{X_{2}}$ is bijective.

Proof. By Lemma 2.6, the homomorphism $\sigma$ naturally induces a commutative diagram

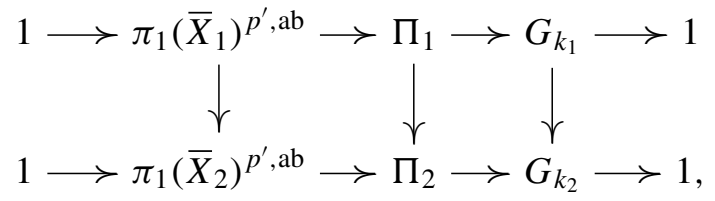

where $\Pi_{i}$ is the quotient $\pi_{1}\left(X_{i}\right)^{\left(p^{\prime}\right)} / \operatorname{Ker}\left(\pi_{1}\left(\bar{X}_{i}\right)^{p^{\prime}} \rightarrow \pi_{1}\left(\bar{X}_{i}\right)^{p^{\prime}, \text { ab }}\right)$, and the maps $\Pi_{i} \rightarrow G_{k_{i}}$ are the natural projections; $i=1$, 2. The vertical maps are surjective. In particular, the representation $G_{k_{1}} \rightarrow G_{k_{2}} \rightarrow \operatorname{Aut}\left(\pi_{1}\left(\bar{X}_{2}\right)^{p^{\prime} \text {,ab }}\right)$, where $G_{k_{2}} \rightarrow \operatorname{Aut}\left(\pi_{1}\left(\bar{X}_{2}\right)^{p^{\prime}, \text { ab }}\right)$ is the natural representation and $G_{k_{1}} \rightarrow G_{k_{2}}$ is the right vertical map in the above diagram, is a quotient representation of the natural representation $G_{k_{1}} \rightarrow \operatorname{Aut}\left(\pi_{1}\left(\bar{X}_{1}\right)^{p^{\prime}, \text { ab }}\right)$. For $i \in\{1,2\}$, let $E_{i}$ be the set of eigenvalues, counted with multiplicities, of the Frobenius element $\varphi_{k_{i}}$ acting on $\pi_{1}\left(\bar{X}_{i}\right)^{p^{\prime}, \text { ab }}$. Then $E_{2} \subset E_{1}$, since the map $G_{k_{1}} \rightarrow G_{k_{2}}$ maps $\varphi_{k_{1}}$ to $\varphi_{k_{2}}$ (see Lemma 2.8(ii)). We will show that $E_{1}=E_{2}$.

For an integer $n \geq 1$, let $k_{i, n}$ be the unique extension of $k_{i}$ of degree $n ; i=1,2$. Then, by the Lefschetz trace formula, $\sharp X_{i}\left(k_{i, n}\right)=1-\sum_{\alpha_{i} \in E_{i}} \alpha_{i}^{n}+q^{n}$, where $q \stackrel{\text { def }}{=} \sharp\left(k_{i}\right)$ (see Lemma 2.8(ii) for the equality $\sharp\left(k_{1}\right)=\sharp\left(k_{2}\right)$ ). Recall that the 
map $\phi: \Sigma_{X_{1}} \rightarrow \Sigma_{X_{2}}$ is surjective (see Lemma 2.9), and if $x_{2}=\phi\left(x_{1}\right)$, then the residue fields $k\left(x_{1}\right)$ and $k\left(x_{2}\right)$ have the same cardinality (see Proposition 2.1(iii)). In particular, $\sharp\left(X_{1}\left(k_{1, n}\right)\right) \geq \sharp\left(X_{2}\left(k_{2, n}\right)\right)$ for all $n$. Thus, $\sum_{j=1}^{r} \beta_{j}^{n} \leq 0$ for any $n \geq 1$, where

$$
E \stackrel{\text { def }}{=} E_{1} \backslash E_{2} \stackrel{\text { def }}{=}\left\{\beta_{1}, \ldots, \beta_{r}\right\}
$$

$\left(r=2 g_{1}-2 g_{2} \geq 0\right)$. Write $\beta_{j}=\rho_{j} e^{i \theta_{j}}\left(\rho_{j} \in \mathbb{R}_{>0}, \theta_{j} \in[0,2 \pi)\right)$, for $j \in\{1, \ldots, r\}$ (note that $\rho_{j}=q^{1 / 2}$ by the Riemann hypothesis for curves). Let $\mathscr{T}$ be the set consisting of the 4 quadrants of $\mathbb{C}=\mathbb{R}^{2}$. More precisely, $\mathscr{T}=\left\{T_{k} \mid k \in\{1,2,3,4\}\right.$, where

$$
T_{k} \stackrel{\text { def }}{=}\left\{\rho e^{i \theta} \mid \rho \in \mathbb{R}_{>0}, \theta \in\left[\frac{(k-1) \pi}{2}, \frac{k \pi}{2}\right)\right\} .
$$

Thus, each $\alpha \in \mathbb{C}^{\times}$belongs to a unique element of $\mathscr{T}$, which we shall denote by $T(\alpha)$. Consider the map $\mu: \mathbb{N} \rightarrow \mathscr{T}^{r}$ that maps an integer $n$ to $\left\{T\left(\beta_{j}^{n}\right)\right\}_{j=1}^{r}$. Then there must exist integers $m_{1}<m_{2}$ such that $\mu\left(m_{1}\right)=\mu\left(m_{2}\right)$, since $\sharp\left(\mathscr{T}^{r}\right)=4^{r}$ is finite. This implies that $e^{i m_{1} \theta_{j}}$ and $e^{i m_{2} \theta_{j}}$ belong to the same quadrant of $\mathbb{C}=\mathbb{R}^{2}$ for all $j \in\{1, \ldots, r\}$. In particular, $\operatorname{Re}\left(\beta_{j}^{n}\right)=\rho_{j}^{n} \cos n \theta_{j}>0$, where $n \stackrel{\text { def }}{=} m_{2}-m_{1} \geq 1$. Suppose that $r>0$; then this implies that

$$
\operatorname{Re}\left(\sum_{j=1}^{r} \beta_{j}^{n}\right)=\sum_{j=1}^{r} \operatorname{Re} \beta_{j}^{n}>0,
$$

which contradicts the above fact that $\sum_{j=1}^{r} \beta_{j}^{n} \leq 0$, for all $n$. Thus, $r=0$, that is, $E=E_{1} \backslash E_{2}$ must be empty, and $E_{1}=E_{2}$.

In particular, the $\hat{\mathbb{Z}}^{p^{\prime}}$-ranks of $\pi_{1}\left(\bar{X}_{i}\right)^{p^{\prime} \text {,ab }}$, which equal $2 g_{i}$, are equal; $i=1,2$. This completes the proof of Lemma 3.8(i).

Finally, we can conclude that $\phi$ is injective. For otherwise, there would exist an integer $n \geq 1$ such that $\sharp\left(X_{1}\left(k_{n}\right)\right)>\sharp\left(X_{2}\left(k_{n}\right)\right)$, and hence, $E \neq \varnothing$, which is a contradiction. This completes the proof of Lemma 3.8(ii).

Lemma 3.9. Assume that $\sigma: \mathfrak{G}_{1} \rightarrow \mathfrak{G}_{2}$ is strictly rigid. Then $\sigma^{\left(p^{\prime}\right)}: \mathfrak{G}_{1}^{\left(p^{\prime}\right)} \rightarrow \mathfrak{G}_{2}^{\left(p^{\prime}\right)}$ is an isomorphism. (In particular, in the prime-to-characteristic case, $\sigma$ is an isomorphism.)

Proof. By Lemma 3.8(ii), the map $\phi: \Sigma_{X_{1}} \rightarrow \Sigma_{X_{2}}$ induced by $\sigma$ is bijective. For a finite subset $S_{2}$ of $\Sigma_{X_{2}}$, let $S_{1} \stackrel{\text { def }}{=} \phi^{-1}\left(S_{2}\right)$. Then $\sigma$ naturally induces a continuous, surjective homomorphism $\tau_{S_{1}, S_{2}}^{\prime}: \pi_{1}\left(U_{1}\right)^{\left(p^{\prime}\right)} \rightarrow \pi_{1}\left(U_{2}\right)^{\left(p^{\prime}\right)}$, where $\pi_{1}\left(U_{i}\right)^{\left(p^{\prime}\right)}$ is the maximal geometrically prime-to- $p$ quotient of the fundamental group $\pi_{1}\left(U_{i}\right)$ of $U_{i} \stackrel{\text { def }}{=} X_{i}-S_{i} ; i=1,2$. Further, we have the commutative diagram

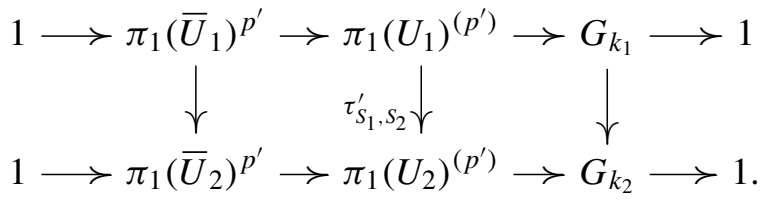


The surjective homomorphism $\pi_{1}\left(\bar{U}_{1}\right)^{p^{\prime}} \rightarrow \pi_{1}\left(\bar{U}_{2}\right)^{p^{\prime}}$ must be an isomorphism by [Fried and Jarden 1986, Proposition 15.4], since $X_{i}-S_{i}$ have the same topological type $\left(g_{i}, \sharp\left(\bar{S}_{i}\right)\right)$, where $\bar{S}_{i}$ denotes the inverse image of $S_{i}$ in $\Sigma_{\bar{X}_{i}} ; i=1,2$, by Lemma 3.8. (For the bijectivity $\bar{S}_{1} \stackrel{\sim}{\rightarrow} \bar{S}_{2}$, apply Lemma 3.8(ii) to various open subgroups of $\mathfrak{G}_{1}, \mathfrak{G}_{2}$ corresponding to constant field extensions.) Thus, the map $\tau_{S_{1}, S_{2}}^{\prime}$ is an isomorphism (note that the surjective map $G_{k_{1}} \rightarrow G_{k_{2}}$ is an isomorphism). Also,

$$
\mathfrak{G}_{i}^{\left(p^{\prime}\right)}=\underset{S_{i}}{\lim } \pi_{1}\left(X_{i}-S_{i}\right)^{\left(p^{\prime}\right)}
$$

where the projective limit is taken over all finite subsets $S_{i}$ of $\Sigma_{X_{i}} ; i=1,2$. Further,

$$
\sigma^{\left(p^{\prime}\right)}=\lim _{\left\{S_{1}, S_{2}\right\}} \tau_{S_{1}, S_{2}}^{\prime}
$$

where the projective limit is taken over all finite subsets $S_{1}$ and $S_{2}$ corresponding to each other via $\phi$. Thus, $\sigma^{\left(p^{\prime}\right)}$ must be an isomorphism.

Now, return to the general case. As above, let $\mathfrak{H}_{1} \subset \mathfrak{G}_{1}, \mathfrak{H}_{2} \subset \mathfrak{G}_{2}$ be open subgroups such that $\sigma\left(\mathfrak{H}_{1}\right) \subset \mathfrak{H}_{2}$ and the map $\sigma_{\mathfrak{H}_{1}, \mathfrak{H}_{2}}: \mathfrak{H}_{1} \rightarrow \mathfrak{H}_{2}$ obtained by restricting $\sigma: \mathfrak{G}_{1} \rightarrow \mathfrak{G}_{2}$ is strictly rigid with respect to $\tilde{\phi}: \Sigma_{\tilde{X}_{1}} \rightarrow \Sigma_{\tilde{X}_{2}}$. By Remark 3.2(iv), $\tilde{\phi}$ is Galois-equivariant with respect to $\sigma: \mathfrak{G}_{1} \rightarrow \mathfrak{G}_{2}$ (that is, not only with respect to $\sigma_{\mathfrak{H}_{1}, \mathfrak{H}_{2}}: \mathfrak{H}_{1} \rightarrow \mathfrak{H}_{2}$ ), and, for each $\tilde{x}_{1} \in \Sigma_{\tilde{X}_{1}}$, we have

$$
\sigma\left(\mathfrak{D}_{\tilde{x}_{1}}\right) \underset{\text { open }}{\subset} \mathfrak{D}_{\tilde{\phi}\left(\tilde{x}_{1}\right)}, \quad \sigma\left(\mathfrak{D}_{\tilde{x}_{1}} \cap \mathfrak{H}_{1}\right)=\mathfrak{D}_{\tilde{\phi}\left(\tilde{x}_{1}\right)} \cap \mathfrak{H}_{2} .
$$

Moreover, by Lemma 3.9, the map $\left(\sigma_{\mathfrak{H}_{1}, \mathfrak{H}_{2}}\right)^{\left(p^{\prime}\right)}: \mathfrak{H}_{1}^{\left(p^{\prime}\right)} \rightarrow \mathfrak{H}_{2}^{\left(p^{\prime}\right)}$ induced by $\sigma_{\mathfrak{H}_{1}, \mathfrak{H}_{2}}$ is an isomorphism.

Now, let us denote the finite separable extension of $K_{i}$ corresponding to $\mathfrak{H}_{i} \subset \mathfrak{G}_{i}$ by $K_{i, \mathfrak{H}_{i}}$, and the (infinite) Galois extension of $K_{i, \mathfrak{H}_{i}}$ corresponding to $\mathfrak{H}_{i} \rightarrow \mathfrak{H}_{i}^{\left(p^{\prime}\right)}$ by $\tilde{K}_{i, \mathfrak{H}_{i}}^{\left(p^{\prime}\right)}$. By applying the Isom-form proved in [Saïdi and Tamagawa 2009], we see that

$$
\left(\sigma_{\mathfrak{H}_{1}, \mathfrak{H}_{2}}\right)^{\left(p^{\prime}\right)}: \mathfrak{H}_{1}^{\left(p^{\prime}\right)} \stackrel{\sim}{\rightarrow} \mathfrak{H}_{2}^{\left(p^{\prime}\right)}
$$

arises from a unique field isomorphism $\gamma_{\mathfrak{H}_{1}^{\left(p^{\prime}\right)}, \mathfrak{H}_{2}^{\left(p^{\prime}\right)}}: \tilde{K}_{2, \mathfrak{H}_{2}}^{\left(p^{\prime}\right)} \stackrel{\sim}{\rightarrow} \tilde{K}_{1, \mathfrak{H}_{1}}^{\left(p^{\prime}\right)}$ that induces an isomorphism $K_{2, \mathfrak{H}_{2}} \stackrel{\sim}{\rightarrow} K_{1, \mathfrak{H}_{1}}$.

Lemma 3.10. Let $\mathfrak{H}_{i}^{\prime} \subset \mathfrak{H}_{i}, i=1,2$ be open subgroups, such that $\sigma\left(\mathfrak{H}_{1}^{\prime}\right) \subset \mathfrak{H}_{2}^{\prime}$ and that $\sigma_{\mathfrak{H}_{1}^{\prime}, \mathfrak{H}_{2}^{\prime}}: \mathfrak{H}_{1}^{\prime} \rightarrow \mathfrak{H}_{2}^{\prime}$ is strictly rigid. Then the field isomorphism

$$
\gamma_{\left.\left(\mathfrak{H}_{1}^{\prime}\right)^{\left(p^{\prime}\right),\left(\mathfrak{H}_{2}^{\prime}\right.}\right)^{\left(p^{\prime}\right)}}: \tilde{K}_{2, \mathfrak{H}_{2}^{\prime}}^{\left(p^{\prime}\right)} \stackrel{\sim}{\rightarrow} \tilde{K}_{1, \mathfrak{H}_{1}^{\prime}}^{\left(p^{\prime}\right)}
$$

restricts to $\gamma_{\mathfrak{H}_{1}^{\left(p^{\prime}\right)}, \mathfrak{H}_{2}^{\left(p^{\prime}\right)}}: \tilde{K}_{2, \mathfrak{H}_{2}}^{\left(p^{\prime}\right)} \stackrel{\sim}{\rightarrow} \tilde{K}_{1, \mathfrak{H}_{1}}^{\left(p^{\prime}\right)}$ 
Proof. This follows formally from the statement of the Isom-form proved in [Saïdi and Tamagawa 2009], as follows, without recalling any construction in that paper.

Take an open subgroup $\mathfrak{H}_{2}^{\prime \prime}$ of $\mathfrak{H}_{2}^{\prime}$ that is normal in $\mathfrak{H}_{2}$. Then, by Lemma 3.7,

$$
\mathfrak{H}_{1}^{\prime \prime} \stackrel{\text { def }}{=} \sigma^{-1}\left(\mathfrak{H}_{2}^{\prime \prime}\right) \subset \sigma^{-1}\left(\mathfrak{H}_{2}^{\prime}\right)=\mathfrak{H}_{1}^{\prime} \text {, }
$$

and hence, by Remark 3.2(ii), $\mathfrak{H}_{1}^{\prime \prime} \stackrel{\sigma}{\rightarrow} \mathfrak{H}_{2}^{\prime \prime}$ is strictly rigid. Assume that

$$
\gamma_{\left(\mathfrak{H}_{1}^{\prime \prime}\right)\left(p^{\prime}\right),\left(\mathfrak{H}_{2}^{\prime \prime}\right)\left(p^{\prime}\right)}: \tilde{K}_{2, \mathfrak{H}_{2}^{\prime \prime}}^{\left(p^{\prime}\right)} \stackrel{\sim}{\rightarrow} \tilde{K}_{1, \mathfrak{H}_{1}^{\prime \prime}}^{\left(p^{\prime}\right)}
$$

restricts to $\gamma_{\left(\mathfrak{H}_{1}^{\prime}\right)\left(p^{\prime}\right),\left(\mathfrak{H}_{2}^{\prime}\right)^{\left(p^{\prime}\right)}}: \tilde{K}_{2, \mathfrak{H}_{2}^{\prime}}^{\left(p^{\prime}\right)} \stackrel{\sim}{\rightarrow} \tilde{K}_{1, \mathfrak{H}_{1}^{\prime}}^{\left(p^{\prime}\right)}$ and to $\gamma_{\mathfrak{H}_{1}^{\left(p^{\prime}\right)}, \mathfrak{H}_{2}^{\left(p^{\prime}\right)}}: \tilde{K}_{2, \mathfrak{H}_{2}}^{\left(p^{\prime}\right)} \stackrel{\sim}{\rightarrow} \tilde{K}_{1, \mathfrak{H}_{1}}^{\left(p^{\prime}\right)}$. Then

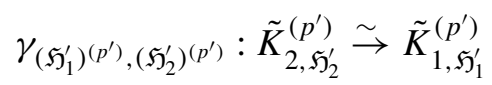

restricts to

$$
\gamma_{\mathfrak{H}_{1}^{\left(p^{\prime}\right)}, \mathfrak{H}_{2}^{\left(p^{\prime}\right)}}: \tilde{K}_{2, \mathfrak{H}_{2}}^{\left(p^{\prime}\right)} \stackrel{\sim}{\rightarrow} \tilde{K}_{1, \mathfrak{H}_{1}}^{\left(p^{\prime}\right)},
$$

as desired. So, it suffices to prove the desired property in the case where $\mathfrak{H}_{i}^{\prime} \subset \mathfrak{H}_{i}$ is normal for $i=1,2$, and $\sigma$ naturally induces an isomorphism $\mathfrak{H}_{1} / \mathfrak{H}_{1}^{\prime} \stackrel{\sim}{\rightarrow} \mathfrak{H}_{2} / \mathfrak{H}_{2}^{\prime}$ between finite groups.

For $i=1,2$, let $\mathfrak{J}_{i}^{\prime}$ be the image of $\mathfrak{H}_{i}^{\prime}$ in $\mathfrak{H}_{i}^{\left(p^{\prime}\right)}$, which is an open normal subgroup of $\mathfrak{H}_{i}^{\left(p^{\prime}\right)}$. Let $\mathfrak{J}_{i} \subset \mathfrak{H}_{i}$ be the inverse image of $\mathfrak{J}_{i}^{\prime}$ in $\mathfrak{H}_{i}$. Thus, we have the natural identification $\mathfrak{J}_{i}^{\left(p^{\prime}\right)}=\mathfrak{J}_{i}^{\prime}$ and the commutative diagram

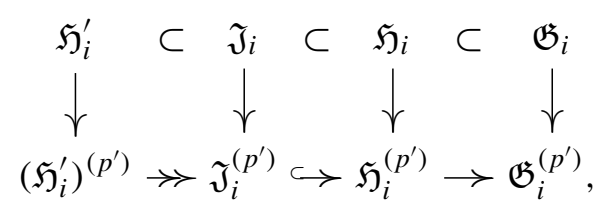

in which the vertical arrows are natural surjective maps.

Since the isomorphism $\left(\sigma_{\mathfrak{H}_{1}^{\prime}, \mathfrak{H}_{2}^{\prime}}\right)^{\left(p^{\prime}\right)}:\left(\mathfrak{H}_{1}^{\prime}\right)^{\left(p^{\prime}\right)} \stackrel{\sim}{\rightarrow}\left(\mathfrak{H}_{2}^{\prime}\right)^{\left(p^{\prime}\right)}$ is compatible with the natural (conjugate) actions of $\mathfrak{H}_{1}$ and $\mathfrak{H}_{2}$ with respect to $\mathfrak{H}_{1} \stackrel{\sigma}{\rightarrow} \mathfrak{H}_{2}$, the corresponding field isomorphism

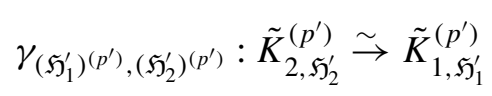

is also compatible with the natural actions of $\mathfrak{H}_{1}$ and $\mathfrak{H}_{2}$ with respect to $\mathfrak{H}_{1} \stackrel{\sigma}{\rightarrow} \mathfrak{H}_{2}$. In particular, $\left.\gamma_{\left(\mathfrak{H}_{1}^{\prime}\right)}\right)^{\left(p^{\prime}\right)},\left(\mathfrak{H}_{2}^{\prime}\right)^{\left(p^{\prime}\right)}$ restricts to $K_{2, \mathfrak{H}_{2}} \stackrel{\sim}{\rightarrow} K_{1, \mathfrak{H}_{1}}$, and hence induces an isomorphism

$$
\alpha: K_{2, \mathfrak{H}_{2}}^{\left(p^{\prime}\right)} \stackrel{\sim}{\rightarrow} K_{1, \mathfrak{H}_{1}}^{\left(p^{\prime}\right)}
$$

that is compatible with $\sigma: \mathfrak{H}_{1} \rightarrow \mathfrak{H}_{2}$, and hence with $\sigma_{\mathfrak{H}_{1}^{\left(p^{\prime}\right)}, \mathfrak{H}_{2}^{\left(p^{\prime}\right)}}: \mathfrak{H}_{1}^{\left(p^{\prime}\right)} \stackrel{\sim}{\rightarrow} \mathfrak{H}_{2}^{\left(p^{\prime}\right)}$. On the other hand, the isomorphism

$$
\gamma_{\mathfrak{H}_{1}^{\left(p^{\prime}\right)}, \mathfrak{H}_{2}^{\left(p^{\prime}\right)}}: \tilde{K}_{2, \mathfrak{H}_{2}}^{\left(p^{\prime}\right)} \stackrel{\sim}{\rightarrow} \tilde{K}_{1, \mathfrak{H}_{1}}^{\left(p^{\prime}\right)}
$$


is also compatible with

$$
\sigma_{\mathfrak{H}_{1}^{\left(p^{\prime}\right)}, \mathfrak{H}_{2}^{\left(p^{\prime}\right)}}: \mathfrak{H}_{1}^{\left(p^{\prime}\right)} \stackrel{\sim}{\rightarrow} \mathfrak{H}_{2}^{\left(p^{\prime}\right)} .
$$

Thus, we conclude that, as desired,

$$
\alpha=\sigma_{\mathfrak{H}_{1}^{\left(p^{\prime}\right)}, \mathfrak{H}_{2}^{\left(p^{\prime}\right)}},
$$

by the uniqueness of such a Galois-compatible isomorphism. (This is included in the statement of the Isom-form proved in [Saïdi and Tamagawa 2009].)

Now, consider the set $\mathscr{Y}\left(\subset \operatorname{Sub}\left(\mathfrak{G}_{1}\right) \times \operatorname{Sub}\left(\mathfrak{G}_{2}\right)\right)$ of all pairs of open subgroups $\mathfrak{H}_{1} \subset \mathfrak{G}_{1}, \mathfrak{H}_{2} \subset \mathfrak{G}_{2}$ such that $\sigma\left(\mathfrak{H}_{1}\right) \subset \mathfrak{H}_{2}$, that $\mathfrak{H}_{1} \stackrel{\sigma}{\rightarrow} \mathfrak{H}_{2}$ is strictly rigid, and that $\mathfrak{H}_{2}$ is normal in $\mathfrak{G}_{2}$. Then, as in the proof of Lemma 3.10, it follows from Lemma 3.7 and Remark 3.2(ii) that $\left(\mathfrak{H}_{1}, \mathfrak{H}_{2}\right) \in \mathscr{Y}$ implies that $\sigma\left(\mathfrak{H}_{1}\right)=\mathfrak{H}_{2}$, that $\mathfrak{H}_{1}=\sigma^{-1}\left(\mathfrak{H}_{2}\right)$, and that the image of $\mathscr{Y}$ in $\operatorname{Sub}\left(\mathfrak{G}_{2}\right)$ is cofinal in the set of open subgroups of $\mathfrak{G}_{2}$.

For each pair $\left(\mathfrak{H}_{1}, \mathfrak{H}_{2}\right) \in \mathscr{T}$, we get an isomorphism

$$
\sigma_{\mathfrak{H}_{1}^{\left(p^{\prime}\right)}, \mathfrak{H}_{2}^{\left(p^{\prime}\right)}}: \mathfrak{H}_{1}^{\left(p^{\prime}\right)} \stackrel{\sim}{\rightarrow} \mathfrak{H}_{2}^{\left(p^{\prime}\right)}
$$

by Lemma 3.9, which is Galois-compatible with respect to $\sigma: \mathfrak{G}_{1} \rightarrow \mathfrak{G}_{2}$. By the Isom-form proved in [Saïdi and Tamagawa 2009], $\sigma_{\mathfrak{H}_{1}^{\left(p^{\prime}\right)}, \mathfrak{H}_{2}^{\left(p^{\prime}\right)}}$ induces an isomorphism

$$
\gamma_{\mathfrak{H}_{1}^{\left(p^{\prime}\right)}, \mathfrak{H}_{2}^{\left(p^{\prime}\right)}}: \tilde{K}_{2, \mathfrak{H}_{2}}^{\left(p^{\prime}\right)} \stackrel{\sim}{\rightarrow} \tilde{K}_{1, \mathfrak{H}_{1}}^{\left(p^{\prime}\right)},
$$

which is Galois-compatible with respect to $\sigma: \mathfrak{G}_{1} \rightarrow \mathfrak{G}_{2}$. By Lemma 3.10,

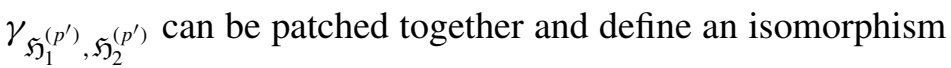

$$
\tilde{\gamma}: \tilde{K}_{2} \stackrel{\sim}{\rightarrow}\left(\tilde{K}_{1}\right)^{\mathfrak{N}},
$$

where

$$
\mathfrak{N} \stackrel{\text { def }}{=} \operatorname{Ker}\left(\sigma: \mathfrak{G}_{1} \rightarrow \mathfrak{G}_{2}\right),
$$

which is Galois-compatible with respect to $\sigma: \mathfrak{G}_{1} \rightarrow \mathfrak{G}_{2}$.

In the profinite (resp. prime-to-characteristic) case, $\tilde{K}_{2}$ admits no nontrivial finite separable (resp. geometrically prime-to- $p$ ) extension, and hence neither does $\left(\tilde{K}_{1}\right)^{\mathfrak{N}}\left(\simeq \tilde{K}_{2}\right)$. This implies that $\left(\tilde{K}_{1}\right)^{\mathfrak{N}}=\tilde{K}_{1}$, that is, $\mathfrak{N}=\{1\}$. Thus, we obtain $\tilde{\gamma}: \tilde{K}_{2} \stackrel{\sim}{\rightarrow} \tilde{K}_{1}$, which is Galois-compatible with respect to $\sigma: \mathfrak{G}_{1} \rightarrow \mathfrak{G}_{2}$, as desired. Finally, the uniqueness of such $\tilde{\gamma}$ follows formally from the uniqueness in the statement of the Isom-form, proved in [Uchida 1977; Saïdi and Tamagawa 2009]. This finishes the proof of Theorem 3.4.

Remark 3.11. We have proved Theorem 3.4 by reducing it to the statement of the Isom-form, by means of Lemma 3.9. Instead, we could mimic the proof of the Isom-form. 


\section{Proper homomorphisms between Galois groups}

In this section we investigate a class of homomorphisms between (geometrically prime-to-characteristic quotients of) absolute Galois groups of function fields of curves over finite fields, which we call proper. We follow the notations in Sections $1-3$, and in particular, the Notation at the beginning of subsection $2 \mathrm{~B}$. We assume that Condition 3 holds.

Definition 4.1 (well-behaved homomorphisms). We say that $\sigma: \mathfrak{G}_{1} \rightarrow \mathfrak{G}_{2}$ is wellbehaved if there exists a map

$$
\tilde{\phi}: \Sigma_{\tilde{X}_{1}} \rightarrow \Sigma_{\tilde{X}_{2}}
$$

such that $\sigma\left(\mathfrak{D}_{\tilde{x}_{1}}\right) \underset{\text { open }}{\subset} \mathfrak{D}_{\tilde{\phi}\left(\tilde{x}_{1}\right)}$ for each $\tilde{x}_{1} \in \Sigma_{\tilde{X}_{1}}$.

Remark 4.2. (i) Given a commutative diagram of maps between profinite groups

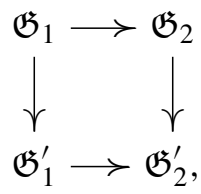

where the vertical arrows are surjective and the map $\mathfrak{G}_{1} \rightarrow \mathfrak{G}_{2}$ is well-behaved, then the map $\mathfrak{G}_{1}^{\prime} \rightarrow \mathfrak{G}_{2}^{\prime}$ is well-behaved.

(ii) Let $\mathfrak{H}_{1} \subset \mathfrak{G}_{1}, \mathfrak{H}_{2} \subset \mathfrak{G}_{2}$ be open subgroups such that $\sigma\left(\mathfrak{H}_{1}\right) \subset \mathfrak{H}_{2}$. Then if $\sigma: \mathfrak{G}_{1} \rightarrow \mathfrak{G}_{2}$ is well-behaved, the natural homomorphism $\mathfrak{H}_{1} \rightarrow \mathfrak{H}_{2}$ induced by $\sigma$ is well-behaved. (Here, $\mathfrak{H}_{i}$ is considered as a quotient of the absolute Galois group that is the inverse image in $G_{i}$ of $\mathfrak{H}_{i} \subset \mathfrak{G}_{i}$.)

(iii) If $\sigma: \mathfrak{G}_{1} \rightarrow \mathfrak{G}_{2}$ is strictly rigid (Definition 3.1), then it is well-behaved.

(iv) As in Proposition 2.2, let $l \neq p_{1}, p_{2}$ be a prime number, and assume that (1) $N_{2}^{l}=N_{2}$, or, equivalently, $\tilde{K}_{2}$ admits no $l$-cyclic extension; and (2) $\tilde{K}_{2}$ contains a primitive $l$-th roots of unity. Then, first, if $\sigma: \mathfrak{G}_{1} \rightarrow \mathfrak{G}_{2}$ is rigid, then it is wellbehaved by Remark 3.2(iv). Second, if $\sigma: \mathfrak{G}_{1} \rightarrow \mathfrak{G}_{2}$ is well-behaved with respect to $\tilde{\phi}: \Sigma_{\tilde{X}_{1}} \rightarrow \Sigma_{\tilde{X}_{2}}$, then we must have

$$
\Sigma_{\tilde{X}_{1}, \sigma, l}=\Sigma_{\tilde{X}_{1}} \quad \text { and } \quad \tilde{\phi}=\tilde{\phi}_{\sigma, l} .
$$

In particular, then $\tilde{\phi}$ is unique and Galois-equivariant with respect to $\sigma$, and hence naturally induces a map $\phi\left(=\phi_{\sigma, l}\right): \Sigma_{X_{1}} \rightarrow \Sigma_{X_{2}}$.

Definition 4.3 (proper homomorphisms). We say that $\sigma: \mathfrak{G}_{1} \rightarrow \mathfrak{G}_{2}$ is proper if $\sigma$ is well-behaved with respect to $\tilde{\phi}: \Sigma_{\tilde{X}_{1}} \rightarrow \Sigma_{\tilde{X}_{2}}$, such that $\tilde{\phi}$ is Galois-equivariant with respect to $\sigma$, and the map $\phi: \Sigma_{X_{1}} \rightarrow \Sigma_{X_{2}}$ induced by $\tilde{\phi}$ has finite fibers, that is, for each $x_{2} \in \Sigma_{X_{2}}$, the fiber $\phi^{-1}\left(x_{2}\right)$ is a (possibly empty) finite set. 
Remark 4.4. (i) Given a commutative diagram of maps between profinite groups

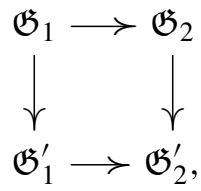

where the vertical arrows are surjective and the map $\mathfrak{G}_{1} \rightarrow \mathfrak{G}_{2}$ is proper, the map $\mathfrak{G}_{1}^{\prime} \rightarrow \mathfrak{G}_{2}^{\prime}$ is proper.

(ii) Let $\mathfrak{H}_{1} \subset \mathfrak{G}_{1}, \mathfrak{H}_{2} \subset \mathfrak{G}_{2}$ be open subgroups such that $\sigma\left(\mathfrak{H}_{1}\right) \subset \mathfrak{H}_{2}$. Then if $\sigma: \mathfrak{G}_{1} \rightarrow \mathfrak{G}_{2}$ is proper, the natural homomorphism $\mathfrak{H}_{1} \rightarrow \mathfrak{H}_{2}$ induced by $\sigma$ is proper. (Here, $\mathfrak{H}_{i}$ is considered as a quotient of the absolute Galois group that is the inverse image in $G_{i}$ of $\mathfrak{H}_{i} \subset \mathfrak{G}_{i}$.)

In the rest of this section, we assume that Condition 1 holds. Assume also that the continuous open homomorphism $\sigma: \mathfrak{G}_{1} \rightarrow \mathfrak{G}_{2}$ is well-behaved with respect to $\tilde{\phi}: \Sigma_{\tilde{X}_{1}} \rightarrow \Sigma_{\tilde{X}_{2}}$. By Lemma 2.4, we have $p \stackrel{\text { def }}{=} p_{1}=p_{2}$. Let $\tilde{x}_{1} \in \Sigma_{\tilde{X}_{1}}$ and set $\tilde{x}_{2} \stackrel{\text { def }}{=} \tilde{\phi}\left(\tilde{x}_{1}\right)$. Denote by $x_{1}$ and $x_{2}$ the image of $\tilde{x}_{1}$ and $\tilde{x}_{2}$ in $\Sigma_{X_{1}}$ and $\Sigma_{X_{2}}$, respectively. Then

$$
\mathfrak{D}_{\tilde{x}_{1}} \stackrel{\sigma}{\rightarrow} \sigma\left(\mathfrak{D}_{\tilde{x}_{1}}\right) \underset{\text { open }}{\subset} \mathfrak{D}_{\tilde{x}_{2}} .
$$

By this and Proposition 2.1(v), we have

$$
\mathfrak{I}_{\tilde{x}_{1}} \stackrel{\sigma}{\rightarrow} \sigma\left(\mathfrak{I}_{\tilde{x}_{1}}\right) \underset{\text { open }}{\subset} \mathfrak{I}_{\tilde{x}_{2}} .
$$

In particular, $\sigma$ induces an open injective homomorphism $\tau_{\tilde{x}_{1}}^{\mathrm{t}}: \mathfrak{I}_{\tilde{x}_{1}}^{\mathrm{t}} \hookrightarrow \mathfrak{I}_{\tilde{x}_{2}}^{\mathrm{t}}$, where $\mathfrak{I}_{\tilde{x}_{1}}^{\mathrm{t}}\left(\right.$ resp. $\mathfrak{I}_{\tilde{x}_{2}}^{\mathrm{t}}$ ) denotes the inertia subgroup of $\mathfrak{D}_{\tilde{x}_{1}}^{\mathrm{t}}$ (resp. of $\mathfrak{D}_{\tilde{x}_{2}}^{\mathrm{t}}$ ). We have natural identifications

$$
M_{1} \stackrel{\sim}{\rightarrow} M_{k\left(x_{1}\right)^{\text {sep }}} \stackrel{\sim}{\rightarrow} \mathfrak{I}_{\tilde{x}_{1}}^{\mathrm{t}}, \quad M_{2} \stackrel{\sim}{\rightarrow} M_{k\left(x_{2}\right)^{\text {sep }}} \stackrel{\sim}{\rightarrow} \mathfrak{I}_{\tilde{x}_{2}}^{\mathrm{t}},
$$

where $M_{i} \stackrel{\text { def }}{=} M_{K_{i}^{\text {sep }}}$ is the (global) module of roots of unity for $i=1,2$.

We introduce the following important concept of rigidity of inertia.

Definition 4.5 (inertia-rigid homomorphisms). We say that the well-behaved homomorphism $\sigma: \mathfrak{G}_{1} \rightarrow \mathfrak{G}_{2}$ is inertia-rigid if there exists an isomorphism

$$
\tau: M_{1} \stackrel{\sim}{\rightarrow} M_{2}
$$

of $\hat{\mathbb{Z}}^{p^{\prime}}$-modules such that for each $\tilde{x}_{1} \in \Sigma_{\tilde{X}_{1}}$, there exists a positive integer $e_{\tilde{x}_{1}}$ such that the following diagram commutes:

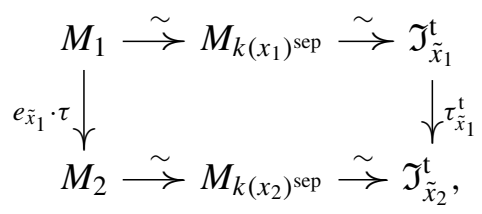


where $\tilde{x}_{2} \stackrel{\text { def }}{=} \tilde{\phi}\left(\tilde{x}_{1}\right) ; x_{1}$ and $x_{2}$ are the images of $\tilde{x}_{1}$ and $\tilde{x}_{2}$ in $\Sigma_{X_{1}}$ and $\Sigma_{X_{2}}$, respectively; and the isomorphisms are the canonical identifications.

Remark 4.6. (i) Given a commutative diagram of maps between profinite groups

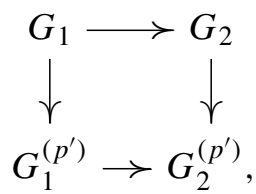

where the vertical arrows are natural surjective maps and the map $G_{1} \rightarrow G_{2}$ is inertia-rigid, the map $G_{1}^{\left(p^{\prime}\right)} \rightarrow G_{2}^{\left(p^{\prime}\right)}$ is inertia-rigid.

(ii) Let $\mathfrak{H}_{1} \subset \mathfrak{G}_{1}, \mathfrak{H}_{2} \subset \mathfrak{G}_{2}$ be open subgroups such that $\sigma\left(\mathfrak{H}_{1}\right) \subset \mathfrak{H}_{2}$. Then if $\sigma: \mathfrak{G}_{1} \rightarrow \mathfrak{G}_{2}$ is inertia-rigid, the natural homomorphism $\mathfrak{H}_{1} \rightarrow \mathfrak{H}_{2}$ induced by $\sigma$ is inertia-rigid. (Here, $\mathfrak{H}_{i}$ is considered as a quotient of the absolute Galois group that is the inverse image in $G_{i}$ of $\mathfrak{H}_{i} \subset \mathfrak{G}_{i}$.)

Remark 4.7. (i) Set

$$
\mathfrak{e}_{\tilde{x}_{1}} \stackrel{\text { def }}{=}\left[\mathfrak{I}_{\tilde{x}_{2}}: \sigma\left(\mathfrak{I}_{\tilde{x}_{1}}\right)\right], \quad \mathfrak{e}_{\tilde{x}_{1}}^{\mathrm{t}} \stackrel{\text { def }}{=}\left[\mathfrak{I}_{\tilde{x}_{2}}^{\mathrm{t}}: \tau_{\tilde{x}_{1}}^{\mathrm{t}}\left(\mathfrak{I}_{\tilde{x}_{1}}^{\mathrm{t}}\right)\right] .
$$

Note that $p \nmid \mathfrak{e}_{\tilde{x}_{1}}^{\mathrm{t}}$ and there exists an integer $b_{\tilde{x}_{1}} \geq 0$ such that $\mathfrak{e}_{\tilde{x}_{1}}=p^{b_{\tilde{x}_{1}}} \mathfrak{e}_{\tilde{x}_{1}}^{\mathrm{t}}$. (In the prime-to-characteristic case, we have $\mathfrak{e}_{\tilde{x}_{1}}=\mathfrak{e}_{\tilde{x}_{1}}^{\mathrm{t}}$ and $b_{\tilde{x}_{1}}=0$.) Now, in Definition 4.5 , there must exist an integer $a_{\tilde{x}_{1}} \geq 0$ such that $e_{\tilde{x}_{1}}=p^{a_{\tilde{x}_{1}}} \mathfrak{e}_{\tilde{x}_{1}}^{\mathrm{t}}$, or, equivalently, $e_{\tilde{x}_{1}}=p^{c_{\tilde{x}_{1}}} \mathfrak{e}_{\tilde{x}_{1}}$, where $c_{\tilde{x}_{1}} \stackrel{\text { def }}{=} a_{\tilde{x}_{1}}-b_{\tilde{x}_{1}} \in \mathbb{Z}$. Moreover, set

$$
a \stackrel{\text { def }}{=} \min \left\{a_{\tilde{x}_{1}} \mid \tilde{x}_{1} \in \Sigma_{\tilde{X}_{1}}\right\} .
$$

Replacing $\tau$ by $p^{a} \tau$ and $e_{\tilde{x}_{1}}$ by $p^{-a} e_{\tilde{x}_{1}}=p^{a_{\tilde{x}_{1}}-a} \mathfrak{e}_{\tilde{x}_{1}}^{\mathrm{t}}$, we may assume that $a=0$.

Assume, moreover, that $\sigma$ is proper and that we are in the profinite case. Then, in fact, we have $c_{\tilde{x}_{1}}=0$ for every $\tilde{x}_{1} \in \tilde{X}_{1}$ eventually, if we choose $\tau$ with $a=0$. (This follows from Theorem 4.8 below and its proof.) Thus, in the profinite case, we may assume $e_{\tilde{x}_{1}}=\mathfrak{e}_{\tilde{x}_{1}}$ in Definition 4.5 from the beginning. In the prime-to-characteristic case, however, it seems difficult to specify the value of $e_{\tilde{x}_{1}}$ a priori. (If we assumed $e_{\tilde{x}_{1}}=\mathfrak{e}_{\tilde{x}_{1}}$ in the prime-to-characteristic case, then inertia-rigid homomorphisms would cover only tame homomorphisms $K_{2} \rightarrow K_{1}$.)

(ii) In the situation of Definition 4.5, we have

$$
\mathfrak{D}_{\tilde{x}_{1}} \stackrel{\sigma}{\rightarrow} \mathfrak{E}_{\tilde{x}_{1}} \stackrel{\text { def }}{=} \sigma\left(\mathfrak{D}_{\tilde{x}_{1}}\right) \subset \mathfrak{D}_{\tilde{x}_{2}} .
$$

The subgroup $\mathfrak{E}_{\tilde{x}_{1}} \subset \mathfrak{D}_{\tilde{x}_{2}}$ corresponds to a finite extension $L_{x_{1}} /\left(K_{2}\right)_{x_{2}}$ of the $x_{2}$-adic completion $\left(K_{2}\right)_{x_{2}}$ of $K_{2}$. Thus, the residue field $\ell_{x_{1}}$ of $L_{x_{1}}$ is a finite extension of 
the residue field $k\left(x_{2}\right)$ at $x_{2}$. We have the commutative diagram

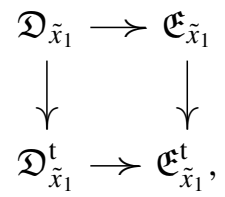

where the vertical maps are the canonical surjections onto the maximal tame quotients, and the horizontal maps are naturally induced by $\sigma$. Further, the lower horizontal map, which is surjective, naturally induces an isomorphism $\mathfrak{I}_{\tilde{x}_{1}}^{\mathrm{t}} \stackrel{\sim}{\rightarrow} \mathfrak{J}_{\tilde{x}_{1}}^{\mathrm{t}}$ by Proposition 2.1(v). Here, $\mathfrak{I}_{\tilde{x}_{1}}^{\mathrm{t}}$ and $\mathfrak{J}_{\tilde{x}_{1}}^{\mathrm{t}}$ denote the inertia subgroups of $\mathfrak{D}_{\tilde{x}_{1}}^{\mathrm{t}}$ and $\mathfrak{E}_{\tilde{x}_{1}}^{\mathrm{t}}$, respectively. We have a natural identification $\mathfrak{J}_{\tilde{x}_{1}}^{\mathrm{t}} \stackrel{\sim}{\rightarrow} \mathfrak{I}_{\tilde{x}_{2}}^{\mathrm{t}}$, where $\mathfrak{I}_{\tilde{x}_{2}}^{\mathrm{t}}$ is the inertia subgroup of $\mathfrak{D}_{\tilde{x}_{2}}^{\mathrm{t}}$, obtained via the natural identifications

$$
M_{\left(K_{2}\right)_{x_{2}}^{\text {sep }}} \stackrel{\sim}{\rightarrow} \mathfrak{I}_{\tilde{x}_{2}}^{\mathrm{t}}, \quad M_{L_{x_{1}}^{\text {sep }}} \stackrel{\sim}{\rightarrow} \mathfrak{J}_{\tilde{x}_{1}}^{\mathrm{t}}, \quad\left(K_{2}\right)_{x_{2}}^{\mathrm{sep}}=L_{x_{1}}^{\mathrm{sep}},
$$

which, composed with the natural map $\mathfrak{J}_{\tilde{x}_{1}}^{\mathrm{t}} \rightarrow \mathfrak{I}_{\tilde{x}_{2}}^{\mathrm{t}}$ induced by the inclusion

$$
\mathfrak{E}_{\tilde{x}_{1}} \rightarrow \mathfrak{D}_{\tilde{x}_{2}}
$$

is the $\mathfrak{e}_{\tilde{x}_{1}}$-th power map $\left[\mathfrak{e}_{\tilde{x}_{1}}\right]: \mathfrak{I}_{\tilde{x}_{2}}^{\mathrm{t}} \rightarrow \mathfrak{I}_{\tilde{x}_{2}}^{\mathrm{t}}$, as is easily verified. We define

$$
\tau_{\tilde{x}_{1}, \tilde{x}_{2}}^{\mathrm{t}}: \mathfrak{I}_{\tilde{x}_{1}}^{\mathrm{t}} \stackrel{\sim}{\rightarrow} \mathfrak{I}_{\tilde{x}_{2}}^{\mathrm{t}}
$$

to be the natural isomorphism obtained by composing the natural isomorphism

$$
\mathfrak{I}_{x_{1}}^{\mathrm{t}} \stackrel{\sim}{\rightarrow} \mathfrak{J}_{\tilde{x}_{1}}^{\mathrm{t}}
$$

induced by $\sigma$ (see Proposition 2.1(v)) with the natural identification $\mathfrak{J}_{\tilde{x}_{1}}^{\mathrm{t}} \stackrel{\sim}{\rightarrow} \mathfrak{I}_{\tilde{x}_{2}}^{\mathrm{t}}$.

The inertia-rigidity is equivalent to requiring the commutativity of the diagram

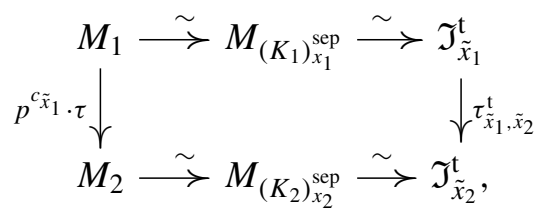

in which both vertical arrows are isomorphisms.

Define $\operatorname{Hom}\left(K_{2}, K_{1}\right)^{\text {sep }} \subset \operatorname{Hom}\left(K_{2}, K_{1}\right)$ to be the set of separable homomorphisms $K_{2} \rightarrow K_{1}$. Define $\operatorname{Hom}\left(\mathfrak{G}_{1}, \mathfrak{G}_{2}\right)^{\text {pr,inrig }} \subset \operatorname{Hom}\left(\mathfrak{G}_{1}, \mathfrak{G}_{2}\right)$ to be the set of proper (and hence continuous and open) inertia-rigid homomorphisms $\mathfrak{G}_{1} \rightarrow \mathfrak{G}_{2}$. Our aim in this section is to prove the following.

Theorem 4.8. The natural map $\operatorname{Hom}\left(K_{2}, K_{1}\right) \rightarrow \operatorname{Hom}\left(\mathfrak{G}_{1}, \mathfrak{G}_{2}\right) / \operatorname{Inn}\left(\mathfrak{G}_{2}\right)$ induces a bijection

$$
\operatorname{Hom}\left(K_{2}, K_{1}\right)^{\text {sep }} \stackrel{\sim}{\rightarrow} \operatorname{Hom}\left(\mathfrak{G}_{1}, \mathfrak{G}_{2}\right)^{\mathrm{pr}, \text { inrig }} / \operatorname{Inn}\left(\mathfrak{G}_{2}\right)
$$


More precisely:

(i) If $\gamma: K_{2} \rightarrow K_{1}$ is a separable homomorphism between fields, then the homomorphism $\mathfrak{G}_{1} \rightarrow \mathfrak{G}_{2}$ induced by $\gamma$ (up to inner automorphisms) is proper and inertia-rigid.

(ii) If $\sigma: \mathfrak{G}_{1} \rightarrow \mathfrak{G}_{2}$ is a proper, inertia-rigid homomorphism between profinite groups, then there exists a unique homomorphism $\tilde{\gamma}: \tilde{K}_{2} \rightarrow \tilde{K}_{1}$ of fields, such that $\tilde{\gamma} \circ \sigma\left(g_{1}\right)=g_{1} \circ \tilde{\gamma}$, for all $g_{1} \in \mathfrak{G}_{1}$, which induces a separable homomorphism $K_{2} \rightarrow K_{1}$.

Remark 4.9. (i) Assume that $\sigma: \mathfrak{G}_{1} \rightarrow \mathfrak{G}_{2}$ is a rigid homomorphism. Then it follows from Lemma 3.8(ii) that $\sigma$ is proper. Further, $\sigma$ is inertia-rigid. This can be reduced to the case where $\sigma$ is strictly rigid, and then deduced from class field theory as in the arguments preceding Lemma 4.12. (Note that then $\phi$ is bijective by Lemma 3.8(ii).) Thus, Theorem 4.8 can be viewed as a generalization of Theorem 3.4.

(ii) The natural map $\operatorname{Hom}\left(K_{2}^{\text {perf }}, K_{1}^{\text {perf }}\right) \rightarrow \operatorname{Hom}\left(\mathfrak{G}_{1}, \mathfrak{G}_{2}\right) / \operatorname{Inn}\left(\mathfrak{G}_{2}\right)$ induces a bijection

$$
\operatorname{Hom}\left(K_{2}^{\text {perf }}, K_{1}^{\text {perf }}\right) / \text { Frob }^{\mathbb{Z}} \stackrel{\sim}{\rightarrow} \operatorname{Hom}\left(\mathfrak{G}_{1}, \mathfrak{G}_{2}\right)^{\text {pr,inrig }} / \operatorname{Inn}\left(\mathfrak{G}_{2}\right) .
$$

Indeed, this follows from Theorem 4.8, since the natural map $\operatorname{Hom}\left(K_{2}, K_{1}\right) \rightarrow$ $\operatorname{Hom}\left(K_{2}^{\text {perf }}, K_{1}^{\text {perf }}\right)$ induces

$$
\operatorname{Hom}\left(K_{2}, K_{1}\right)^{\text {sep }} \stackrel{\sim}{\rightarrow} \operatorname{Hom}\left(K_{2}^{\text {perf }}, K_{1}^{\text {perf }}\right) / \text { Frob }^{\mathbb{Z}} .
$$

The rest of this section is devoted to the proof of Theorem 4.8.

First, to prove (i), let $\gamma: K_{2} \rightarrow K_{1}$ be a separable homomorphism. Then $\gamma$ induces naturally an open injective homomorphism $G_{1} \hookrightarrow G_{2}$ (up to $\operatorname{Inn}\left(G_{2}\right)$ ) and then an open homomorphism $\sigma: \mathfrak{G}_{1} \rightarrow \mathfrak{G}_{2}$ (up to $\operatorname{Inn}\left(\mathfrak{G}_{2}\right)$ ). The map $\sigma$ is well-behaved with respect to the map $\phi: \Sigma_{X_{1}} \rightarrow \Sigma_{X_{2}}$ that arises from a finite morphism $X_{1} \rightarrow X_{2}$ of schemes corresponding to $\gamma: K_{2} \rightarrow K_{1}$. Thus, each fiber of $\phi$ is finite, and hence $\sigma$ is proper. Next, if we define $\tau: M_{1} \stackrel{\sim}{\rightarrow} M_{2}$ to be the identification $M_{K_{1}^{\text {sep }}} \stackrel{\sim}{\rightarrow} M_{K_{2}^{\text {sep }}}$ (with respect to a suitable extension $K_{2}^{\text {sep }} \stackrel{\sim}{\rightarrow} K_{1}^{\text {sep }}$ of $\gamma: K_{2} \rightarrow K_{1}$ ), then diagram (4.1) commutes with $e_{\tilde{x}_{1}}$ defined to be the ramification index of $K_{1} / K_{2}$ at $\tilde{x}_{1}$. Thus, $\sigma$ is inertia-rigid.

Next, to prove (ii), let $\sigma: \mathfrak{G}_{1} \rightarrow \mathfrak{G}_{2}$ be a proper, inertia-rigid homomorphism.

Lemma 4.10. Condition 2 holds for $\sigma: \mathfrak{G}_{1} \rightarrow \mathfrak{G}_{2}$.

Proof. Same as that of Lemma 3.6.

Thus, we may apply Lemmas 2.6-2.9 to $\sigma$. 
Next, let $\tau: M_{1} \stackrel{\sim}{\rightarrow} M_{2}$ be the isomorphism appearing in the definition of inertiarigid homomorphism, so that diagram (4.1) commutes for each $\tilde{x}_{1} \in \Sigma_{\tilde{X}_{1}}$ and for some $e_{\tilde{x}_{1}} \in \mathbb{Z}_{>0}$.

Lemma 4.11. (i) The isomorphism $\tau: M_{1} \stackrel{\sim}{\rightarrow} M_{2}$ is Galois-equivariant with respect to $\sigma$.

(ii) The positive integers $e_{\tilde{x}_{1}}, \mathfrak{e}_{\tilde{x}_{1}}$ and $\mathfrak{e}_{\tilde{x}_{1}}^{\mathrm{t}}$ depend only on the image $x_{1} \in \Sigma_{X_{1}}$ of $\tilde{x}_{1}$.

Proof. (i) For each $\tilde{x}_{1} \in \Sigma_{\tilde{X}_{1}}$, the commutativity of diagram (4.1), together with Proposition 2.1(iv), implies that $\tau$ is Galois-equivariant with respect to

$$
\mathfrak{D}_{\tilde{x}_{1}} \stackrel{\sigma}{\rightarrow} \mathfrak{D}_{\tilde{\phi}\left(\tilde{x}_{1}\right)}
$$

Our assertion then follows, since $\mathfrak{G}_{1}$ is generated by the decomposition subgroups $\mathfrak{D}_{\tilde{x}_{1}}$ for all $\tilde{x}_{1} \in \Sigma_{\tilde{X}_{1}}$, as follows from Chebotarev's density theorem.

(ii) Take another $\tilde{x}_{1}^{\prime} \in \Sigma_{\tilde{X}_{1}}$ above $x_{1} \in \Sigma_{X_{1}}$ and set $\tilde{x}_{2}^{\prime} \stackrel{\text { def }}{=} \tilde{\phi}\left(\tilde{x}_{1}^{\prime}\right)$. Fix $\gamma \in \mathfrak{G}_{1}$ such that $\tilde{x}_{1}^{\prime}=\gamma \tilde{x}_{1}$. By the Galois-equivariance property of $\tilde{\phi}$ (Remark 4.2(iv)), we have then $\tilde{x}_{2}^{\prime}=\sigma(\gamma) \tilde{x}_{2}$. Denote by $[\gamma]$ and $[\sigma(\gamma)]$ the inner automorphisms of $\mathfrak{G}_{1}$ and $\mathfrak{G}_{2}$ induced by $\gamma$ and $\sigma(\gamma)$, respectively. We have the commutative diagram

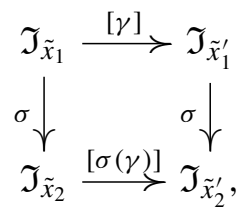

in which both rows are isomorphisms. It follows that $\mathfrak{e}_{\tilde{x}_{1}^{\prime}}=\mathfrak{e}_{\tilde{x}_{1}}$. This commutative diagram induces the commutative diagram

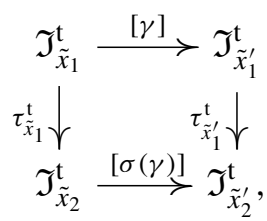

in which both rows are isomorphisms. It follows that $\mathfrak{e}_{\tilde{x}_{1}^{\prime}}^{\mathrm{t}}=\mathfrak{e}_{\tilde{x}_{1}}^{\mathrm{t}}$. With (i), this last commutative diagram also implies that $e_{\tilde{x}_{1}^{\prime}}=e_{\tilde{x}_{1}}$.

From now on, we shall write $e_{x_{1}}, \mathfrak{e}_{x_{1}}$ and $\mathfrak{e}_{x_{1}}^{\mathrm{t}}$ for $e_{\tilde{x}_{1}}, \mathfrak{e}_{\tilde{x}_{1}}$ and $\mathfrak{e}_{\tilde{x}_{1}}^{\mathrm{t}}$, respectively. Further, according to this, we shall write $a_{x_{1}}, b_{x_{1}}$ and $c_{x_{1}}$ for the invariants $a_{\tilde{x}_{1}}, b_{\tilde{x}_{1}}$ and $c_{\tilde{x}_{1}}$ in Remark 4.7(i), respectively. We may and shall also assume that

$$
a\left(=\min \left\{a_{x_{1}} \mid x_{1} \in \Sigma_{X_{1}}\right\}\right)=0
$$

see Remark 4.7(i). 
We have the commutative diagram of exact sequences

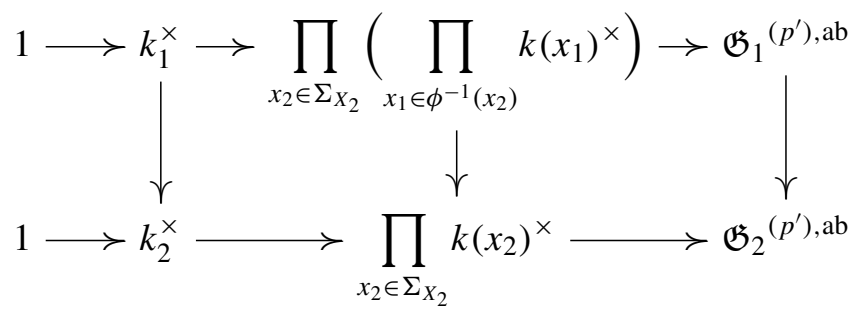

from global class field theory. Here, the map $\mathfrak{G}_{1}{ }^{\left(p^{\prime}\right), \mathrm{ab}} \rightarrow \mathfrak{G}_{2}{ }^{\left(p^{\prime}\right) \text {,ab }}$ is naturally induced by $\sigma$. The right horizontal maps are induced by Artin's reciprocity map, and the map

$$
\prod_{x_{2} \in \Sigma_{X_{2}}}\left(\prod_{x_{1} \in \phi^{-1}\left(x_{2}\right)} k\left(x_{1}\right)^{\times}\right) \rightarrow \prod_{x_{2} \in \Sigma_{X_{2}}} k\left(x_{2}\right)^{\times}
$$

maps each component $k\left(x_{1}\right)^{\times}$to $k\left(x_{2}\right)^{\times}$as follows. First, $k\left(x_{1}\right)^{\times}$maps isomorphically onto $\ell_{x_{1}}^{\times}$via the natural identification induced by $\sigma$; see Remark 4.7(ii) and Proposition 2.1(iii). Then $\ell_{x_{1}}^{\times}$maps to $k\left(x_{2}\right)^{\times}$by the $\mathfrak{e}_{x_{1}}$-th power of the norm map.

The above diagram induces, for each $x_{2} \in \Sigma_{X_{2}}$, the commutative diagram

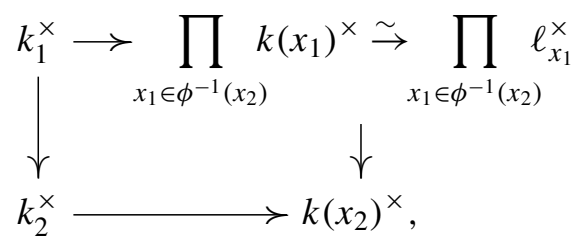

where the map $k_{2}^{\times} \rightarrow k\left(x_{2}\right)^{\times}$is the natural embedding, the map

$$
k_{1}^{\times} \rightarrow \prod_{x_{1} \in \phi^{-1}\left(x_{2}\right)} k\left(x_{1}\right)^{\times}
$$

is the natural diagonal embedding, and the isomorphism

$$
\prod_{x_{1} \in \phi^{-1}\left(x_{2}\right)} k\left(x_{1}\right)^{\times} \stackrel{\sim}{\rightarrow} \prod_{x_{1} \in \phi^{-1}\left(x_{2}\right)} \ell_{x_{1}}^{\times}
$$

and the map

$$
\prod_{x_{1} \in \phi^{-1}\left(x_{2}\right)} \ell_{x_{1}}^{\times} \rightarrow k\left(x_{2}\right)^{\times}
$$

are as above. By passing to various open subgroups corresponding to extensions of the constant fields, and to the projective limit via the norm maps, we obtain the 
commutative diagram

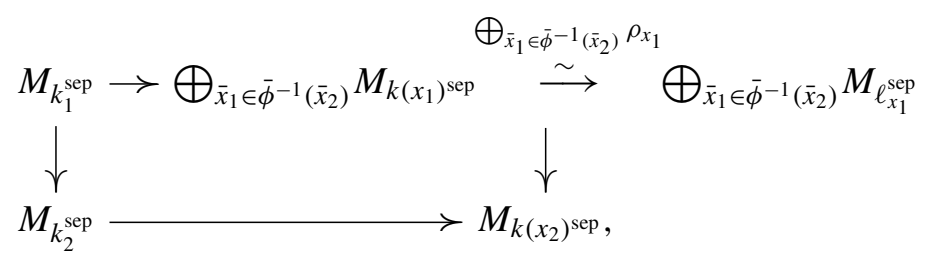

where

$$
\rho_{x_{1}}: M_{k\left(x_{1}\right)^{\text {sep }}} \stackrel{\sim}{\rightarrow} M_{\ell_{x_{1}}^{\text {sep }}}
$$

is the natural isomorphism induced by $\sigma$; see Remark 4.7(ii) and Proposition 2.1(v). Here, $\bar{x}_{2} \in \Sigma_{\bar{X}_{2}}$ is any point above $x_{2}$ and $\bar{\phi}: \Sigma_{\bar{X}_{1}} \rightarrow \Sigma_{\bar{X}_{2}}$ is obtained as the inductive limit of $\phi$ 's for various open subgroups corresponding to extensions of the constant fields. Observe that $\bar{\phi}: \Sigma_{\bar{X}_{1}} \rightarrow \Sigma_{\bar{X}_{2}}$ has finite fibers, since $\phi: \Sigma_{X_{1}} \rightarrow \Sigma_{X_{2}}$ has finite fibers, the projection $\Sigma_{\bar{X}_{1}} \rightarrow \Sigma_{X_{1}}$ has finite fibers, and $\bar{\phi}$ is compatible with $\phi$.

This can be rewritten as

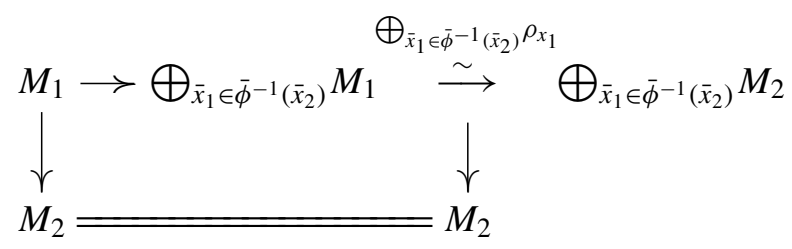

via the natural identifications $M_{k\left(x_{1}\right)^{\text {sep }}} \stackrel{\sim}{\rightarrow} M_{1}$ and $M_{\ell_{1}}$ sep $\stackrel{\sim}{\rightarrow} M_{2}$ for $x_{1} \in \phi^{-1}\left(x_{2}\right)$; $M_{k\left(x_{2}\right)^{\text {sep }}} \stackrel{\sim}{\rightarrow} M_{2}$; and $M_{k_{i}^{\text {sep }}} \stackrel{\sim}{\rightarrow} M_{i}, i=1,2$. Thus, in diagram (4.2) the map $M_{1} \rightarrow$ $\bigoplus_{\bar{x}_{1} \in \bar{\phi}^{-1}\left(\bar{x}_{2}\right)} M_{1}$ is the natural diagonal embedding, and the map $\bigoplus_{\bar{x}_{1} \in \bar{\phi}^{-1}\left(\bar{x}_{2}\right)} M_{2} \rightarrow$ $M_{2}$ is the map $\oplus_{\bar{x}_{1} \in \bar{\phi}^{-1}\left(\bar{x}_{2}\right)}\left[\mathfrak{e}_{x_{1}}\right]$. We shall denote by $\tau^{\prime}: M_{1} \rightarrow M_{2}$ the homomorphism that is the left vertical arrow in diagram (4.2) (note that $\tau^{\prime}$ is independent of the choice of $x_{2} \in \Sigma_{X_{2}}$ ).

Lemma 4.12 (product formula). The sum $\sum_{\bar{x}_{1} \in \bar{\phi}^{-1}\left(\bar{x}_{2}\right)} e_{x_{1}}$ is independent of the choice of $x_{2} \in \Sigma_{X_{2}}$. Set $n \stackrel{\text { def }}{=} \sum_{\bar{x}_{1} \in \bar{\phi}^{-1}\left(\bar{x}_{2}\right)} e_{x_{1}}>0$. Then we have $\tau^{\prime}=[n] \circ \tau$, where $[n]: M_{2} \rightarrow M_{2}$ denotes the map of elevation to the power $n$ in $M_{2}$.

Proof. This follows from the commutativity of diagram (4.2), by observing that the homomorphism $\sigma$ being inertia-rigid means that the isomorphism $\rho_{x_{1}}$ in diagram (4.2) equals $p^{c_{x_{1}}} \tau$ for all $\bar{x}_{1} \in \bar{\phi}^{-1}\left(\bar{x}_{2}\right)$.

For the rest of this section, all cohomology groups will be continuous Galois cohomology groups unless otherwise specified.

The Galois-equivariant identification $\tau^{-1}: M_{2} \stackrel{\sim}{\rightarrow} M_{1}$ induces naturally an injective homomorphism $H^{1}\left(\mathfrak{G}_{2}, M_{2}\right) \rightarrow H^{1}\left(\mathfrak{G}_{1}, M_{1}\right)$ between Galois cohomology 
groups. Indeed, this homomorphism fits into the commutative diagram

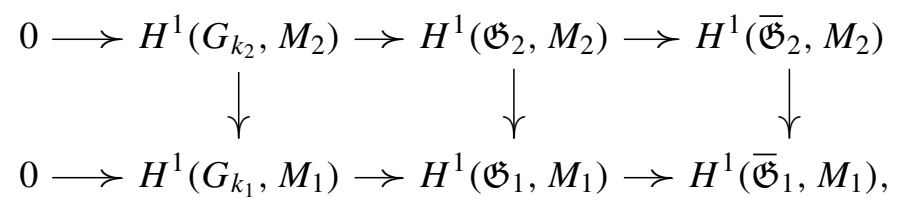

where both rows are exact and vertical maps are natural maps induced by $\left(\sigma, \tau^{-1}\right)$. Here, the left vertical arrow is injective by $H^{0}\left(H_{k_{1}}, M_{2}\right)=0$, where $H_{k_{1}}$ stands for the (isomorphic) image of $G_{k_{1}}$ in $G_{k_{2}}$, and the right vertical arrow is injective since $M_{2}$ is torsion-free and $\left[\overline{\mathfrak{G}}_{2}: \sigma\left(\overline{\mathfrak{G}}_{1}\right)\right]<\infty$. Therefore, the middle vertical arrow is also injective.

Further, for each $x_{2} \in \Sigma_{X_{2}}$, the following diagram is commutative:

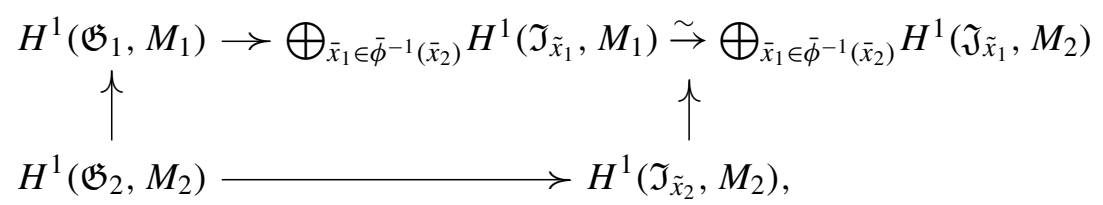

where the horizontal maps are the natural restriction maps, the left vertical map is the above map, the map

$$
H^{1}\left(\mathfrak{I}_{\tilde{x}_{2}}, M_{2}\right) \rightarrow \bigoplus_{\bar{x}_{1} \in \bar{\phi}^{-1}\left(\bar{x}_{2}\right)} H^{1}\left(\mathfrak{J}_{\tilde{x}_{1}}, M_{2}\right)
$$

is the natural map induced by the inclusion $\mathfrak{J}_{\tilde{x}_{1}} \subset \mathfrak{I}_{\tilde{x}_{2}}$ for $\bar{x}_{1} \in \bar{\phi}^{-1}\left(\bar{x}_{2}\right)$, and the isomorphism $H^{1}\left(\mathfrak{I}_{\tilde{x}_{1}}, M_{1}\right) \stackrel{\sim}{\rightarrow} H^{1}\left(\mathfrak{J}_{\tilde{x}_{1}}, M_{2}\right)$ is naturally induced by the natural surjective map $\mathfrak{I}_{\tilde{x}_{1}} \rightarrow \mathfrak{J}_{\tilde{x}_{1}}$, which is induced by $\left(\sigma, \tau^{-1}\right)$.

We have natural identifications

$$
\begin{aligned}
& H^{1}\left(\mathfrak{I}_{\tilde{x}_{1}}, M_{1}\right) \stackrel{\sim}{\rightarrow} \operatorname{Hom}\left(\mathfrak{I}_{\tilde{x}_{1}}, M_{1}\right) \stackrel{\sim}{\rightarrow} \operatorname{Hom}\left(\mathfrak{I}_{\tilde{x}_{1}}^{\mathrm{t}}, M_{1}\right) \stackrel{\sim}{\rightarrow} \operatorname{Hom}\left(M_{1}, M_{1}\right) \stackrel{\sim}{\rightarrow} \hat{\mathbb{Z}}^{p^{\prime}}, \\
& H^{1}\left(\mathfrak{J}_{\tilde{x}_{1}}, M_{2}\right) \stackrel{\sim}{\rightarrow} \operatorname{Hom}\left(\mathfrak{J}_{\tilde{x}_{1}}, M_{2}\right) \stackrel{\sim}{\rightarrow} \operatorname{Hom}\left(\mathfrak{J}_{\tilde{x}_{1}}^{\mathrm{t}}, M_{2}\right) \stackrel{\sim}{\rightarrow} \operatorname{Hom}\left(M_{2}, M_{2}\right) \stackrel{\sim}{\rightarrow} \hat{\mathbb{Z}}^{p^{\prime}}, \\
& H^{1}\left(\mathfrak{I}_{\tilde{x}_{2}}, M_{2}\right) \stackrel{\sim}{\rightarrow} \operatorname{Hom}\left(\mathfrak{I}_{\tilde{x}_{2}}, M_{2}\right) \stackrel{\sim}{\rightarrow} \operatorname{Hom}\left(\mathfrak{I}_{\tilde{x}_{2}}^{\mathrm{t}}, M_{2}\right) \stackrel{\sim}{\rightarrow} \operatorname{Hom}\left(M_{2}, M_{2}\right) \stackrel{\sim}{\rightarrow} \hat{\mathbb{Z}}^{p^{\prime}} .
\end{aligned}
$$

In light of these identifications, the above diagram can be rewritten as

$$
\begin{gathered}
H^{1}\left(\mathfrak{G}_{1}, M_{1}\right) \longrightarrow \bigoplus_{\bar{x}_{1} \in \bar{\phi}^{-1}\left(\bar{x}_{2}\right)} \hat{\mathbb{Z}}^{p^{\prime}} \\
\uparrow \quad \begin{array}{l}
\oplus_{\bar{x}_{1} \in \bar{\phi}^{-1}\left(\bar{x}_{2}\right)}\left[e_{x_{1}}\right] \uparrow \\
H^{1}\left(\mathfrak{G}_{2}, M_{2}\right)
\end{array} \\
\longrightarrow
\end{gathered}
$$

where the vertical map $\hat{\mathbb{Z}}^{p^{\prime}} \rightarrow \bigoplus_{\bar{x}_{1} \in \bar{\phi}^{-1}\left(\bar{x}_{2}\right)} \hat{\mathbb{Z}}^{p^{\prime}}$ is the map $\bigoplus_{\bar{x}_{1} \in \phi^{-1}\left(\bar{x}_{2}\right)}\left[e_{x_{1}}\right]$, and $\left[e_{x_{1}}\right]$ denotes the map of multiplication by $e_{x_{1}}$ in $\hat{\mathbb{Z}}^{p^{\prime}}$. By considering all $x_{2} \in \Sigma_{X_{2}}$, 
we obtain the following commutative diagram:

$$
\begin{gathered}
H_{\uparrow}^{1}\left(\mathfrak{G}_{1}, M_{1}\right) \rightarrow \widehat{\operatorname{Div}}_{\bar{X}_{1}} \stackrel{\text { def }}{=} \prod_{\bar{x}_{1} \in \Sigma_{\bar{X}_{1}}}^{\prime} \hat{\mathbb{Z}}^{p^{\prime}} \stackrel{\sim}{\rightarrow} \prod_{\bar{x}_{2} \in \Sigma_{\bar{X}_{2}}}^{\prime}\left(\bigoplus_{\bar{x}_{1} \in \phi^{-1}\left(\bar{x}_{2}\right)} \hat{\mathbb{Z}}^{p^{\prime}}\right) \\
H^{1}\left(\mathfrak{G}_{2}, M_{2}\right) \longrightarrow \widehat{\operatorname{Div}}_{\bar{X}_{2}} \stackrel{\text { def }}{=} \prod_{\bar{x}_{2} \in \Sigma_{\bar{X}_{2}}}^{\prime} \hat{\mathbb{Z}}^{p^{\prime}} .
\end{gathered}
$$
Here, given an index set $\Lambda$, we define $\prod_{\lambda \in \Lambda}^{\prime} \hat{\mathbb{Z}}^{p^{\prime}} \stackrel{\text { def }}{=} \underset{p \nmid n}{\lim }\left(\bigoplus_{\lambda \in \Lambda} \mathbb{Z} / n \mathbb{Z}\right.$ ). (Accord-
ingly, one has

$$
\bigoplus_{\lambda \in \Lambda} \hat{\mathbb{Z}}^{p^{\prime}} \subset \prod_{\lambda \in \Lambda}^{\prime} \hat{\mathbb{Z}}^{p^{\prime}} \subset \prod_{\lambda \in \Lambda} \hat{\mathbb{Z}}^{p^{\prime}}
$$

and the equalities hold if and only if $\sharp(\Lambda)<\infty$.) Thus, the map $\widehat{\operatorname{Div}}_{\bar{X}_{2}} \rightarrow \widehat{\operatorname{Div}}_{\bar{X}_{1}}$ maps $\bar{x}_{2}$ to $\sum_{\bar{x}_{1} \in \phi^{-1}\left(\bar{x}_{2}\right)} e_{x_{1}} \bar{x}_{1}$. In particular, the subgroup $\widehat{\operatorname{Div}}_{X_{2}}$ of $\widehat{\operatorname{Div}}_{\bar{X}_{2}}$ maps into the subgroup $\widehat{\operatorname{Div}}_{X_{1}}$ of $\widehat{\operatorname{Div}}_{\bar{X}_{1}}$. Here, for $i=1,2$,

$$
\widehat{\operatorname{Div}}_{X_{i}} \stackrel{\text { def }}{=} \prod_{x_{1} \in \Sigma_{X_{i}}}^{\prime} \hat{\mathbb{Z}}^{p^{\prime}}
$$

is naturally embedded into $\widehat{\operatorname{Div}}_{\bar{X}_{i}}$ and is regarded as a subgroup of $\widehat{\operatorname{Div}}_{\bar{X}_{i}}$. It follows from various constructions that, for $i=1,2$, the image of the map $H^{1}\left(\mathfrak{G}_{i}, M_{i}\right) \rightarrow$ $\widehat{\operatorname{Div}}_{\bar{X}_{i}}$ is contained in $\widehat{\operatorname{Div}}_{X_{i}}$. Thus, we obtain the commutative diagram

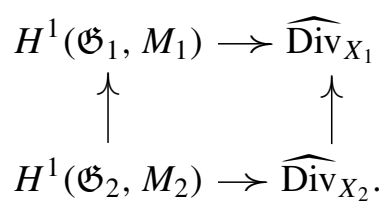

For $i=1,2$, set $\operatorname{Div}_{X_{i}} \stackrel{\text { def }}{=} \bigoplus_{x_{i} \in \Sigma_{X_{i}}} \mathbb{Z}$, which is the group of divisors on $X_{i}$. Then the subgroup $\operatorname{Div}_{X_{2}}$ of $\widehat{\operatorname{Div}}_{X_{2}}$ maps into the subgroup

$$
\operatorname{Div}_{X_{1}}=\bigoplus_{x_{2} \in \Sigma_{X_{2}}}\left(\bigoplus_{x_{1} \in \phi^{-1}\left(x_{2}\right)} \mathbb{Z}\right)
$$

of $\widehat{\operatorname{Div}}_{X_{1}}$. Thus, we have a natural map

$$
\operatorname{Div}_{X_{2}} \rightarrow \operatorname{Div}_{X_{1}}
$$

We denote by $\operatorname{Pri}_{X_{i}}$ the subgroup of $\operatorname{Div}_{X_{i}}$ which consists of principal divisors. Note that we have a natural map $K_{i}^{\times} \rightarrow \operatorname{Div}_{X_{i}}$, which maps a function $f_{i}$ to its $\operatorname{divisor} \operatorname{div}\left(f_{i}\right)$ of zeros and poles. Further, Let $J_{X_{i}}$ be the Jacobian variety of $X_{i}$. 
Let $\operatorname{Div}_{X_{i}}^{0} \subset \operatorname{Div}_{X_{i}}$ be the group of degree-zero divisors on $X_{i}$. Then there exists a natural isomorphism

$$
\operatorname{Div}_{X_{i}}^{0} / \operatorname{Pri}_{X_{i}}=J_{X_{i}}\left(k_{i}\right) .
$$

Write $D_{X_{i}}$ for the kernel of the natural homomorphism $\operatorname{Div}_{X_{i}}^{0} \rightarrow J_{X_{i}}\left(k_{i}\right)^{p^{\prime}}$, with $J_{X_{i}}\left(k_{i}\right)^{p^{\prime}}$ standing for the maximal prime-to- $p$ quotient $J_{X_{i}}\left(k_{i}\right) /\left(J_{X_{i}}\left(k_{i}\right)\{p\}\right)$ of $J_{X_{i}}\left(k_{i}\right)$, where, for an abelian group $M, M\{p\}$ stands for the subgroup of torsion elements $a$ of $M$ of $p$-power order. Then $D_{X_{i}}$ sits naturally in the exact sequence

$$
0 \rightarrow \operatorname{Pri}_{X_{i}} \rightarrow D_{X_{i}} \rightarrow J_{X_{i}}\left(k_{i}\right)\{p\} \rightarrow 0 .
$$

For $i \in\{1,2\}$, and a positive integer $n$ prime to $p$, the Kummer exact sequence

$$
1 \rightarrow \mu_{n} \rightarrow \mathbb{G}_{m} \stackrel{[n]}{\longrightarrow} \mathbb{G}_{m} \rightarrow 1
$$

induces a natural isomorphism

$$
K_{i}^{\times} /\left(K_{i}^{\times}\right)^{n} \stackrel{\sim}{\rightarrow} H^{1}\left(\mathfrak{G}_{i}, \mu_{n}\left(K_{i}^{\mathrm{sep}}\right)\right) ;
$$

see Lemma 1.4. By passing to the projective limit over all integers $n$ prime to $p$, we obtain a natural isomorphism

$$
\left(K_{i}^{\times}\right)^{\wedge p^{\prime}} \stackrel{\sim}{\rightarrow} H^{1}\left(\mathfrak{G}_{i}, M_{i}\right)
$$

where

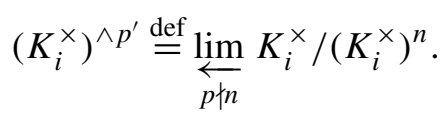

Since we have a natural embedding $K_{i}^{\times} \hookrightarrow\left(K_{i}^{\times}\right)^{\wedge p^{\prime}}$, we get a natural embedding

$$
K_{i}^{\times} \hookrightarrow H^{1}\left(\mathfrak{G}_{i}, M_{i}\right)
$$

In what follows we will identify $K_{i}^{\times}$with its image in $H^{1}\left(\mathfrak{G}_{i}, M_{i}\right) ; i=1,2$. The natural maps $K_{i}^{\times} \rightarrow \operatorname{Div}_{X_{i}}$ and $H^{1}\left(\mathfrak{G}_{i}, M_{i}\right) \rightarrow \widehat{\operatorname{Div}}_{X_{i}}$ are compatible with each other, and hence the image of $K_{i}^{\times}$in $\widehat{\operatorname{Div}}_{X_{i}}$, via the map $H^{1}\left(\mathfrak{G}_{i}, M_{i}\right) \rightarrow \widehat{\operatorname{Div}}_{X_{i}}$ in diagram (4.3), coincides with the subgroup $\operatorname{Pri}_{X_{i}}$ of principal divisors.

Lemma 4.13 (recovering the multiplicative group). (i) The homomorphism

$$
\widehat{\operatorname{Div}}_{X_{2}} \rightarrow \widehat{\operatorname{Div}}_{X_{1}}
$$

in diagram (4.3) maps $D_{X_{2}}$ into $D_{X_{1}}$.

(ii) The above map $H^{1}\left(\mathfrak{G}_{2}, M_{2}\right) \rightarrow H^{1}\left(\mathfrak{G}_{1}, M_{1}\right)$ induces a natural injective (multiplicative) homomorphism

$$
\gamma: K_{2}^{\times} \hookrightarrow\left(K_{1}^{\times}\right)^{p^{-n}}=\left(K_{1}^{p^{-n}}\right)^{\times},
$$

where $p^{n}$ is the exponent of the p-primary finite abelian group $J_{X_{1}}\left(k_{1}\right)\{p\}$. We have $\left[\gamma\left(K_{2}^{\times}\right): \gamma\left(K_{2}^{\times}\right) \cap K_{1}^{\times}\right]<\infty$ and $\left[\gamma\left(K_{2}^{\times}\right): \gamma\left(K_{2}^{\times}\right) \cap\left(K_{1}^{\times}\right)^{p}\right]>1$. 
Moreover, this injective homomorphism is functorial in the following sense: Let $\mathfrak{H}_{1} \subset \mathfrak{G}_{1}, \mathfrak{H}_{2} \subset \mathfrak{G}_{2}$ be open subgroups such that $\sigma\left(\mathfrak{H}_{1}\right) \subset \mathfrak{H}_{2}$, and, for $i=1$, 2, let $L_{i} / K_{i}$ be the finite separable extension corresponding to $\mathfrak{H}_{i} \subset \mathfrak{G}_{i}, Y_{i}$ the integral closure of $X_{i}$ in $L_{i}$, and $\ell_{i}$ the constant field of $L_{i}$ (that is, the algebraic closure of $k_{i}$ in $\left.L_{i}\right)$. Then we have a commutative diagram

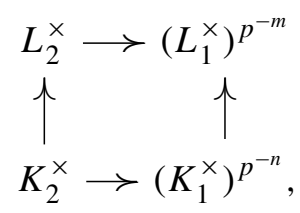

where $p^{m} \geq p^{n}$ is the exponent of the p-primary finite abelian group $J_{Y_{1}}\left(\ell_{1}\right)\{p\}$, and the vertical arrows are the natural embeddings.

Proof. (i) We have the diagram of maps

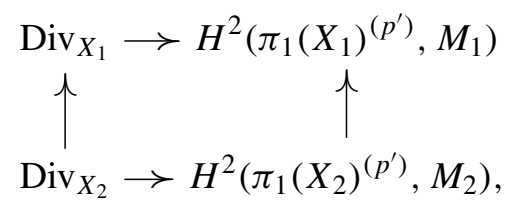

where the map $\operatorname{Div}_{X_{2}} \rightarrow \operatorname{Div}_{X_{1}}$ is the one induced by the map $\widehat{\operatorname{Div}}_{X_{2}} \rightarrow \widehat{\operatorname{Div}}_{X_{1}}$ in diagram (4.3). For $i \in\{1,2\}$, the group $H^{2}\left(\pi_{1}\left(X_{i}\right)^{\left(p^{\prime}\right)}, M_{i}\right)$ denotes the second cohomology group of the profinite group $\pi_{1}\left(X_{i}\right)^{\left(p^{\prime}\right)}$ with coefficients in the (continuous) $\pi_{1}\left(X_{i}\right)^{\left(p^{\prime}\right)}$-module $M_{i}$.

First, we shall treat the special case that $\left(g_{1} \geq\right) g_{2}>0$. In this case, we have a natural isomorphism $H^{2}\left(\pi_{1}\left(X_{i}\right)^{\left(p^{\prime}\right)}, M_{i}\right) \stackrel{\sim}{\rightarrow} H_{\text {et }}^{2}\left(X_{i}, M_{i}\right)$ ([Mochizuki 2007, Proposition 1.1]), where $H_{\mathrm{et}}^{2}\left(X_{i}, M_{i}\right)$ denotes the second étale cohomology group of $X_{i}$ with coefficients in $M_{i}$. We will identify the groups $H^{2}\left(\pi_{1}\left(X_{i}\right)^{\left(p^{\prime}\right)}, M_{i}\right)$ and $H_{\mathrm{et}}^{2}\left(X_{i}, M_{i}\right)$ via the above identifications. Further, the map $H^{2}\left(\pi_{1}\left(X_{2}\right)^{\left(p^{\prime}\right)}, M_{2}\right) \rightarrow$ $H^{2}\left(\pi_{1}\left(X_{1}\right)^{\left(p^{\prime}\right)}, M_{1}\right)$ is the map induced by the natural map $\pi_{1}\left(X_{1}\right)^{\left(p^{\prime}\right)} \rightarrow \pi_{1}\left(X_{2}\right)^{\left(p^{\prime}\right)}$ between fundamental groups, which is induced by $\sigma$ (see Lemma 2.6), and the Galois-equivariant identification $\tau^{-1}: M_{2} \stackrel{\sim}{\rightarrow} M_{1}$. The map

$$
\operatorname{Div}_{X_{i}} \rightarrow H^{2}\left(\pi_{1}\left(X_{i}\right)^{\left(p^{\prime}\right)}, M_{i}\right)
$$

maps a divisor $D$ to its first arithmetic (étale) Chern class $c_{1}(D)$, and is naturally induced by the Kummer exact sequence

$$
1 \rightarrow \mu_{n} \rightarrow \mathbb{G}_{m} \stackrel{[n]}{\longrightarrow} \mathbb{G}_{m} \rightarrow 1
$$

in étale topology (see [Mochizuki 2003, 4.1]). In particular, the map $\operatorname{Div}_{X_{i}} \rightarrow$ $H^{2}\left(\pi_{1}\left(X_{i}\right)^{\left(p^{\prime}\right)}, M_{i}\right)$ factors as

$$
\operatorname{Div}_{X_{i}} \rightarrow \operatorname{Pic}\left(X_{i}\right) /\left(J_{X_{i}}\left(k_{i}\right)\{p\}\right) \hookrightarrow H^{2}\left(\pi_{1}\left(X_{i}\right)^{\left(p^{\prime}\right)}, M_{i}\right),
$$


where $\operatorname{Pic}\left(X_{i}\right) \stackrel{\text { def }}{=} H_{\mathrm{et}}^{1}\left(X_{i}, \mathbb{G}_{m}\right)$ is the Picard group of $X_{i}$. Note that the kernel of the above map $\operatorname{Div}_{X_{i}} \rightarrow H^{2}\left(\pi_{1}\left(X_{i}\right)^{\left(p^{\prime}\right)}, M_{i}\right)$ coincides with $D_{X_{i}}$. We claim that the above diagram is commutative. Thus, it induces a natural map $D_{X_{2}} \rightarrow D_{X_{1}}$, as desired (in the case that $g_{2}>0$ ).

To prove this claim, let $x_{2} \in \Sigma_{X_{2}}$. We shall investigate the images of $x_{2} \in$ $\operatorname{Div}_{X_{2}}$ in $H^{2}\left(\pi_{1}\left(X_{1}\right)^{\left(p^{\prime}\right)}, M_{1}\right)$ under the two (composite) maps in the above diagram. First, consider the special case where $x_{2} \in \Sigma_{X_{2}}$ is $k_{2}$-rational and each point of $\phi^{-1}\left(x_{2}\right) \subset \Sigma_{X_{1}}$ is $k_{1}$-rational. Then the image $c_{1}\left(x_{2}\right)$ of the divisor $x_{2} \in \operatorname{Div}_{X_{2}}$ in $H^{2}\left(\pi_{1}\left(X_{2}\right)^{\left(p^{\prime}\right)}, M_{2}\right)$ coincides with the class of the extension $1 \rightarrow$ $M_{2} \rightarrow \pi_{1}\left(\mathbb{L}_{x_{2}}^{\times}\right)^{\left(p^{\prime}\right)} \rightarrow \pi_{1}\left(X_{2}\right)^{\left(p^{\prime}\right)} \rightarrow 1$, where $\pi_{1}\left(\mathbb{L}_{x_{2}}^{\times}\right)^{\left(p^{\prime}\right)}$ is the geometrically primeto- $p$ fundamental group of the line bundle $\mathbb{L}_{x_{2}}$ corresponding to the invertible sheaf $\mathrm{O}_{X_{2}}\left(x_{2}\right)$ with the zero section removed [Mochizuki 2005, Lemma 4.2; Mochizuki 2003, 4.1]. Further, $\pi_{1}\left(\mathbb{L}_{x_{2}}^{\times}\right)^{\left(p^{\prime}\right)}$ is naturally identified with the maximal cuspidally central quotient $\pi_{1}\left(X_{2} \backslash\left\{x_{2}\right\}\right)^{\left(p^{\prime}\right) \text {, c-cn }}$ of $\pi_{1}\left(X_{2} \backslash\left\{x_{2}\right\}\right)^{\left(p^{\prime}\right)}$. Here, for a nonempty open subscheme $U_{i} \subset X_{i}$, we define the maximal (geometrically prime-to- $p$ ) cuspidally central quotient $\pi_{1}\left(U_{i}\right)^{\left(p^{\prime}\right), \mathrm{c}-\mathrm{cn}}$ to be the maximal quotient of $\pi_{1}\left(U_{i}\right)^{\left(p^{\prime}\right)}$ in which the image of $\operatorname{Ker}\left(\pi_{1}\left(\bar{U}_{i}\right) \rightarrow \pi_{1}\left(\bar{X}_{i}\right)\right)$ lies in the center of the image of $\pi_{1}\left(\bar{U}_{i}\right)$ [Mochizuki 2005, Lemma 4.2(iii)]. Similarly, the maximal cuspidally central quotient $\pi_{1}\left(X_{1} \backslash \phi^{-1}\left(x_{2}\right)\right)^{\left(p^{\prime}\right), \mathrm{c}-\mathrm{cn}}$ of $\pi_{1}\left(X_{1} \backslash \phi^{-1}\left(x_{2}\right)\right)^{\left(p^{\prime}\right)}$ gives the extension of $\pi_{1}\left(X_{1}\right)^{\left(p^{\prime}\right)}$ by $\bigoplus_{x_{1} \in \phi^{-1}\left(x_{2}\right)} M_{1}$ that corresponds to

$$
\left(c_{1}\left(x_{1}\right)\right)_{x_{1} \in \phi^{-1}\left(x_{2}\right)} \in \bigoplus_{x_{1} \in \phi^{-1}\left(x_{2}\right)} H^{2}\left(\pi_{1}\left(X_{1}\right)^{\left(p^{\prime}\right)}, M_{1}\right)=H^{2}\left(\pi_{1}\left(X_{1}\right)^{\left(p^{\prime}\right)}, \bigoplus_{x_{1} \in \phi^{-1}\left(x_{2}\right)} M_{1}\right) .
$$

Being well-behaved (with respect to $\tilde{\phi}), \sigma$ induces naturally a homomorphism $\pi_{1}\left(X_{1} \backslash \phi^{-1}\left(x_{2}\right)\right)^{\left(p^{\prime}\right)} \rightarrow \pi_{1}\left(X_{2} \backslash\left\{x_{2}\right\}\right)^{\left(p^{\prime}\right)}$, which is a lifting of $\pi_{1}\left(X_{1}\right)^{\left(p^{\prime}\right)} \rightarrow$ $\pi_{1}\left(X_{2}\right)^{\left(p^{\prime}\right)}$ and which further induces a homomorphism $\pi_{1}\left(X_{1} \backslash \phi^{-1}\left(x_{2}\right)\right)^{\left(p^{\prime}\right), \mathrm{c}-\mathrm{cn}} \rightarrow$ $\pi_{1}\left(X_{2} \backslash\left\{x_{2}\right\}\right)^{\left(p^{\prime}\right), \mathrm{c}-\mathrm{cn}}$. These homomorphisms fit into the commutative diagram

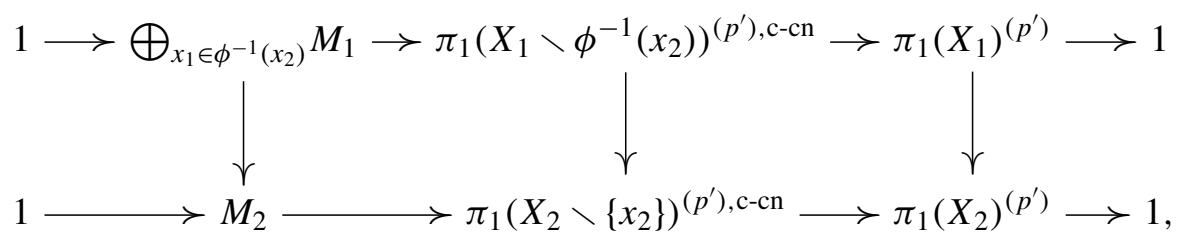

in which both rows are exact and the left vertical arrow is $\bigoplus_{x_{1} \in \phi^{-1}\left(x_{2}\right)} e_{x_{1}} \tau$, by the inertia-rigidity of $\sigma$. The commutativity of this last diagram implies that the image of the extension class of the top row (that is, $\left.\left(c_{1}\left(x_{1}\right)\right)_{x_{1} \in \phi^{-1}\left(x_{2}\right)}\right)$ under the map $H^{2}\left(\pi_{1}\left(X_{1}\right)^{\left(p^{\prime}\right)}, \bigoplus_{x_{1} \in \phi^{-1}\left(x_{2}\right)} M_{1}\right) \rightarrow H^{2}\left(\pi_{1}\left(X_{1}\right)^{\left(p^{\prime}\right)}, M_{1}\right)$ induced by $\bigoplus_{x_{1} \in \phi^{-1}\left(x_{2}\right)}\left[e_{x_{1}}\right]$ coincides with the image of the extension class of the bottom row (that is, $\left.c_{1}\left(x_{2}\right)\right)$ under the map $H^{2}\left(\pi_{1}\left(X_{2}\right)^{\left(p^{\prime}\right)}, M_{2}\right) \rightarrow H^{2}\left(\pi_{1}\left(X_{1}\right)^{\left(p^{\prime}\right)}, M_{1}\right)$ induced by $\sigma$ and $\tau^{-1}$. In other words, the image of $c_{1}\left(x_{2}\right)$ in $H^{2}\left(\pi_{1}\left(X_{1}\right)^{\left(p^{\prime}\right)}, M_{1}\right)$ 
coincides with $\sum_{x_{1} \in \phi^{-1}\left(x_{2}\right)} e_{x_{1}} c_{1}\left(x_{1}\right)$. From this follows the claim (in the special case), since the divisor $x_{2}$ maps to $\sum_{x_{1} \in \phi^{-1}\left(x_{2}\right)} e_{x_{1}} x_{1}$ via the above map $\operatorname{Div}_{X_{1}} \rightarrow$ $\operatorname{Div}_{X_{2}}$. Finally, consider the general case where $x_{2}$ may not be $k_{2}$-rational and each point of $\phi^{-1}\left(x_{2}\right)$ may not be $k_{1}$-rational. But this is reduced to the special case by considering suitable open subgroups of $\mathfrak{G}_{i}, i=1,2$, corresponding to constant field extensions $k_{i}^{\prime}$ of $k_{i}$. (Here, use the fact that the natural map $H^{2}\left(\pi_{1}\left(X_{i}\right)^{\left(p^{\prime}\right)}, M_{i}\right) \rightarrow H^{2}\left(\pi_{1}\left(X_{i} \times_{k_{i}} k_{i}^{\prime}\right)^{\left(p^{\prime}\right)}, M_{i}\right)$ is injective, which follows from the injectivity of the natural map $J_{X_{i}}\left(k_{i}\right) \rightarrow J_{X_{i}}\left(k_{i}^{\prime}\right)=J_{X_{i} \times k_{i}} k_{i}^{\prime}\left(k_{i}^{\prime}\right)$.) Thus, the claim follows.

Next, to treat the general case that we may possibly have $g_{2}=0$, consider any open subgroup $\mathfrak{H}_{2}$ of $\mathfrak{G}_{2}$ and set $\mathfrak{H}_{1} \stackrel{\text { def }}{=} \sigma^{-1}\left(\mathfrak{H}_{2}\right)$, which is an open subgroup of $\mathfrak{G}_{1}$. For each $i=1,2$, let $Y_{i}$ be the cover of $X_{i}$ corresponding to the open subgroup $\mathfrak{H}_{i} \subset \mathfrak{G}_{i}$, and $\ell_{i}$ the constant field of $Y_{i}$ (that is, the algebraic closure of $k_{i}$ in the function field of $Y_{i}$ ). Now, assume that the genus of $Y_{2}$ is positive. Then it follows from the preceding argument that the homomorphism $\operatorname{Div}_{Y_{1}} \rightarrow \operatorname{Div}_{Y_{2}}$ maps $D_{Y_{1}}$ into $D_{Y_{2}}$. In particular, by functoriality, the image of $D_{X_{1}}$ in $\operatorname{Div}_{X_{2}}$ is mapped into $D_{Y_{2}} \subset \operatorname{Div}_{Y_{2}}$ under the natural map $\operatorname{Div}_{X_{2}} \rightarrow \operatorname{Div}_{Y_{2}}$. Or, equivalently, the image of $D_{X_{1}}$ in $\operatorname{Div}_{X_{2}} / D_{X_{2}}$ lies in the kernel of $\operatorname{Div}_{X_{2}} / D_{X_{2}} \rightarrow \operatorname{Div}_{Y_{2}} / D_{Y_{2}}$. This last map is identified with the natural map

$$
\operatorname{Pic}_{X_{2}} /\left(J_{X_{2}}\left(k_{2}\right)\{p\}\right) \rightarrow \operatorname{Pic}_{Y_{2}} /\left(J_{Y_{2}}\left(\ell_{2}\right)\{p\}\right)
$$

induced by the pull-back of line bundles. Thus, by considering the norm map, we see that the kernel in question is killed by the degree $\left[\mathfrak{G}_{2}: \mathfrak{H}_{2}\right]$ of the cover $Y_{2} \rightarrow X_{2}$, and hence so is the image of $D_{X_{1}}$ in $\operatorname{Div}_{X_{2}} / D_{X_{2}}$.

Observe that the greatest common divisor of $\left[\mathfrak{G}_{2}: \mathfrak{H}_{2}\right]$, where $\mathfrak{H}_{2}$ runs over all open subgroups of $\mathfrak{G}_{2}$ such that the corresponding cover has positive genus, is 1 . (Indeed, if $g_{2}>0$, this is trivial, and, if $g_{2}=0$, this follows, for example, from Kummer theory.) Thus, the image of $D_{X_{1}}$ in $\operatorname{Div}_{X_{2}} / D_{X_{2}}$ must be trivial, as desired.

(ii) For $i=1,2$, let $\tilde{D}_{X_{i}}$ denote the inverse image of $D_{X_{i}} \subset \operatorname{Div}_{X_{i}}\left(\subset \widehat{\operatorname{Div}}_{X_{i}}\right)$ in $H^{1}\left(\mathfrak{G}_{i}, M_{i}\right)$. It follows from (i) and the commutativity of diagram (4.3) that the natural injective homomorphism $H^{1}\left(\mathfrak{G}_{2}, M_{2}\right) \hookrightarrow H^{1}\left(\mathfrak{G}_{1}, M_{1}\right)$ induces a natural injective homomorphism $\tilde{D}_{X_{2}} \hookrightarrow \tilde{D}_{X_{1}}$. Since $K_{i}^{\times}$is the inverse image of Pri $X_{X_{i}} \subset$ $\operatorname{Div}_{X_{i}}$ in $H^{1}\left(\mathfrak{G}_{i}, M_{i}\right)$ [Mochizuki 2007, Proposition 2.1(ii)], we have

$$
\tilde{D}_{X_{i}} / K_{i}^{\times} \stackrel{\sim}{\rightarrow} D_{X_{i}} / \operatorname{Pri}_{X_{i}} \stackrel{\sim}{\rightarrow} J_{X_{i}}\left(k_{i}\right)\{p\} .
$$

Thus, the injective homomorphism $\tilde{D}_{X_{2}} \hookrightarrow \tilde{D}_{X_{1}}$ induces $\left(K_{2}^{\times}\right)^{p^{n} \hookrightarrow} K_{1}^{\times}$, or, equivalently, $K_{2}^{\times} \hookrightarrow\left(K_{1}^{\times}\right)^{p^{-n}}$.

Since $\gamma\left(K_{2}^{\times}\right) /\left(\gamma\left(K_{2}^{\times}\right) \cap K_{1}^{\times}\right)$is injectively mapped into $\tilde{D}_{X_{1}} / K_{1}^{\times} \stackrel{\sim}{\rightarrow} J_{X_{1}}\left(k_{1}\right)\{p\}$, which is finite, $\gamma\left(K_{2}^{\times}\right) \cap K_{1}^{\times}$is of finite index in $\gamma\left(K_{2}^{\times}\right)$. Next, suppose that 
$\gamma\left(K_{2}^{\times}\right)=\gamma\left(K_{2}^{\times}\right) \cap\left(K_{1}^{\times}\right)^{p}$, or, equivalently, $\gamma\left(K_{2}^{\times}\right) \subset\left(K_{1}^{\times}\right)^{p}$. By the assumption that $a=0$, there exists an $x_{1} \in \Sigma_{X_{1}}$ such that $e_{x_{1}}=\mathfrak{e}_{x_{1}}^{\mathrm{t}}$. In particular, $e_{x_{1}}$ is prime to $p$. Set $x_{2} \stackrel{\text { def }}{=} \phi\left(x_{1}\right) \in \Sigma_{X_{2}}$ and take any $g \in K_{2}^{\times}$such that $\operatorname{ord}_{x_{2}}(g)=1$. Then, by the commutativity of diagram (4.3), we have $\operatorname{ord}_{x_{1}}(\gamma(g))=e_{x_{1}} \operatorname{ord}_{x_{2}}(g)=e_{x_{1}}$, which is prime to $p$. On the other hand, since $\gamma(g) \in\left(K_{1}^{\times}\right)^{p}, \operatorname{ord}_{x_{1}}(\gamma(g))$ must be divisible by $p$, which is absurd.

Finally, the desired commutativity of diagram follows easily from the functoriality of Kummer theory.

Next, let $x_{1} \in \Sigma_{X_{1}}$ and set $x_{2} \stackrel{\text { def }}{=} \phi\left(x_{1}\right) \in \Sigma_{X_{2}}$. Then (by choosing $\tilde{x}_{1} \in \Sigma_{\tilde{X}_{1}}$ above $x_{1}$ and $\tilde{x}_{2} \in \Sigma_{\tilde{X}_{2}}$ above $x_{2}$ such that $\left.\tilde{\phi}\left(\tilde{x}_{1}\right)=\tilde{x}_{2}\right)$ we have the natural commutative diagram

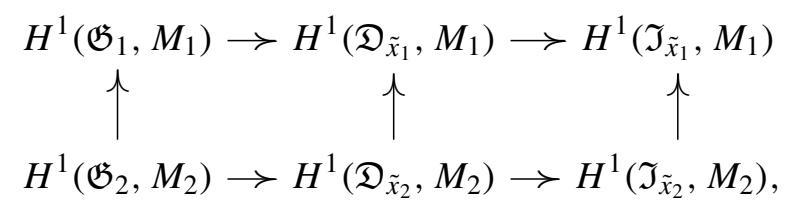

where the horizontal arrows are natural restriction maps and the vertical arrows are induced by $\left(\sigma, \tau^{-1}\right)$. By Kummer theory, this diagram can be identified with the natural commutative diagram

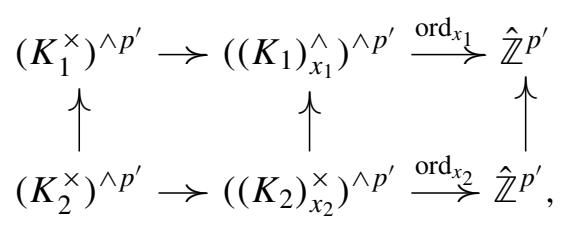

where the left horizontal arrows in the two rows arise from natural field homomorphisms $K_{1} \rightarrow\left(K_{1}\right)_{x_{1}}$ and $K_{2} \rightarrow\left(K_{2}\right)_{x_{2}}$ and the vertical arrows are induced by $\left(\sigma, \tau^{-1}\right)$. Further, the kernels of

$$
\left(\left(K_{1}\right)_{x_{1}}^{\times}\right)^{\wedge p^{\prime}} \stackrel{\operatorname{ord}_{x_{1}}}{\longrightarrow} \hat{\mathbb{Z}}^{p^{\prime}} \quad \text { and } \quad\left(\left(K_{2}\right)_{x_{2}}^{\times}\right)^{\wedge p^{\prime}} \stackrel{\operatorname{ord}_{x_{2}}}{\longrightarrow} \hat{\mathbb{Z}}^{p^{\prime}}
$$

are naturally identified with

$$
H^{1}\left(G_{k\left(x_{1}\right)}, M_{1}\right)=\left(k\left(x_{1}\right)^{\times}\right)^{\wedge p^{\prime}}=k\left(x_{1}\right)^{\times} \text {and } H^{1}\left(G_{k\left(x_{2}\right)}, M_{2}\right)=\left(k\left(x_{2}\right)^{\times}\right)^{\wedge p^{\prime}}=k\left(x_{2}\right)^{\times},
$$

respectively. Thus, in particular, the homomorphism $\left(\left(K_{2}\right)_{x_{2}}^{\times}\right)^{\wedge p^{\prime}} \rightarrow\left(\left(K_{1}\right)_{x_{1}}^{\times}\right)^{\wedge p^{\prime}}$ naturally induces a homomorphism $\iota_{x_{1}}: k\left(x_{2}\right)^{\times} \rightarrow k\left(x_{1}\right)^{\times}$that is identified with the homomorphism $H^{1}\left(G_{k\left(x_{2}\right)}, M_{2}\right) \rightarrow H^{1}\left(G_{k\left(x_{1}\right)}, M_{1}\right)$ induced by $\left(\sigma, \tau^{-1}\right)$. Here, the last homomorphism is injective by the fact $H^{0}\left(H_{k\left(x_{1}\right)}, M_{2}\right)=0$, where $H_{k\left(x_{1}\right)}$ stands for the (isomorphic) image of $G_{k\left(x_{1}\right)}$ in $G_{k\left(x_{2}\right)}$, which is open in $G_{k\left(x_{2}\right)}$.

We have two natural field homomorphisms $K_{1} \rightarrow K_{1}^{p^{-n}}$ : the first one is a natural embedding $i: K_{1} \hookrightarrow K_{1}^{p^{-n}}$ of degree $p^{n}$ and the second one is the isomorphism $j: K_{1} \stackrel{\sim}{\rightarrow} K_{1}^{p^{-n}}$ induced by the $p^{-n}$-th power map. According to these, we obtain 
two scheme morphisms $X_{1}^{p^{-n}} \rightarrow X_{1}$, where $X_{1}^{p^{-n}}$ stands for the integral closure of $X_{1}$ in $K_{1}^{p^{-n}}$. First, for closed points, these two morphisms give the same bijection

$$
\pi: \Sigma_{X_{1}^{p^{-n}}} \stackrel{\sim}{\rightarrow} \Sigma_{X_{1}}
$$

Let $x_{1} \in \Sigma_{X_{1}}$ and set $x_{1}^{p^{-n}} \stackrel{\text { def }}{=} \pi^{-1}\left(x_{1}\right)$. The two field homomorphisms $i$ and $j$ induce two isomorphisms $k\left(x_{1}\right) \rightarrow k\left(x_{1}^{p^{-n}}\right)$ of residue fields, which we shall denote by $\bar{i}\left(x_{1}\right)$ and $\bar{j}\left(x_{1}\right)$, respectively. Then we have $\bar{i}\left(x_{1}\right)=F^{n} \circ \bar{j}\left(x_{1}\right)$, where $F$ stands for the $p$ th power Frobenius map. Now, for valuations of functions, we have

$$
\operatorname{ord}_{x_{1}^{p^{-n}}} \circ i=p^{n} \operatorname{ord}_{x_{1}}, \quad \operatorname{ord}_{x_{1}^{p^{-n}}} \circ j=\operatorname{ord}_{x_{1}} .
$$

Finally, for values of functions, we have

$$
i(f)\left(x_{1}^{p^{-n}}\right)=\bar{i}\left(x_{1}\right)\left(f\left(x_{1}\right)\right), \quad j(f)\left(x_{1}^{p^{-n}}\right)=\bar{j}\left(x_{1}\right)\left(f\left(x_{1}\right)\right)
$$

for each $f \in K_{1}^{\times}$with $\operatorname{ord}_{x_{1}}(f) \geq 0$. Thus, in particular, $i(f)\left(x_{1}^{p^{-n}}\right)=j(f)\left(x_{1}^{p^{-n}}\right)^{p^{n}}$.

Lemma 4.14. Let $\gamma: K_{2}^{\times} \hookrightarrow\left(K_{1}^{\times}\right)^{p^{-n}}$ be the injective homomorphism in Lemma 4.13. Let $x_{1} \in \Sigma_{X_{1}}$ and set $x_{2} \stackrel{\text { def }}{=} \phi\left(x_{1}\right) \in \Sigma_{X_{2}}$. Then:

(i) For each $g \in K_{2}^{\times}$, we have

$$
\operatorname{ord}_{x_{1}^{p^{-n}}}(\gamma(g))=p^{n} e_{x_{1}} \operatorname{ord}_{x_{2}}(g) .
$$

(Namely, $\gamma$ is order-preserving with respect $\pi^{-1} \circ \phi$. See Definition 5.1.)

(ii) For each $g \in K_{2}^{\times}$with $\operatorname{ord}_{x_{2}}(g)=0$, we have $(\gamma(g))\left(x_{1}^{p^{-n}}\right)=i\left(x_{1}\right)\left(\iota_{x_{1}}\left(g\left(x_{2}\right)\right)\right)$. (Namely, $\gamma$ is value-preserving with respect $\pi^{-1} \circ \phi$ and

See Definition 5.2.)

$$
\left\{i\left(x_{1}\right) \circ \iota_{x_{1}}\right\}_{x_{1}^{p^{-n}} \in \Sigma_{X_{1}^{p^{-n}}}} .
$$

Proof. (i) and (ii) follow immediately from the commutativity of diagrams (4.3) and (4.4).

Fix a prime number $l \neq p$. For each $i=1,2$, let $k_{i}^{l}$ be the (unique) $\mathbb{Z}_{l^{-}}$ extension of $k_{i}$, set $K_{i}^{l} \stackrel{\text { def }}{=} K_{i} k_{i}^{l}$, and write $X_{i}^{l}$ for the normalization of $X_{i}$ in $K_{i}^{l}$. (Thus, $X_{i}^{l}=X_{i} \times_{k_{i}} k_{i}^{l}$.) Then the $p$-primary abelian subgroup $J_{X_{i}}\left(k_{i}^{l}\right)\{p\}$ of $J_{X_{i}}\left(k_{i}^{l}\right)$ is finite for $i=1$, 2. (See, for example, [Rosen 2002, Theorem 11.6] or [Saïdi and Tamagawa 2009, proof of Theorem 3.7].) So, write $p^{n_{0}}$ for the exponent of $J_{X_{1}}\left(k_{1}^{l}\right)\{p\}$. By passing to the limit over the finite extensions of $k_{i}$ contained in $k_{i}^{l}$ for $i=1,2$ (see Lemma 4.13(ii)), we get a natural embedding $\left(K_{2}^{l}\right)^{\times} \hookrightarrow\left(\left(K_{1}^{l}\right)^{\times}\right)^{p^{-n_{0}}}$. Now we apply a result from Section 5. (Observe that there are no vicious circles since the discussion of Section 5 does not depend on the contents of earlier sections.) More specifically, by Lemma 4.14 and Proposition 5.3, 
the embedding $\left(K_{2}^{l}\right)^{\times} \hookrightarrow\left(\left(K_{1}^{l}\right)^{\times}\right)^{p^{-n_{0}}}$ above arises from a (uniquely determined) embedding $K_{2}^{l} \hookrightarrow\left(K_{1}^{l}\right)^{p^{-n_{0}}}$ of fields. This embedding of fields restricts to the original embedding of multiplicative groups $K_{2}^{\times} \hookrightarrow\left(K_{1}^{\times}\right)^{p^{-n}}$. Thus, we conclude that this original embedding also arises from a (uniquely determined) embedding $K_{2} \hookrightarrow K_{1}^{p^{-n}}$ of fields.

Define the subfields $K_{2} \supset K_{2}^{\prime} \supset K_{2}^{\prime \prime}$ to be the inverse images of the subfields $K_{1}^{p^{-n}} \supset K_{1} \supset K_{1}^{p}$ in $K_{2}$. By Lemma 4.13(ii), there exists a finite subset $S \subset K_{2}$ such that $K_{2}=\bigcup_{\alpha \in S} K_{2}^{\prime} \alpha$. Since $K_{2}$ is an infinite field, this implies that $K_{2}^{\prime}$ is also an infinite field and that $K_{2}$ must be of dimension 1 as a $K_{2}^{\prime}$-vector space. Namely, $K_{2}=K_{2}^{\prime}$, or, equivalently, the above field homomorphism $K_{2} \hookrightarrow K_{1}^{p^{-n}}$ induces a field homomorphism $\gamma: K_{2} \hookrightarrow K_{1}$. Next, again by Lemma 4.13(ii), we have $\left[K_{2}^{\times}:\left(K_{2}^{\prime \prime}\right)^{\times}\right]>1$, that is, $K_{2} \supsetneq K_{2}^{\prime \prime}$. Equivalently, the field homomorphism $K_{2} \hookrightarrow K_{1}$ is separable.

Passing to the open subgroups $\mathfrak{H}_{1} \subset \mathfrak{G}_{1}, \mathfrak{H}_{2} \subset \mathfrak{G}_{2}$ with $\sigma\left(\mathfrak{H}_{1}\right) \subset \mathfrak{H}_{2}$ and applying the above arguments to $\mathfrak{H}_{1} \stackrel{\sigma}{\rightarrow} \mathfrak{H}_{2}$, we obtain naturally a (separable) field homomorphism $\tilde{\gamma}: \tilde{K}_{2} \rightarrow \tilde{K}_{1}$ which restricts to the above (separable) field homomorphism $K_{2} \rightarrow K_{1}$.

Lemma 4.15 (compatibility with the Galois action). Let $g_{1} \in \mathfrak{G}_{1}$, and let $g_{2} \stackrel{\text { def }}{=}$ $\sigma\left(g_{1}\right) \in \mathfrak{G}_{2}$. Then the following diagram is commutative:

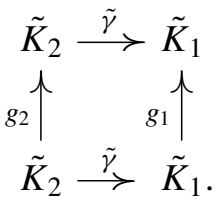

Proof. Let $\mathfrak{H}_{2} \subset \mathfrak{G}_{2}$ be an open normal subgroup and set

$$
\mathfrak{H}_{1} \stackrel{\text { def }}{=} \sigma^{-1}\left(\mathfrak{H}_{2}\right),
$$

which is an open normal subgroup of $\mathfrak{G}_{1}$. For $i=1,2$, let $F_{i} / K_{i}$ be the finite Galois subextension of $\tilde{K}_{i} / K_{i}$ corresponding to $\mathfrak{H}_{i} \subset \mathfrak{G}_{i}$, and denote by $Y_{i}$ the integral closure of $X_{i}$ in $F_{i}$. We have commutative diagrams

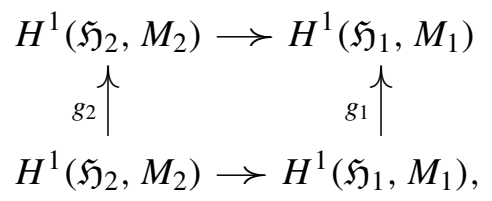

where $g_{i}: H^{1}\left(\mathfrak{H}_{i}, M_{i}\right) \rightarrow H^{1}\left(\mathfrak{H}_{i}, M_{i}\right)$ denotes the automorphism induced by the action of $g_{i}$ on $\mathfrak{H}_{i}$, and the horizontal maps are naturally induced by $\left(\sigma, \tau^{-1}\right)$ (see 
Lemma 4.11(i)), and

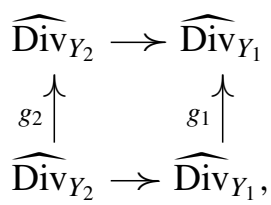

where the map $g_{i}: \widehat{\operatorname{Div}}_{Y_{i}} \rightarrow \widehat{\operatorname{Div}}_{Y_{i}}$ is the automorphism naturally induced by the action of $g_{i}$ on $Y_{i}$ (see Remark 4.2(iv)). Further, the above diagrams commute with each other, via the maps $H^{1}\left(\mathfrak{H}_{i}, M_{i}\right) \rightarrow \widehat{\operatorname{Div}}_{Y_{i}}$ in diagram (4.3) for $i=1,2$. Note that in the above diagrams the map $g_{i}: H^{1}\left(\mathfrak{H}_{i}, M_{i}\right) \rightarrow H^{1}\left(\mathfrak{H}_{i}, M_{i}\right)$ restricted to $F_{i}^{\times}$coincides with the automorphism $g_{i}: F_{i}^{\times} \rightarrow F_{i}^{\times}$. Therefore, we deduce this commutative diagram, from which the assertion follows:

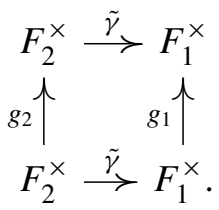

Finally, we shall prove the uniqueness of the field homomorphism $\tilde{\gamma}: \tilde{K}_{2} \rightarrow \tilde{K}_{1}$ that is Galois-compatible with respect to $\sigma$ and restricts to a separable homomorphism $K_{2} \rightarrow K_{1}$. In the profinite case, this uniqueness follows formally from the uniqueness in the assertion of the Isom-form proved in [Uchida 1977], as in the case of rigid homomorphisms in Section 3. (Observe that $\tilde{\gamma}: \tilde{K}_{2} \rightarrow \tilde{K}_{1}$ is then an isomorphism.) In general, however, we need some arguments which are not entirely formal, as follows.

So, let $\tilde{\gamma}^{\prime}: \tilde{K}_{2} \rightarrow \tilde{K}_{1}$ be another such field homomorphism. The field homomorphisms $\tilde{\gamma}$ and $\tilde{\gamma}^{\prime}$ induce field isomorphisms $\bar{k}_{2} \stackrel{\sim}{\rightarrow} \bar{k}_{1}$, say, $\bar{\gamma}$ and $\bar{\gamma}^{\prime}$, respectively, which are Galois-compatible with respect to $\sigma$. We may write $\bar{\gamma}^{\prime}=\varphi_{1}^{\alpha} \circ \bar{\gamma}$ for some $\alpha \in \hat{\mathbb{Z}}$, where $\varphi_{1} \in \operatorname{Gal}\left(\bar{k}_{1} / \mathbb{F}_{p}\right)$ stands for the $p$ th power Frobenius element. Further, the isomorphisms $\bar{\gamma}$ and $\bar{\gamma}^{\prime}$ induce $\hat{\mathbb{Z}}^{p^{\prime}}$-module isomorphisms $M_{2} \stackrel{\sim}{\rightarrow} M_{1}$, say, $\tau^{-1}$ and $\left(\tau^{\prime}\right)^{-1}$, respectively, which are Galois-compatible with respect to $\sigma$. Thus, we have $\left(\tau^{\prime}\right)^{-1}=\left[p^{\alpha}\right] \circ \tau^{-1}$. By Kummer theory, we have the commutative diagrams

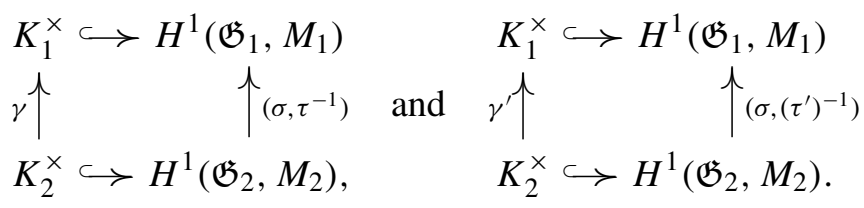

Thus, for each $g \in K_{2}^{\times}$, we have $\gamma^{\prime}(g)=\gamma(g)^{p^{\alpha}}$ in $\left(K_{1}^{\times}\right)^{\wedge p^{\prime}}$. Since both $\gamma$ and $\gamma^{\prime}$ are field homomorphisms, we deduce that $p^{\alpha} \in \mathbb{Q}_{>0}$, by taking a nonconstant function $g$ and considering valuations at suitable points. Thus, $\alpha \in \mathbb{Z}$, by [Chevalley 
1951, théorème 1]. Exchanging $\gamma$ and $\gamma^{\prime}$ if necessary, we may assume that $\alpha \geq 0$. Thus, $\gamma^{\prime}=F^{\alpha} \circ \gamma$, where $F$ stands for the $p$ th power Frobenius map. Since $\gamma^{\prime}$ is separable, we conclude $\alpha=0$, and hence $\gamma^{\prime}=\gamma$. Passing to the open subgroups $\mathfrak{H}_{1} \subset \mathfrak{G}_{1}, \mathfrak{H}_{2} \subset \mathfrak{G}_{2}$ with $\sigma\left(\mathfrak{H}_{1}\right) \subset \mathfrak{H}_{2}$, we conclude that $\tilde{\gamma}: \tilde{K}_{2} \rightarrow \tilde{K}_{1}$ is unique.

Thus, the proof of Theorem 4.8 is completed.

\section{Recovering the additive structure}

This section is devoted to the proof of Proposition 5.3, which was used in the proof of Theorem 4.8. We shall first axiomatize the set-up. We will use the following notations. For $i \in\{1,2\}$, let $X_{i}$ be a proper, smooth, geometrically connected curve over a field $k_{i}$ of characteristic $p_{i} \geq 0$. Let $K_{i}=K_{X_{i}}$ be the function field of $X_{i}$, and $\Sigma_{X_{i}}$ the set of closed points of $X_{i}$. Let

$$
\iota: K_{2}^{\times} \hookrightarrow K_{1}^{\times}
$$

be an embedding between multiplicative groups, which we extend to an embedding $\iota: K_{2} \hookrightarrow K_{1}$ between multiplicative monoids by setting $\iota(0)=0$. We assume that we are given a map

$$
\phi: \Sigma_{X_{1}} \rightarrow \Sigma_{X_{2}}
$$

that has finite fibers, that is, for any $x_{2} \in X_{2}$, the inverse image $\phi^{-1}\left(x_{2}\right) \subset \Sigma_{X_{1}}$ is a finite set.

Definition 5.1 (order-preserving maps). The map $\iota: K_{2} \rightarrow K_{1}$ is called orderpreserving with respect to the map $\phi$ if, for any $x_{2} \in \Sigma_{X_{2}}$ and any $x_{1} \in \phi^{-1}\left(x_{2}\right)$, there exists a positive integer $e_{x_{1} x_{2}}>0$ such that the following diagram commutes:

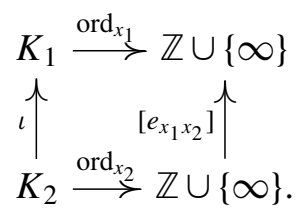

Here, $\left[e_{x_{1} x_{2}}\right]$ denotes the map of multiplication by $e_{x_{1} x_{2}}$ in $\mathbb{Z}$, which we extend naturally to $\mathbb{Z} \cup\{\infty\}$ by mapping $\infty$ to $\infty$.

Next, we assume that the map $\iota: K_{2} \rightarrow K_{1}$ is order-preserving with respect to the map $\phi: \Sigma_{X_{1}} \rightarrow \Sigma_{X_{2}}$. Further, we assume that we are given an embedding

$$
\iota_{x_{1} x_{2}}: k\left(x_{2}\right)^{\times} \hookrightarrow k\left(x_{1}\right)^{\times}
$$

between multiplicative groups for any $x_{2} \in \Sigma_{X_{2}}$ and any $x_{1} \in \phi^{-1}\left(x_{2}\right)$.

Definition 5.2 (value-preserving maps). The map $\iota: K_{2} \hookrightarrow K_{1}$ is called valuepreserving with respect to the map $\phi$ and the embeddings $\left\{\iota_{x_{1} x_{2}}\right\}_{\left(x_{1}, x_{2}\right)}$, where 
$\left(x_{1}, x_{2}\right)$ runs over all pairs of points $x_{2} \in \Sigma_{X_{2}}$ and $x_{1} \in \phi^{-1}\left(x_{2}\right)$ if, for any $f_{2} \in K_{2}^{\times}$ and any point $x_{2} \in \Sigma_{X_{2}}$ such that $x_{2} \cap \operatorname{Supp} \operatorname{div}\left(f_{2}\right)=\varnothing$, we have

$$
\iota_{x_{1}, x_{2}}\left(f_{2}\left(x_{2}\right)\right)=\iota\left(f_{2}\right)\left(x_{1}\right),
$$

where $f_{2}\left(x_{2}\right)$ denotes the value of $f_{2}$ at $x_{2}$ and $\iota\left(f_{2}\right)\left(x_{1}\right)$ the value of $\iota\left(f_{2}\right)$ at $x_{1}$.

If $\iota: K_{2} \hookrightarrow K_{1}$ is value-preserving, it particularly fits into the commutative diagram

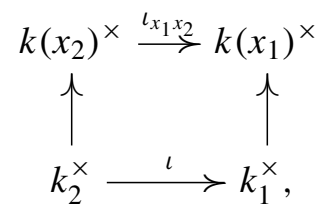

where the vertical maps are the natural embeddings. (Observe that $\iota$ maps $k_{2}$ into $k_{1}$, by the order-preserving assumption.)

Proposition 5.3 (recovering the additive structure). Assume that the embedding $\iota: K_{2} \hookrightarrow K_{1}$ is order-preserving with respect to the map $\phi$, and value-preserving with respect to the map $\phi$ and the embeddings $\left\{l_{x_{1} x_{2}}\right\}_{\left(x_{1}, x_{2}\right)}$, where the pair $\left(x_{1}, x_{2}\right)$ runs over all points $x_{2} \in \Sigma_{X_{2}}$ and $x_{1} \in \phi^{-1}\left(x_{2}\right)$. Assume further that $X_{2}\left(k_{2}\right)$ is an infinite set. Then the map $\iota$ is additive (and hence, a homomorphism of fields).

Proof. First, we shall prove that $\iota^{-1}\left(k_{1}\right)=k_{2}$. (Namely, $f \in K_{2}$ is constant if and only if $\iota(f) \in K_{1}$ is constant.) Indeed, set $F_{2} \stackrel{\text { def }}{=} \iota^{-1}\left(k_{1}\right)$. Note that $k_{i}^{\times}$coincides with the set of functions in $K_{i}^{\times}$with neither zeroes nor poles (or, equivalently, with no poles) anywhere in $\Sigma_{X_{i}}$. Now, by the order-preserving property of $\iota, F_{2} \backslash\{0\}$ coincides with the set of functions in $K_{2}^{\times}$with neither zeroes nor poles (or, equivalently, with no poles) in $\phi\left(\Sigma_{X_{1}}\right) \subset \Sigma_{X_{2}}$. It follows easily from this characterization that $F_{2}$ is a subfield of $K_{2}$ containing $k_{2}$. Since $K_{2}$ is a function field of one variable over $k_{2}$ and since $k_{2}$ is algebraically closed in $K_{2}$, we have either $F_{2}=k_{2}$ or that $F_{2}$ is also a function field of one variable over $k_{2}$. Suppose the latter, and let $W_{2}$ be the (proper, smooth, geometrically connected) curve over $k_{2}$ with function field $F_{2}$. Take any point $x_{1} \in \Sigma_{X_{1}}$ and let $w \in \Sigma_{W_{2}}$ be the image of $x_{1}$ under the composite map

$$
\Sigma_{X_{1}} \stackrel{\phi}{\rightarrow} \Sigma_{X_{2}} \rightarrow \Sigma_{W_{2}}
$$

where the second map arises from the cover $X_{2} \rightarrow W_{2}$ corresponding to the extension $L_{2} / F_{2}$. Now, by the Riemann-Roch theorem, there exists a function $f \in F_{2}$ having a pole at $w$. By the order-preserving property of $\iota$, the function $\iota(f) \in K_{1}$ must have a pole at $x_{1}$. This contradicts the definition of $F_{2}$. Therefore, we must have $F_{2}=k_{2}$, as desired.

We prove that $\phi: \Sigma_{X_{1}} \rightarrow \Sigma_{X_{2}}$ is surjective. Suppose otherwise and take $x_{2} \in$ $\Sigma_{X_{2}} \backslash \phi\left(\Sigma_{X_{1}}\right) \neq \varnothing$. By the Riemann-Roch theorem, there exists a nonconstant 
function $f \in K_{2}$ such that the pole divisor of $f$ is supported on $x_{2} \in \Sigma_{X_{2}}$. Then, by the order-preserving property of $\iota$, the function $\iota(f) \in K_{1}$ admits no poles, and hence $\iota(f) \in k_{1}$. As $\iota^{-1}\left(k_{1}\right)=k_{2}$, we thus have $f \in k_{2}$, which is absurd.

The rest of the proof is similar to the proof of [Saïdi and Tamagawa 2009, Proposition 4.4], where $\phi$ is a bijection. We first prove that $\iota$ restricted to $k_{2}$ is additive. Again by the Riemann-Roch theorem, there exists a nonconstant function $f \in K_{2}$ such that the pole $\operatorname{divisor} \operatorname{div}(f)_{\infty}$ of $f$ is supported on a unique point $x_{2} \in \Sigma_{X_{2}}: \operatorname{div}(f)_{\infty}=n x_{2}$, with $n>0$. For a nonzero constant $\alpha \in k_{2}$ we analyze the divisor of the function $\iota(f+\alpha)-\iota(f)$. We claim that

$$
\text { Supp } \operatorname{div}(\iota(f+\alpha)-\iota(f)) \subset \phi^{-1}\left(x_{2}\right) .
$$

Indeed, if $y_{1} \in \Sigma_{X_{1}}$ is such that $y_{2} \stackrel{\text { def }}{=} \phi\left(y_{1}\right) \neq x_{2}$, then $\operatorname{ord}_{y_{1}}(\iota(f+\alpha)) \geq 0$, and $\operatorname{ord}_{y_{1}}(\iota(f)) \geq 0$. Moreover, $\iota(f+\alpha)\left(y_{1}\right) \neq \iota(f)\left(y_{1}\right)$, as follows from the valuepreserving assumption, since $(f+\alpha)\left(y_{1}\right) \neq f\left(y_{1}\right)$. Thus,

$$
y_{1} \notin \operatorname{Supp} \operatorname{div}(\iota(f+\alpha)-\iota(f))
$$

and our claim follows. Further, if $x_{1} \in \phi^{-1}\left(x_{2}\right)$ is a pole of $\iota(f+\alpha)-\iota(f)$, we have $\left|\operatorname{ord}_{x_{1}}(\iota(f+\alpha)-\iota(f))\right| \leq n e_{x_{1} x_{2}}$. We deduce easily from this that there are only finitely many possibilities for the $\operatorname{divisor} \operatorname{div}(\iota(f+\alpha)-\iota(f))$. Since $k_{2}$ is infinite $\left(X_{2}\left(k_{2}\right)\right.$ being infinite), there exists an infinite subset $A \subset k_{2}^{\times}$such that $\operatorname{div}(\iota(f+\alpha)-\iota(f))$ is constant, for all $\alpha \in A$.

Let $\alpha \neq \beta$ be elements of $A$. Then $\operatorname{div}(\iota(f+\alpha)-\iota(f))=\operatorname{div}(\iota(f+\beta)-\iota(f))$, which implies

$$
\frac{\iota(f+\beta)-\iota(f)}{\iota(f+\alpha)-\iota(f)}=c \in k_{1}^{\times} .
$$

Observe that $\iota(f+\alpha)-\iota(f) \neq 0$, by the injectivity of $\iota$. Further, $c=\iota(\beta) / \iota(\alpha)$, as is easily seen by evaluating the function

$$
\frac{\iota(f+\beta)-\iota(f)}{\iota(f+\alpha)-\iota(f)}
$$

at a zero of the nonconstant function $\iota(f)$. Thus, we have $\iota(\beta)(\iota(f+\alpha)-\iota(f))=$ $\iota(\alpha)(\iota(f+\beta)-\iota(f))$, which is equivalent to

$$
\iota(f)(\iota(\alpha)-\iota(\beta))=\iota(\alpha) \iota(f+\beta)-\iota(\beta) \iota(f+\alpha) .
$$

Let

$$
g \stackrel{\text { def }}{=} \frac{\beta(f+\alpha)}{(\alpha-\beta) f}=\frac{\beta\left(1+\alpha f^{-1}\right)}{(\alpha-\beta)} .
$$

Note that $g$ is a nonconstant function, since $f$ is nonconstant. We have

$$
g+1=\frac{\beta(f+\alpha)}{(\alpha-\beta) f}+\frac{(\alpha-\beta) f}{(\alpha-\beta) f}=\frac{\beta \alpha+\alpha f}{\alpha f-\beta f}=\frac{\alpha(\beta+f)}{(\alpha-\beta) f} .
$$


Dividing this equality by $\iota(\alpha-\beta) \iota(f) \neq 0$, we obtain

$$
\frac{\iota(\alpha)-\iota(\beta)}{\iota(\alpha-\beta)}=\frac{\iota(\alpha) \iota(f+\beta)-\iota(\beta) \iota(\alpha+f)}{\iota(\alpha-\beta) \iota(f)} .
$$

Thus,

$$
\frac{\iota(\alpha)-\iota(\beta)}{\iota(\alpha-\beta)}=\frac{\iota(\alpha) \iota(f+\beta)}{\iota(\alpha-\beta) \iota(f)}-\frac{\iota(\beta) \iota(\alpha+f)}{\iota(\alpha-\beta) \iota(f)},
$$

which equals $\iota(g+1)-\iota(g)$. Further,

$$
\frac{\iota(\alpha)-\iota(\beta)}{\iota(\alpha-\beta)}=1,
$$

as follows by evaluating the function $\iota(g+1)-\iota(g)$ at a zero of the nonconstant function $\iota(g)$. Thus,

$$
\iota(g+1)=\iota(g)+1 .
$$

Take any $\zeta \in k_{2}$. Then, evaluating this equation at a zero of $\iota(g-\zeta)$, we obtain $\iota(\zeta+1)=\iota(\zeta)+1$. Now, for any $\xi, \eta \in k_{2}$, we have $\iota(\xi+\eta)=\iota(\xi)+\iota(\eta)$. Indeed, if $\eta=0$, this follows from $\iota(0)=0$. If $\eta \neq 0$, we have

$$
\iota(\xi+\eta)=\iota\left(\frac{\xi}{\eta}+1\right) \iota(\eta)=\left(\iota\left(\frac{\xi}{\eta}\right)+1\right) \iota(\eta)=\iota(\xi)+\iota(\eta) .
$$

Thus, $\left.\iota\right|_{k_{2}}$ is additive.

From this it follows easily that $\iota$ itself is additive. Indeed, let $h$ and $l$ be any elements of $K_{2}$, and let us prove $\iota(h+l)=\iota(h)+\iota(l)$. Take any $x_{2} \in X_{2}\left(k_{2}\right)$ which is neither a pole of $h$ nor a pole of $l$. Then, evaluating at any $x_{1} \in \phi^{-1}\left(x_{2}\right)$, we obtain

$$
\begin{aligned}
(\iota(h+l))\left(x_{1}\right) & =\iota_{x_{1} x_{2}}\left((h+l)\left(x_{2}\right)\right) \\
& =\iota_{x_{1} x_{2}}\left(h\left(x_{2}\right)+l\left(x_{2}\right)\right) \\
& =\iota\left(h\left(x_{2}\right)+l\left(x_{2}\right)\right) \\
& =\iota\left(h\left(x_{2}\right)\right)+\iota\left(l\left(x_{2}\right)\right) \\
& =\iota_{x_{1} x_{2}}\left(h\left(x_{2}\right)\right)+\iota_{x_{1} x_{2}}\left(l\left(x_{2}\right)\right) \\
& =(\iota(h))\left(x_{1}\right)+(\iota(l))\left(x_{1}\right) \\
& =(\iota(h)+\iota(l))\left(x_{1}\right),
\end{aligned}
$$

where the first and the sixth equalities follow from the value-preserving property, the second and the last equalities follow from the additivity of the evaluation maps, the third and the fifth equalities follow from the value-preserving property and the fact that $h\left(x_{2}\right), l\left(x_{2}\right) \in k_{2}$ (since $x_{2} \in X_{2}\left(k_{2}\right)$ ), and the fourth equality follows from the additivity of $\left.\iota\right|_{k_{2}}$. Now, since there are infinitely many such $x_{1}$ by assumption, the equality $\iota(h+l)=\iota(h)+\iota(l)$ must hold. Thus, Proposition 5.3 is proved. 


\section{Acknowledgement}

This work was done during a visit of Saïdi's to the Research Institute for Mathematical Sciences of Kyoto University, whose staff he would very much like to thank for their hospitality.

\section{References}

[Chevalley 1951] C. Chevalley, "Deux théorèmes d'arithmétique”, J. Math. Soc. Japan 3 (1951), 36-44. MR 13,440a Zbl 0044.03001

[Engler and Koenigsmann 1998] A. J. Engler and J. Koenigsmann, "Abelian subgroups of pro- $p$ Galois groups”, Trans. Amer. Math. Soc. 350:6 (1998), 2473-2485. MR 98h:12004 Zbl 0999.12004

[Engler and Nogueira 1994] A. J. Engler and J. B. Nogueira, "Maximal abelian normal subgroups of Galois pro-2-groups", J. Algebra 166:3 (1994), 481-505. MR 95h:12004 Zbl 0809.12004

[Fried and Jarden 1986] M. D. Fried and M. Jarden, Field arithmetic, Ergebnisse der Math. und ihrer Grenzgebiete (3) 11, Springer, Berlin, 1986. MR 89b:12010 Zbl 0625.12001

[Grothendieck and Raynaud 1971] A. Grothendieck and M. Raynaud, Revêtements étales et groupe fondamantal, edited by A. Grothendieck, Lecture Notes in Mathematics 224, Springer, Berlin, 1971. MR 50 \#7129 Zbl 0234.14002

[Harbater 1995] D. Harbater, "Fundamental groups and embedding problems in characteristic p", pp. 353-369 in Recent developments in the inverse Galois problem (Seattle, 1993), edited by M. D. Fried et al., Contemp. Math. 186, Amer. Math. Soc., Providence, RI, 1995. MR 97b:14035 Zbl 0858.14013

[Koenigsmann 2003] J. Koenigsmann, "Encoding valuations in absolute Galois groups", pp. 107132 in Valuation theory and its applications (Saskatoon, SK, 1999), vol. 2, edited by F.-V. Kuhlmann et al., Fields Institute Commun. 33, Amer. Math. Soc., Providence, 2003. MR 2004m:12012 Zbl 1050.12004

[Mochizuki 1999] S. Mochizuki, "The local pro- $p$ anabelian geometry of curves", Invent. Math. 138:2 (1999), 319-423. MR 2000j:14037 Zbl 0935.14019

[Mochizuki 2003] S. Mochizuki, "Topics surrounding the anabelian geometry of hyperbolic curves", pp. 119-165 in Galois groups and fundamental groups (Berkeley, 1999), edited by L. Schneps, Math. Sci. Res. Inst. Publ. 41, Cambridge Univ. Press, 2003. MR 2004m:14052 Zbl 1053.14029

[Mochizuki 2005] S. Mochizuki, "Galois sections in absolute anabelian geometry", Nagoya Math. J. 179 (2005), 17-45. MR 2006d:14022 Zbl 1129.14042

[Mochizuki 2007] S. Mochizuki, "Absolute anabelian cuspidalizations of proper hyperbolic curves", J. Math. Kyoto Univ. 47:3 (2007), 451-539. MR 2009d:14024 Zbl 1143.14305

[Neukirch 1969a] J. Neukirch, "Kennzeichnung der $p$-adischen und der endlichen algebraischen Zahlkörper”, Invent. Math. 6 (1969), 296-314. MR 39 \#5528 Zbl 0192.40102

[Neukirch 1969b] J. Neukirch, "Kennzeichnung der endlich-algebraischen Zahlkörper durch die Galoisgruppe der maximal auflösbaren Erweiterungen", J. Reine Angew. Math. 238 (1969), 135-147. MR 41 \#3450 Zbl 0201.05901

[Pop 1994] F. Pop, “On Grothendieck's conjecture of birational anabelian geometry", Ann. of Math.

(2) 139:1 (1994), 145-182. MR 94m:12007 Zbl 0814.14027

[Pop 1995] F. Pop, "Étale Galois covers of affine smooth curves. The geometric case of a conjecture of Shafarevich. On Abhyankar's conjecture", Invent. Math. 120:3 (1995), 555-578. MR 96k:14011 Zbl 0842.14017 
[Pop 2002] F. Pop, “The birational anabelian conjecture revisited”, preprint, 2002, available at http:// www.math.upenn.edu/ pop/Research/files-Res/finit-mf.pdf.

[Rosen 2002] M. Rosen, Number theory in function fields, Graduate Texts in Mathematics 210, Springer, New York, 2002. MR 2003d:11171 Zbl 1043.11079

[Saïdi and Tamagawa 2009] M. Saïdi and A. Tamagawa, "A prime-to- $p$ version of Grothendieck's anabelian conjecture for hyperbolic curves over finite fields of characteristic $p>0$ ", Publ. Res. Inst. Math. Sci. 45:1 (2009), 135-186. MR 2010j:14046 Zbl 1188.14016

[Serre 1967] J.-P. Serre, "Local class field theory", pp. 128-161 in Algebraic number theory: Proceedings of an instructional conference (Brighton, 1965), Academic Press, London, 1967. MR 36 \#3753

[Serre 1968] J.-P. Serre, Corps locaux, Hermann, Paris, 1968. MR 50 \#7096 Zbl 1095.11504

[Serre 1994] J.-P. Serre, Cohomologie galoisienne, 5th ed., Lecture Notes in Math. 5, Springer, Berlin, 1994. MR 96b:12010 Zbl 0812.12002

[Szamuely 2004] T. Szamuely, “Groupes de Galois de corps de type fini (d'après Pop)”, exposé 923, pp. 403-431 in Séminaire Bourbaki 2002/2003, Astérisque 294, Soc. math. de France, Paris, 2004. MR 2005k:12004 Zbl 1148.12300

[Tamagawa 1997] A. Tamagawa, "The Grothendieck conjecture for affine curves", Compositio Math. 109:2 (1997), 135-194. MR 99a:14035 Zbl 0899.14007

[Uchida 1976] K. Uchida, "Isomorphisms of Galois groups", J. Math. Soc. Japan 28:4 (1976), 617620. MR 55 \#5580 Zbl 0329.12013

[Uchida 1977] K. Uchida, "Isomorphisms of Galois groups of algebraic function fields", Ann. Math. (2) 106:3 (1977), 589-598. MR 57 \#273 Zbl 0372.12017

[Uchida 1981] K. Uchida, "Homomorphisms of Galois groups of solvably closed Galois extensions”, J. Math. Soc. Japan 33:4 (1981), 595-604. MR 83i:12011 Zbl 0464.12004

Communicated by Hélène Esnault

Received 2009-12-02 Revised 2010-05-01 Accepted 2010-06-06

M.Saidi@exeter.ac.uk University of Exeter, School of Engineering, Computing and Mathematics, Exeter EX44QF, United Kingdom

tamagawa@kurims.kyoto-u.ac.jp Kyoto University, Research Institute for Mathematical Sciences, Kyoto 606-8502, Japan 


\section{Algebra \& Number Theory}

www.jant.org

\section{EDITORS}

MANAGING EDITOR

Bjorn Poonen

Massachusetts Institute of Technology

Cambridge, USA

\author{
EDITORIAL BOARD CHAIR \\ David Eisenbud \\ University of California \\ Berkeley, USA
}

BOARD OF EDITORS

Georgia Benkart

Dave Benson

Richard E. Borcherds

John H. Coates

J-L. Colliot-Thélène

Brian D. Conrad

Hélène Esnault

Hubert Flenner

Edward Frenkel

Andrew Granville

Joseph Gubeladze

Ehud Hrushovski

Craig Huneke

Mikhail Kapranov

Yujiro Kawamata

János Kollár

Yuri Manin

Barry Mazur

Susan Montgomery
University of Wisconsin, Madison, USA

University of Aberdeen, Scotland

University of California, Berkeley, USA

University of Cambridge, UK

CNRS, Université Paris-Sud, France

University of Michigan, USA

Universität Duisburg-Essen, Germany

Ruhr-Universität, Germany

University of California, Berkeley, USA

Université de Montréal, Canada

San Francisco State University, USA

Hebrew University, Israel

University of Kansas, USA

Yale University, USA

University of Tokyo, Japan

Princeton University, USA

Northwestern University, USA

Harvard University, USA

University of Southern California, USA
Shigefumi Mori

Andrei Okounkov

Raman Parimala

Victor Reiner

Karl Rubin

Peter Sarnak

Michael Singer

Ronald Solomon

Vasudevan Srinivas

J. Toby Stafford

Bernd Sturmfels

Richard Taylor

Ravi Vakil

Michel van den Bergh

Marie-France Vignéras

Kei-Ichi Watanabe

Andrei Zelevinsky

Efim Zelmanov
RIMS, Kyoto University, Japan

Princeton University, USA

Emory University, USA

University of Minnesota, USA

University of California, Irvine, USA

Princeton University, USA

North Carolina State University, USA

Ohio State University, USA

Tata Inst. of Fund. Research, India

University of Michigan, USA

University of California, Berkeley, USA

Harvard University, USA

Stanford University, USA

Hasselt University, Belgium

Université Paris VII, France

Nihon University, Japan

Northeastern University, USA

University of California, San Diego, USA

\section{PRODUCTION}

contact@msp.org

Silvio Levy, Scientific Editor

Andrew Levy, Production Editor

See inside back cover or www.jant.org for submission instructions.

The subscription price for 2011 is US \$150/year for the electronic version, and \$210/year (+ \$35 shipping outside the US) for print and electronic. Subscriptions, requests for back issues from the last three years and changes of subscribers address should be sent to Mathematical Sciences Publishers, Department of Mathematics, University of California, Berkeley, CA 94720-3840, USA.

Algebra \& Number Theory (ISSN 1937-0652) at Mathematical Sciences Publishers, Department of Mathematics, University of California, Berkeley, CA 94720-3840 is published continuously online. Periodical rate postage paid at Berkeley, CA 94704, and additional mailing offices.

ANT peer review and production are managed by EditFLOw ${ }^{\mathrm{TM}}$ from Mathematical Sciences Publishers.

PUBLISHED BY

mathematical sciences publishers

http://msp.org/

A NON-PROFIT CORPORATION

Typeset in LATEX

Copyright $\odot 2011$ by Mathematical Sciences Publishers 


\section{Algebra \& Number Theory}

\section{Volume $5 \quad$ No. $2 \quad 2011$}

On the Hom-form of Grothendieck's birational anabelian conjecture in positive characteristic

\section{MOHAMED SAÏDI and AKIO TAMAGAWA}

Local positivity, multiplier ideals, and syzygies of abelian varieties

RoBERT LAZARSFELD, GIUSEPPE PARESCHI and MiHNEA POPA

KATHERINE STANGE

The basic geometry of Witt vectors, I The affine case

JAMES BORGER

Correction to a proof in the article Patching and admissibility over two-dimensional complete local domains

DANNY NEFTIN and ELAD PARAN 